\title{
Collagen fibre orientation in human bridging veins
}

\author{
Markos KAPELIOTIS ${ }^{a}$, Rebeca Alejandra GAVRILA LAIC ${ }^{a}$, Alvaro Jorge PEÑAS ${ }^{a}$, Jos \\ VANDER SLOTEN $^{\mathrm{a}}$, Pieter VANDEN BERGHE ${ }^{\mathrm{c}}$, Nele FAMAEY ${ }^{\mathrm{a}}$, Bart DEPREITERE ${ }^{\mathrm{b}}$ \\ ${ }^{a}$ Biomechanics Section, KU Leuven, Belgium \\ ${ }^{b}$ Department of Neurosurgery, University Hospital Gasthuisberg, KU Leuven, Belgium \\ ${ }^{c}$ Laboratory of Enteric Neuroscience (LENS), TARGID, KU Leuven, Leuven, Belgium
}

\begin{abstract}
Bridging veins (BV) drain the blood from the cerebral cortex into dural sinuses. BV have one end attached to the brain and the other to the superior sagittal sinus (SSS), which is attached to the skull. Relative movement between these two structures can cause BV to rupture producing acute subdural haematoma (ASDH), a head injury with a mortality rate between 30 and $90 \%$. A clear understanding of the BV's microstructure is required to increase the biofidelity of BV models when simulating head impacts.

Twelve fresh BV samples draining in the superior sagittal sinus (SSS) from a single human cadaver were cut open along their length and placed on an inverted multiphoton microscope. To ensure that the BV were aligned with the axial direction an in-house built, uniaxial tension set up was used. Two scans were performed per sample. Before the first scan, a minor displacement was applied to align the tissue, then a second scan was taken applying $50 \%$ strain. Each BV was scanned for a length of $5 \mathrm{~mm}$ starting from the drainage site into the SSS. Imaging was performed on a Zeiss LSM780 microscope with an 25x water immersion objective (NA 0.8), coupled to a tunable MaiTai DS (Spectraphysics) pulsed laser with the wavelength set at $850 \mathrm{~nm}$. Second harmonic and fluorescence signals were captured in forward and backward direction on binary GaAsP (BiG) detectors and stored as four colour Z-stacks. Prior to the calculation of the local orientations, acquired Z-stacks were denoised and enhanced to highlight fibrillar structures from the background. Then, for each Z-plane of the stack, the ImageJ plugin OrientationJ was used to extract the local $2 \mathrm{D}$ orientations of the fibres based on structure tensors.

Two kinds of collagen architectures were seen. The most common (8/12 samples) was single layered and had a uniform distribution of collagen. The less common (4/12 samples) had 2 layers and 7 to 34 times thicker collagen bundles on the outer layer. Fibre angle analysis showed that collagen was oriented mainly along the axial direction of the vessel. The von Mises fittings showed that in order to describe the fibre distribution 3 components were needed with mean angles $\mu$ at $-0.35,0.21,-0.02 \mathrm{rad}$ or $-20.2^{\circ}, 12.1^{\circ},-1.2^{\circ}$ relative to the vessel's axial direction which was also the horizontal scan direction.
\end{abstract}

Keywords: bridging veins, collagen fibres, orientation, multiphoton microscopy, acute subdural hematoma, traumatic brain injury

*Correspondence to: markos.kapeliotis@kuleuven.be, Celestijnenlaan 300 box 2419, 3001 Leuven, Belgium, tel: $+3216376068$ 


\section{Introduction}

Bridging veins (BV) are vessels that cross the subdural space and drain the blood from the cerebral cortex to the dural sinuses. In case of severe head impact, a relative motion between the brain and the skull can bring these vessels to rupture and produce acute subdural haematoma 40 (ASDH), an injury with a mortality rate ranging from 30-90\% 1 . Ruptures most often occur in BV draining into the superior sagittal sinus (SSS).

Despite the importance of the BV in the aetiology of $\mathrm{ASDH}^{[2]}$, still little is known about their histological, morphological and mechanical properties ${ }^{3}$. Understanding the BV's microstructure and, especially, their collagen fibre architecture is essential in order to accurately model the mechanical behaviour of the BV during head impacts.

The general structure of a venous wall consists of three layers:

- $\quad$ Tunica adventitia: external layer of the vein, composed of the connective tissue, that contains abundant collagen and elastin fibres with some elongated mesenchymal cells.

- $\quad$ Tunica media: intermediate layer, consisting of smooth muscle cells and elastin fibres.

- $\quad$ Tunica intima: inner layer, composed of multi-layered smooth endothelium covered by elastin tissue.

However, these three layers are not always visible in BV. 3 The distribution and orientation of the collagen fibres differ depending on the location along the BV 3,4 .

In the authors knowledge, the first study that investigated the microstructural characteristics of human BV was the one of Yamashima et al. 4]. They reported a loose webbing of fibres for the subdural part and a more dense structure for the subarachnoid part of the BV. More than half of the collagen fibre distribution was oriented in a circumferential manner, whereas the longitudinal fibres were much less abundant. Pang et al. used polarized microscopy of unstained fresh sections of porcine BV and showed that the principal direction of collagen fibres in the bridging veins was longitudinal apart from the outflow cuff region which is an area close to the connection to the SSS where circumferential distribution was seen 5 . Vignes et al. used transmission electron microscopy on human BV to demonstrate a helical orientation of the collagen fibres at the SSS-BV junction, in contrast to the linear orientation elsewhere ${ }^{6}$. Nierenberger et al., also on human BV, stated that even though fibre orientations are difficult to determine precisely, in the SSS the collagen fibres are mostly circumferentially oriented with a very homogenous structure composed of wavy collagen bundles with a volume fraction of approximately $61 \%$ and a bundle diameter of $10-20 \mu \mathrm{m} 7$.

Thin elastin fibres of approximately $0.3 \mu \mathrm{m}$ in diameter, uniformly distributed were also reported in this study. To summarize all findings, even though there are small differences within the studies, there is a general consensus about fibre orientation in different areas of the BV, but the fibre angles have not been determined precisely.

We hypothesized that collagen fibres tend to develop and form differently throughout BV length due to a difference in reinforcement and loading conditions between the SSS and the brain. The goal of this study is to be able to objectively assess the fibre distribution and the mean angle of collagen fibres throughout the length of the vessel, in order to better understand collagen fibre 

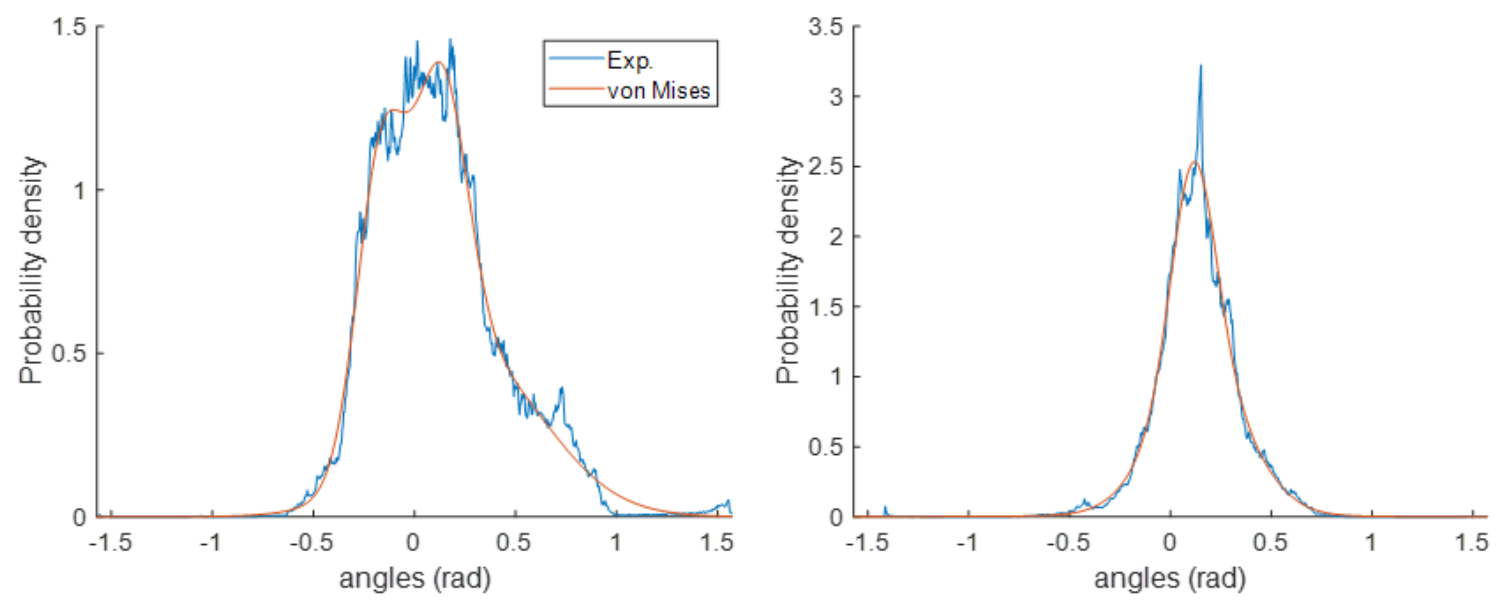

Figure 1: Fibre orientations with fitted von Mises mixtures for the same sample in an un-deformed (left) and deformed (right) state.

\section{Material and Methods}

\subsection{Source of material}

BV draining in the SSS were harvested from a single human cadaver (68 years old, female), at the Vesalius Institute of $\mathrm{KU}$ Leuven, and kept frozen at $-80^{\circ} \mathrm{C}$. The average number of the SSS draining BV in humans is 11 per hemisphere 8 . In this human specimen from a total of 17 samples, 12 were scanned. 6 BV samples originated from the frontal lobe, 3 from parietal lobe and 3 from the occipital lobe (Figure 4). The samples selected were the ones with bigger diameters and easier to scan. Also, some samples had to be discarded due to hardware failure. Each of the samples was left to thaw in a fridge at $4^{\circ} \mathrm{C}$ for $30 \mathrm{~min}$ before the experiment, which was sufficient time for the size and thickness of these veins. A dissection microscope and surgical tools were used to cut along the length of the vein in order to open the tubular structure into a flat tissue. To establish a reference coordinate system when scanning the veins, a tensile set up was designed and built to fit in the microscope. A part of the SSS was kept connected to the vein to use it as a clamping area when the tissue was mounted on the tension set up. Two dots were placed on the sides of each vein with Indian ink in order to measure width change. The width and length of the vessel were measured with a calliper and photos of the sample were taken before its mounting in the experimental device.

\subsection{Experimental set up}

This set up applies displacement through a micro screw that is operated manually. It is designed as a microscopy mounting plate in order to fit the stage of the inverted LSM 780 microscope and was 3D printed using Alumite (Figure 2) . The vessel's two ends were glued using acrylic glue on stainless steel blocks and were mounted on the uniaxial tension set up. The clamps had an alcove, that served as a glue container, where the samples were glued, as it can be seen in Figure $2 \mathrm{~B}$ that kept the glue from spreading to the tissue area that would be scanned or the coverglass. The steel blocks are made to rotate around the pins that connect them to the set up. The lumen of the 
SSS end of the vein was always clamped on the side where the micro screw was seen (Figure 2 . The BV was kept hydrated inside a bath with $0.9 \% \mathrm{NaCl}$ solution for the duration of the scan. Each scan lasted for approximately $1 \mathrm{hr}$ and 30 minutes. No force data were collected, as the objective of this set up was not to be used to derive material properties but rather to align the tissue in a way that gives a reference coordinate system to the data captured by the microscope.
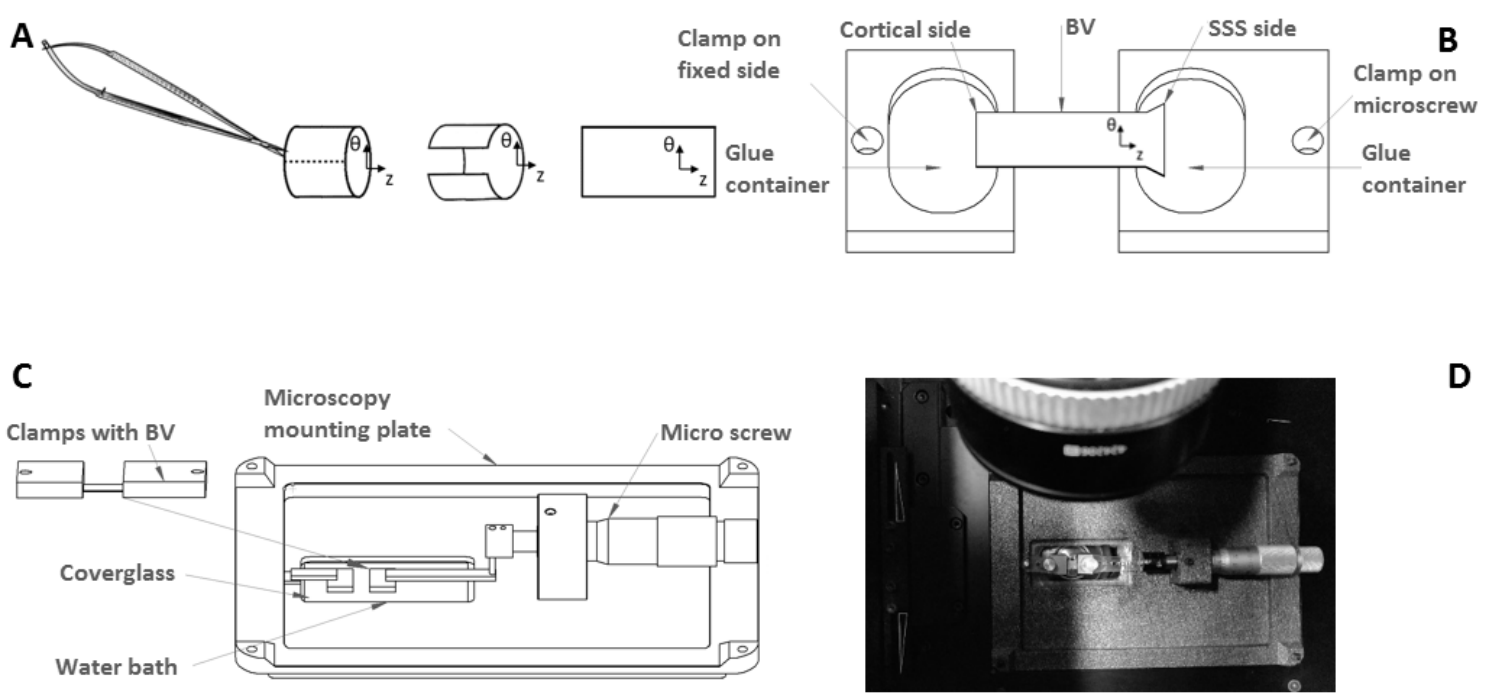

Figure 2: A: Under a dissection microscope the vein is opened along its axial direction. B: The BV is mounted on the clamps. C: The clamps are mounted on the tensile set up. D: The set up is then placed inside the microscope in order for the vein to be scanned.

\subsection{Imaging technique}

A Zeiss LSM 780 multiphoton microscope coupled to a tuneable MaiTai DS pulsed laser was used to generate images with a water immersion $25 \mathrm{x}$ objective lens (NA 0.8 ). The incoming wavelength was set at $850 \mathrm{~nm}$ in order to generate second harmonics (at $425 \mathrm{~nm}$ ) from collagen and elicit autofluorescence, the signals were captured in forward and backward direction on dual binary GaAsP $(\mathrm{BiG})$ detectors and stored as four channel 3D image stacks (forward channels in nm: 380-430 \& 465-515; backward: 420-480 \& 500-550). The scan had an average length of $4690 \mu \mathrm{m}$ in the axial direction and $1070 \mu \mathrm{m}$ in the circumferential direction of the vessel to investigate potential changes in the collagen architecture. Regarding the scanned width, i.e. the length in circumferential direction, sample 15 had a much bigger width than the rest at $4304 \mu \mathrm{m}$ and sample 16 had a much smaller width at $334 \mu \mathrm{m}$, the rest of the samples had a width ranging from 808 to $845 \mu \mathrm{m}$.

The 3D image stacks were stitched together using a Zeiss stitching module. The scanned area was carefully selected to be as remote as possible from the edges of the longitudinal cuts, in order to avoid bias in orientation due to the cutting process. Each sample was scanned twice, within a 4h range.

The scanned area's starting point was always as close as possible to the SSS connection point and spanned a maximum of $5 \mathrm{~mm}$ length. This limit was not related to the actual vein length but rather to practical issues, mainly scanning time and vessel length, i.e. sometimes the BV was not 
long enough to accommodate a scanning area of $5 \mathrm{~mm}$. Prior to the first scan a minor displacement to straighten the vein was applied and virtually no strain. This was also checked afterwards by measuring the width change of the vein before and after the straightening procedure. Before the second scan, a 50\% strain was applied to observe collagen fibre recruitment. The $50 \%$ strain magnitude was selected because it is the highest ultimate strain reported for BV 3 [ 9 and therefore the microstructural differences between these two loading states is of interest. The scanned area was also increased by $50 \%$ for the second scan to compare it to the first scan.

\subsection{Fibre orientation data analysis}

As it was mentioned above, the length of each of the BV scans had a maximum of $5 \mathrm{~mm}$, in order to investigate potential changes through the length of the vein each 3D image stack was analysed in its entirety and also was split in 4 blocks of equal size, see figure 3 . Each block was individually analysed in order to extract the changes of the collagen architecture along the BV length.

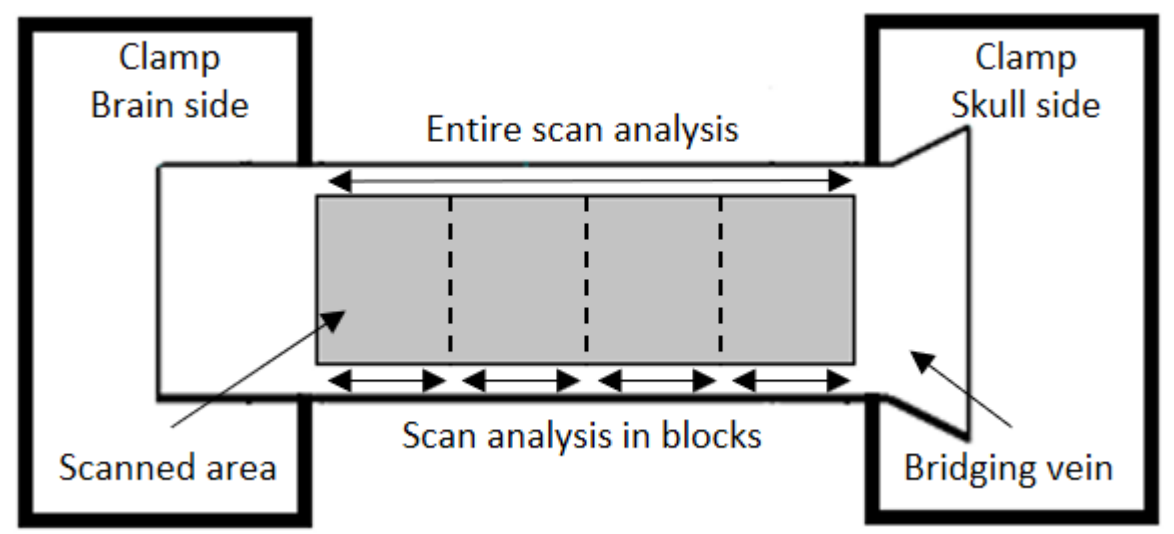

Figure 3: Graphical representation of the two approaches in analysing the scans, i.e. analysing each scan in its entirety and also analysing each scan after splitting it in 4 equally long blocks.

The 3D image stacks were enhanced by a three-step process consisting of a noise filtering step followed by a contrast enhancement and sharpening operations to highlight fibrillar structures from the background. Namely, raw images were processed by a penalized least squares-based denoising algorithm 10 . Then, the contrast of denoised images was enhanced by applying a gamma correction step followed by linear stretching operation on the grey scale values 11 . Finally, an unsharp masking filter was used to sharpen fibrillar structures 12. For each Z-plane the ImageJ plugin OrientationJ 13 was used to extract the local 2D orientations of the fibres based on structure tensors. In all samples a threshold of $15 \%$ in energy and coherency was applied using the OrientationJ plugin. Both energy and coherency are parameters ranging between $0 \%$ and $100 \%$, with $100 \%$ indicating highly oriented structures and $0 \%$ indicating isotropic areas, described in detail at the paper of Rezakhaniha and Agianniotis $\frac{14}{14}$. The resulting histograms show the orientation results for the full scanned area and the area split into 4 equally long blocks.

High bio-variability in BV has already been pointed out in literature 15 . To demonstrate what could be the preferred fibre direction across specimens, individual sample orientation measurements were summed up to create groups of samples. In this way, a mean fibre angle $(\mu)$ of the group can 
be seen along with its concentration of fibres $(\kappa)$ with respect to that angle and also the range limits of these angles, for both the un-deformed and deformed states. The differences along the length and between lobes were evaluated following this procedure.

A mixture of von Mises distributions was fit to each of the histograms to acquire the $\mu$, which is the mean angle, and the $\kappa$, which is a measure of concentration, of each component along with the component's percentage. The von Mises distribution is commonly used as a model for many circular data problems and is the n-periodic equivalent of a Gaussian distribution for directional statistics $\frac{16}{\text {. }}$. To fit the von Mises distribution mixture the mvmdist Matlab package was used. 1718 .

\section{Results}

The 12 BV samples were analysed with respect to their fibre orientation and their overall collagen architecture was assessed. Below the colour maps of the analysed BV samples are shown as well as the SSS and dura matter from where the samples were extracted in order to give a general overview (Figure 4).

\subsection{Collagen architecture}

Two different collagen architectures can be seen in our samples (Figure 5). The most common of the two, seen in 8 out of 12 samples, is single layered and is organized in evenly distributed bundles with a mean bundle size of $2.92 \mu \mathrm{m}$ and a standard deviation of $1.51 \mu \mathrm{m}$. The average diameter of these samples was $2.2 \mathrm{~mm}$ and the average wall thickness was $134 \mu \mathrm{m}$. On the other hand in 4 out of 12 samples, bigger bundles can be seen, having a double layered vessel wall and a mean width size of $48.58 \mu \mathrm{m}$ with a standard deviation of $22.50 \mu \mathrm{m}$. These samples had an average diameter of $3.7 \mathrm{~mm}$ and a wall thickness of $155 \mu \mathrm{m}, 2$ were located in the Frontal and 2 in the Parietal lobe.

\subsection{Fibre orientation results}

Each sample has been scanned in two states. First straightened but un-deformed and then under $50 \%$ strain. Since two different collagen architectures were observed, two representative cases, one of each, are shown below. The mean collagen orientations were analysed in two ways. First by using the whole image and second by splitting the image in 4 blocks of equal length, see figure 3 , to investigate potential orientation changes in collagen through the length.

In the main text two representative cases are shown, one having the single layered collagen architecture (sample 2) and one with the double layered collagen architecture (sample 6). For

the two representative cases, the local orientation colour maps of the collagen fibres of the two representative samples with and without deformation are shown, i.e. the hue-saturation-brightness (HSB) colour-coded maps showing the angles of the oriented structures in the image produced by OrientationJ, followed by their local orientation histograms, the von Mises distribution fittings and the von Mises parameters of each component below the colour maps of each sample (Figures 7 8, 9, 10, 11, 12, 14 . The component percentage is the percentage of each von Mises distribution in the total mixture, $\mu$ is the mean angle of each fibre family component, $\kappa$ is a measure of concentration with respect to the mean angle. The results from the analysis of each sample, apart from the representative cases are reported in the Appendix.

As it has been also mentioned in the materials and methods section, each scan from each sample is analyzed in two ways. First in each entirety, i.e. from one end till the other, and in four equal blocks, i.e. spliting the scan lengthwise in four pieces and analyzing each one individually as if it was a standalone scan(Figure 3 ). 


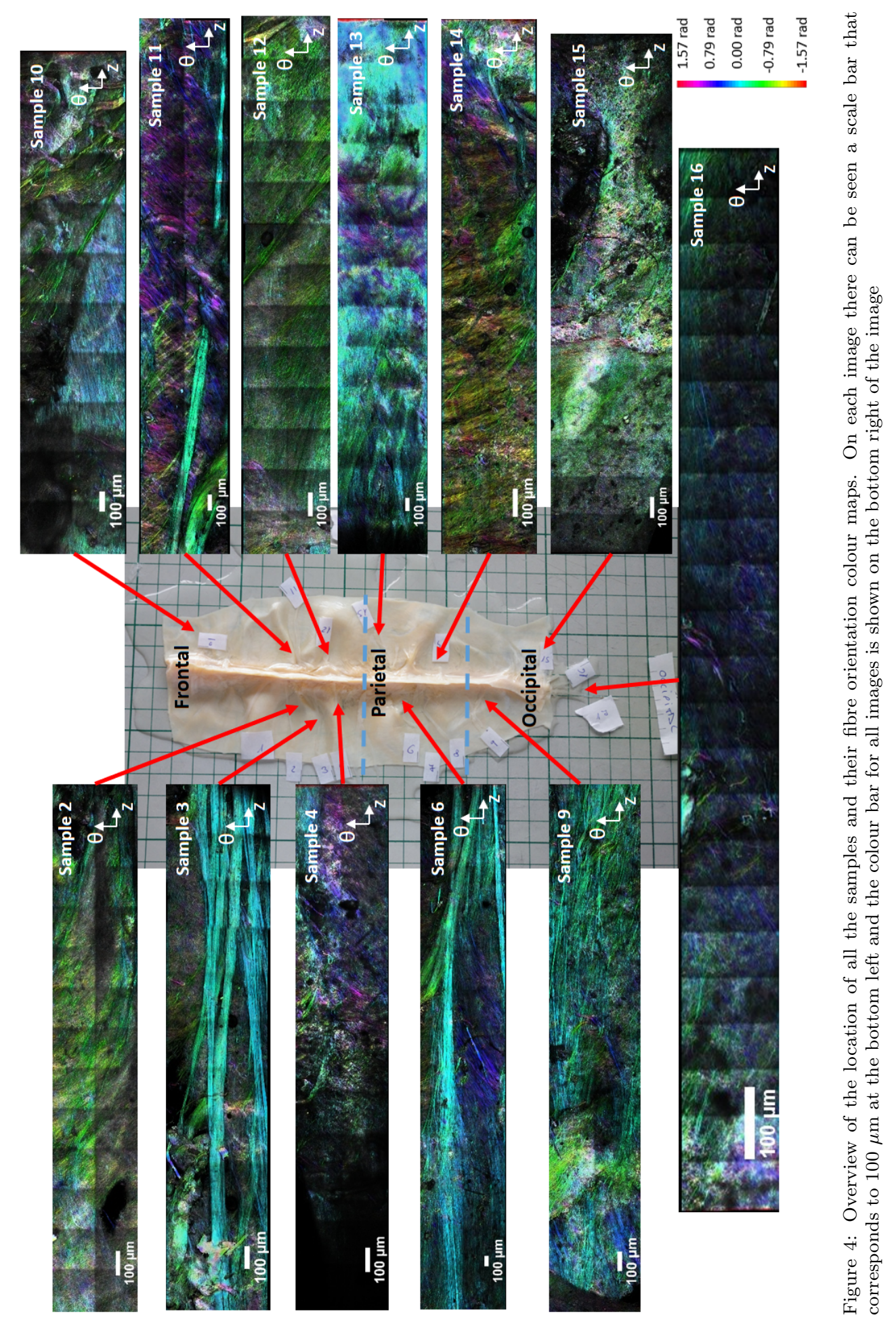



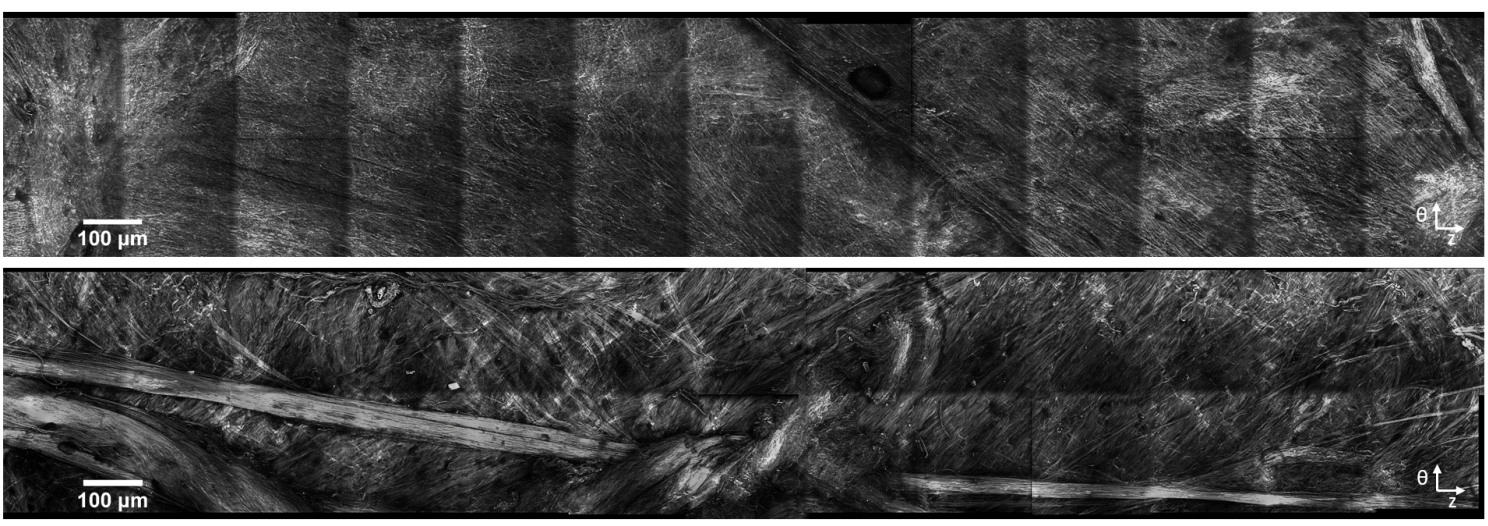

Figure 5: On the top a homogeneously structured BV, on the bottom a layered BV with much bigger collagen bundles. On the bottom right corner of both images the coordinate system is shown, with $\theta$ being the circumferential and $\mathrm{z}$ the axial direction.

Table 2: Summary table showing the sample name along with the brain region, the diameter size $(\varnothing)$, the wall thickness and a description of the microstructure

\begin{tabular}{|c|c|c|c|c|}
\hline$\overline{\text { Sample }}$ & Region & $\varnothing(\mathrm{mm})$ & THK $(\mu \mathrm{m})$ & Description \\
\hline 2 & $\mathrm{~F}, \mathrm{RH}$ & 1.3 & 129 & Single layer, homogeneously distributed with a mean bundle size of $2.43 \mu \mathrm{m}$ \\
\hline 3 & $\mathrm{~F}, \mathrm{RH}$ & 3.2 & 112 & 2 layers, first layer $44 \mu \mathrm{m}$ thick, second layer $68 \mu \mathrm{m}$ thick \\
\hline & & & & Big bundles mean size $52.39 \mu \mathrm{m}$ and small bundles mean size $1.65 \mu \mathrm{m}$ \\
\hline 4 & $\mathrm{~F}, \mathrm{RH}$ & 2.1 & 108 & Single layer, homogeneously distributed, with a mean bundle size of 2.59 \\
\hline 6 & $\mathrm{P}, \mathrm{RH}$ & 4.6 & 176 & $\begin{array}{l}2 \text { layers, first layer } 64 \mu \mathrm{m} \text { thick,second layer } 112 \mu \mathrm{m} \text { thick } \\
\text { Big bundles mean size } 45.54 \mu \mathrm{m} \text { and small bundles mean size } 4.11 \mu \mathrm{m}\end{array}$ \\
\hline 9 & $\mathrm{O}, \mathrm{RH}$ & 2.7 & 147 & Single layer, homogeneously distributed with a mean bundle size of $2.86 \mu \mathrm{m}$ \\
\hline 10 & $\mathrm{~F}, \mathrm{LH}$ & 3.8 & 153 & $\begin{array}{l}\text { Single layer, homogeneously distributed, with a mean bundle size of } 2.98 \mu \mathrm{m} \\
\text { with a mean bundle size of } 2.98 \mu \mathrm{m}\end{array}$ \\
\hline 11 & $\mathrm{~F}, \mathrm{LH}$ & 3.2 & 144 & $\begin{array}{l}2 \text { layers, first layer } 108 \mu \mathrm{m} \text { thick, second layer } 87 \mu \mathrm{m} \text { thick } \\
\text { Big bundles mean size } 55.66 \mu \mathrm{m} \text { and small bundles mean size } 1.79 \mu \mathrm{m}\end{array}$ \\
\hline 12 & $\mathrm{~F}, \mathrm{LH}$ & 2.1 & 114 & Single layer, homogeneously distributed, with a mean bundle size of $3.86 \mu \mathrm{m}$ \\
\hline 13 & $\mathrm{P}, \mathrm{LH}$ & 3.9 & 186 & $\begin{array}{l}2 \text { layers, first layer } 105 \mu \mathrm{m} \text { thick, second layer } 81 \mu \mathrm{m} \text { thick } \\
\text { Big bundles mean size } 40.74 \mu \mathrm{m} \text { and small bundles mean size } 1.65 \mu \mathrm{m}\end{array}$ \\
\hline 14 & $\mathrm{P}, \mathrm{LH}$ & 1.9 & 155 & Single layer, homogeneously distributed, with a mean bundle size of $2.61 \mu \mathrm{m}$ \\
\hline 15 & $\mathrm{O}, \mathrm{LH}$ & 2.6 & 156 & Single layer, homogeneously distributed, with a mean bundle size of $2.59 \mu \mathrm{m}$ \\
\hline 16 & $\mathrm{O}, \mathrm{LH}$ & 1.3 & 108 & Single layer, homogeneously distributed, with a mean bundle size of $5.9 \mu \mathrm{m}$ \\
\hline \multicolumn{5}{|c|}{$\begin{array}{l}\text { Ø: Diameter, F: Frontal lobe, P:Parietal lobe, O:Occipital lobe } \\
\text { RH: Right hemisphere, LH: Left hemisphere, THK: wall thickness }\end{array}$} \\
\hline
\end{tabular}




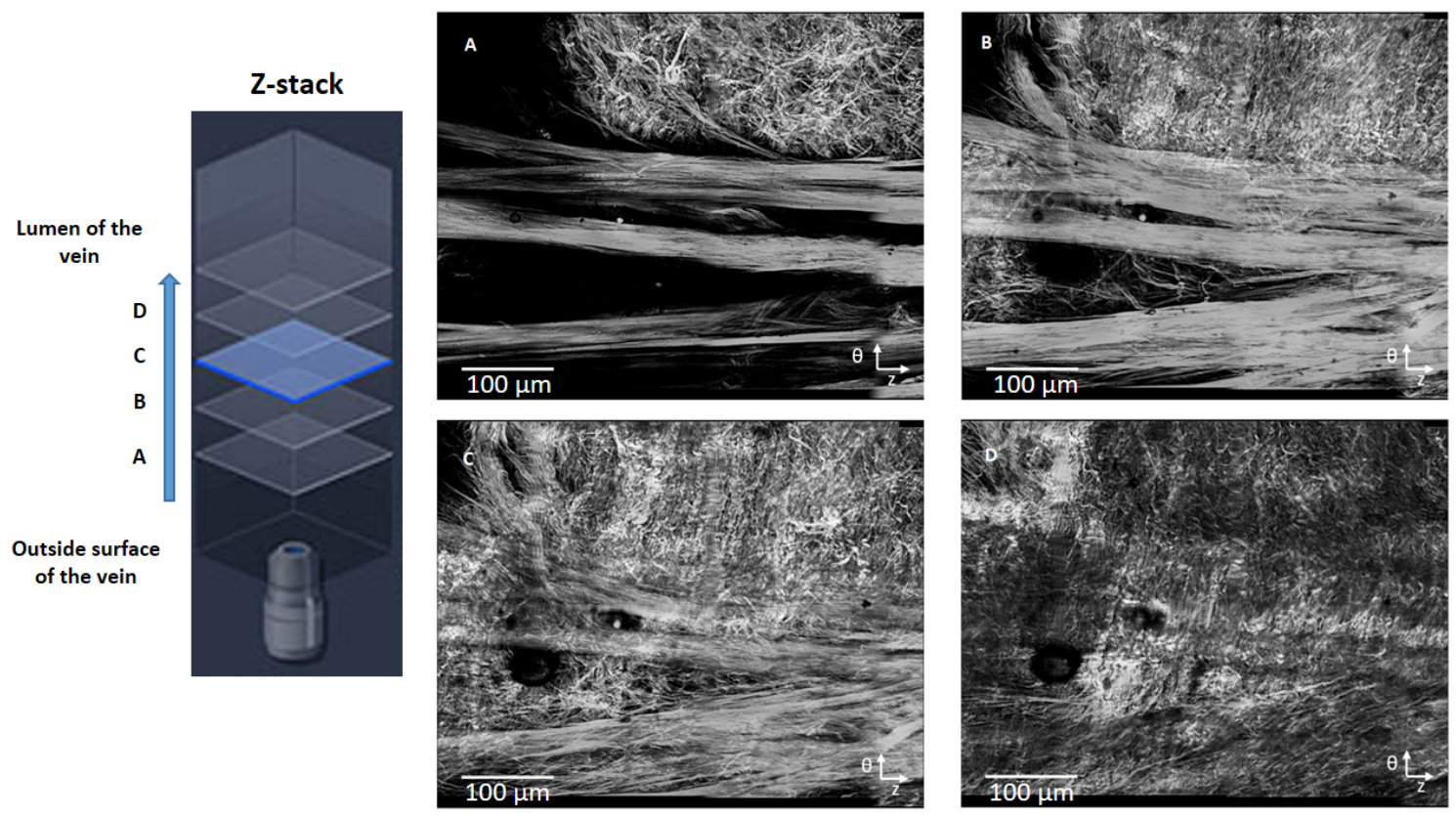

Figure 6: Starting from the outside surface of sample 3 (frontal right, $\varnothing 3.2 \mathrm{~mm}$, WT $112 \mu \mathrm{m}$ ), the transition between the two layers can be seen in sample 3 from $1 \mu \mathrm{m}$ in $\mathrm{A}$, to $24 \mu \mathrm{m}$ in B, to $44 \mu \mathrm{m}$ in C, to $68 \mu \mathrm{m}$ in D. $\theta$ is the circumferential and and $\mathrm{z}$ is the axial direction. 


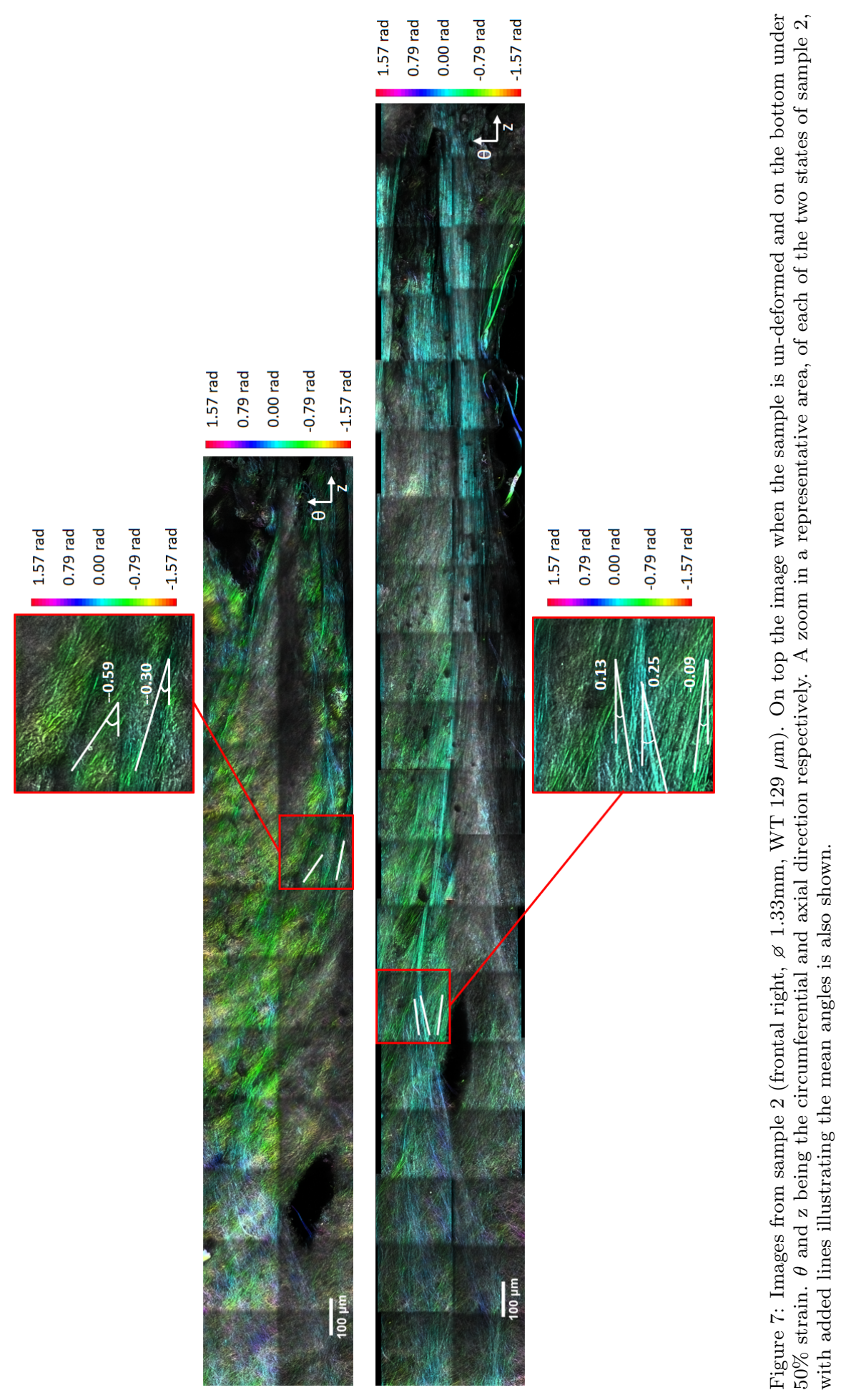




\subsection{Full length fibre orientation analysis-Sample 2}

Sample 2 has two fibre families at $-0.59 \mathrm{rad}\left(-34^{\circ}\right)$ and $-0.30 \mathrm{rad}\left(-17^{\circ}\right)$ with the first having a higher fibre concentration i.e $\kappa$ is 40 instead of 9.2 . When the same sample experiences $50 \%$ stretch then the fibre families mean angles $(\mu)$ move to $-0.09 \mathrm{rad}\left(-5.4^{\circ}\right)$ and $-0.13 \mathrm{rad}\left(7.2^{\circ}\right)$, i.e. closer to the axial direction and the fibre concentration $(\kappa)$ with respect to these angles also increases to 54 and 412.9 respectively, see figure 8 .

\begin{tabular}{|ccc|}
\hline C. P. (\%) & $\boldsymbol{\mu}$ (rad) & K \\
\hline 60.0 & -0.59 & 40.0 \\
\hline 40.0 & -0.30 & 9.2 \\
\hline & & \\
\hline
\end{tabular}

\begin{tabular}{|ccc|}
\hline C. P. (\%) & $\boldsymbol{\mu}$ (rad) & K \\
\hline 64.4 & -0.09 & 54.0 \\
\hline 23.4 & 1.26 & 412.9 \\
\hline 9.8 & 0.13 & 56.4 \\
\hline
\end{tabular}
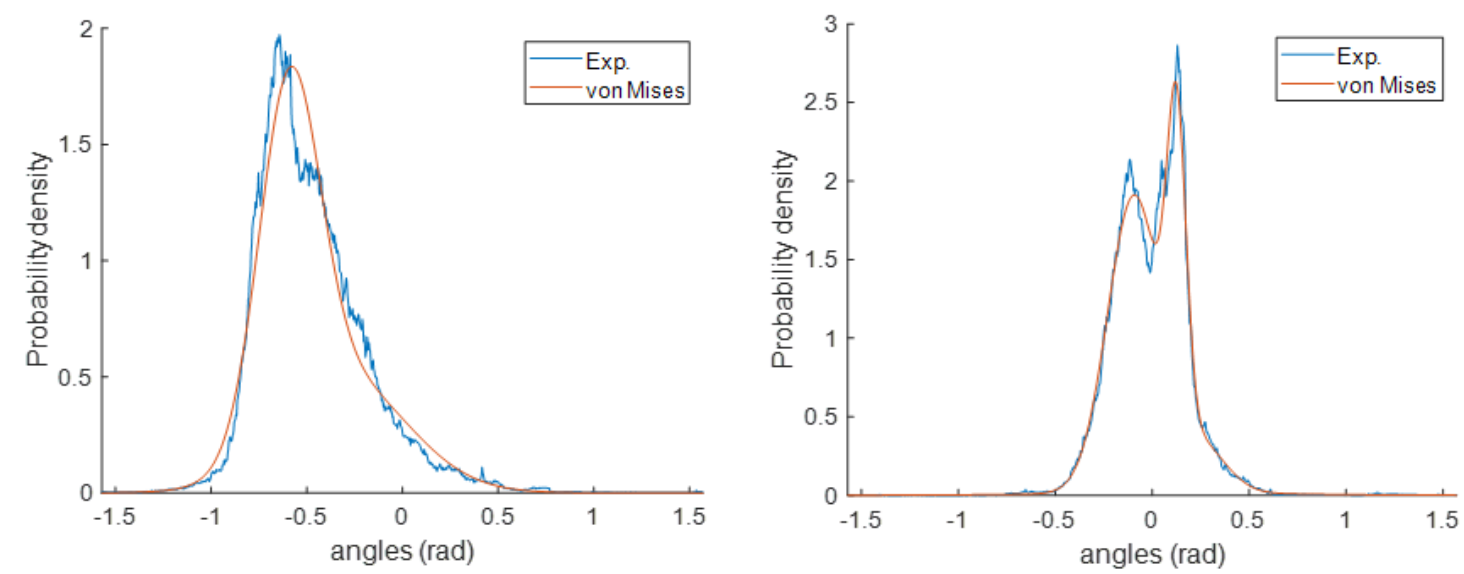

Figure 8: Collagen fibre orientation results for the entire length of the scan of sample 2, the left histogram being the initial scan and the right histogram being the second scan where $50 \%$ strain is applied. On top of the histograms the von Mises distribution fittings are plotted along with the von Mises parameters which are presented on the tables above each histogram.

\subsection{Block fibre orientation analysis-Sample 2}

When sample 2 is analysed in blocks two fibre families can be clearly identified on the end closer to the brain and when moving closer to the SSS the fibres appear concentrated into a single fibre family. That is also clear when seeing the component percentages going from almost $57 \%-43 \%$ on the brain side to $98 \%$ - $2 \%$ on the SSS side. When the $50 \%$ stretch is applied, the fibre families at both ends appear to get more concentrated and the mean angles $(\mu)$ move towards the axial direction while in the 2 middle blocks a second fibre family becomes more visible. Nonetheless, the fibres in the middle blocks are also more concentrated and oriented along the axial direction, see figure9.

\subsection{Full length fibre orientation analysis-Sample 6}

In the un-deformed state, two fibre families can be seen. The main one having a $\mu$ at $0.00 \mathrm{rad}$ $\left(0.1^{\circ}\right)$, with a $\kappa$ of 68 indicating high concentration with respect to that angle, and a minor one 

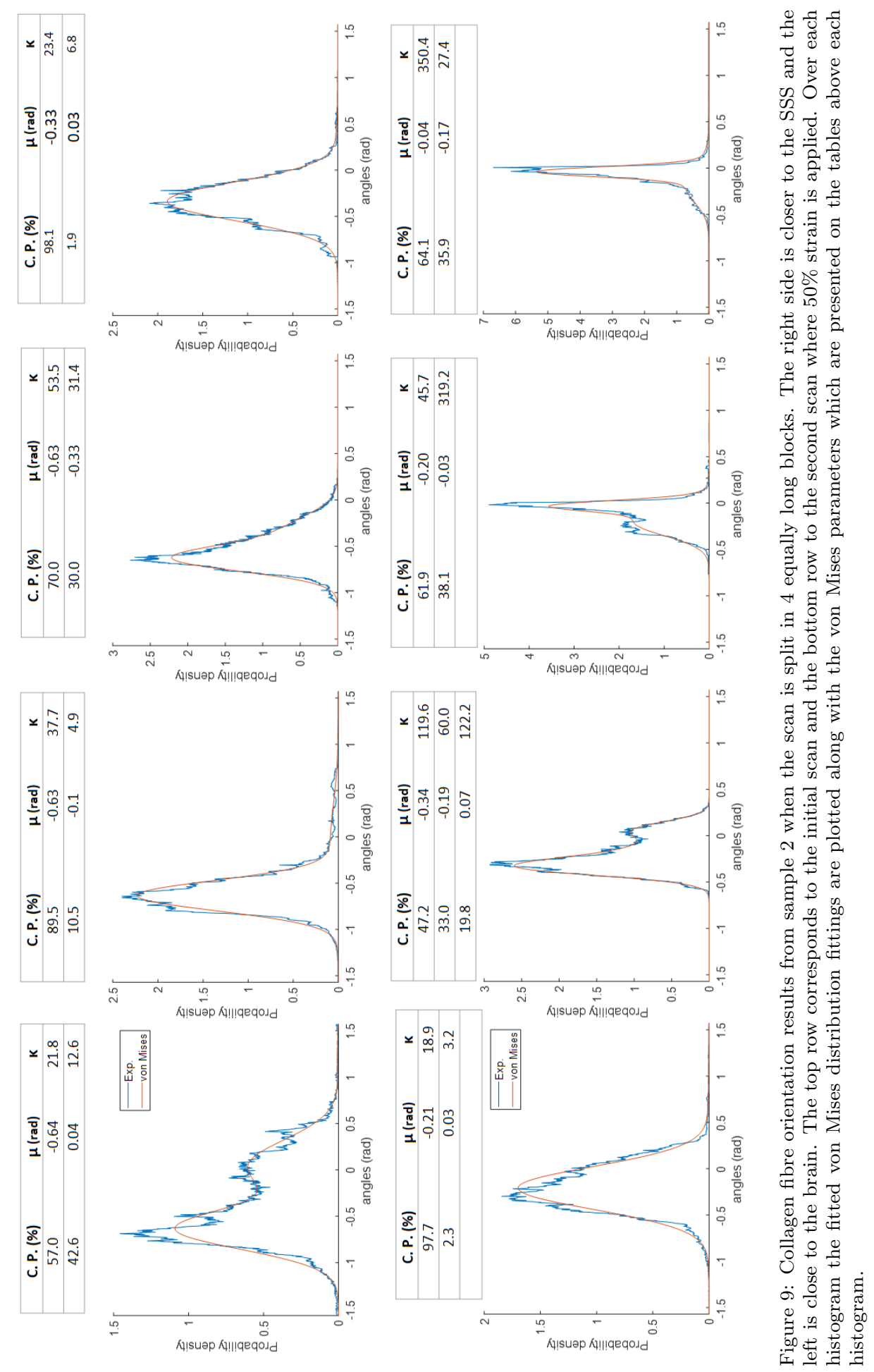


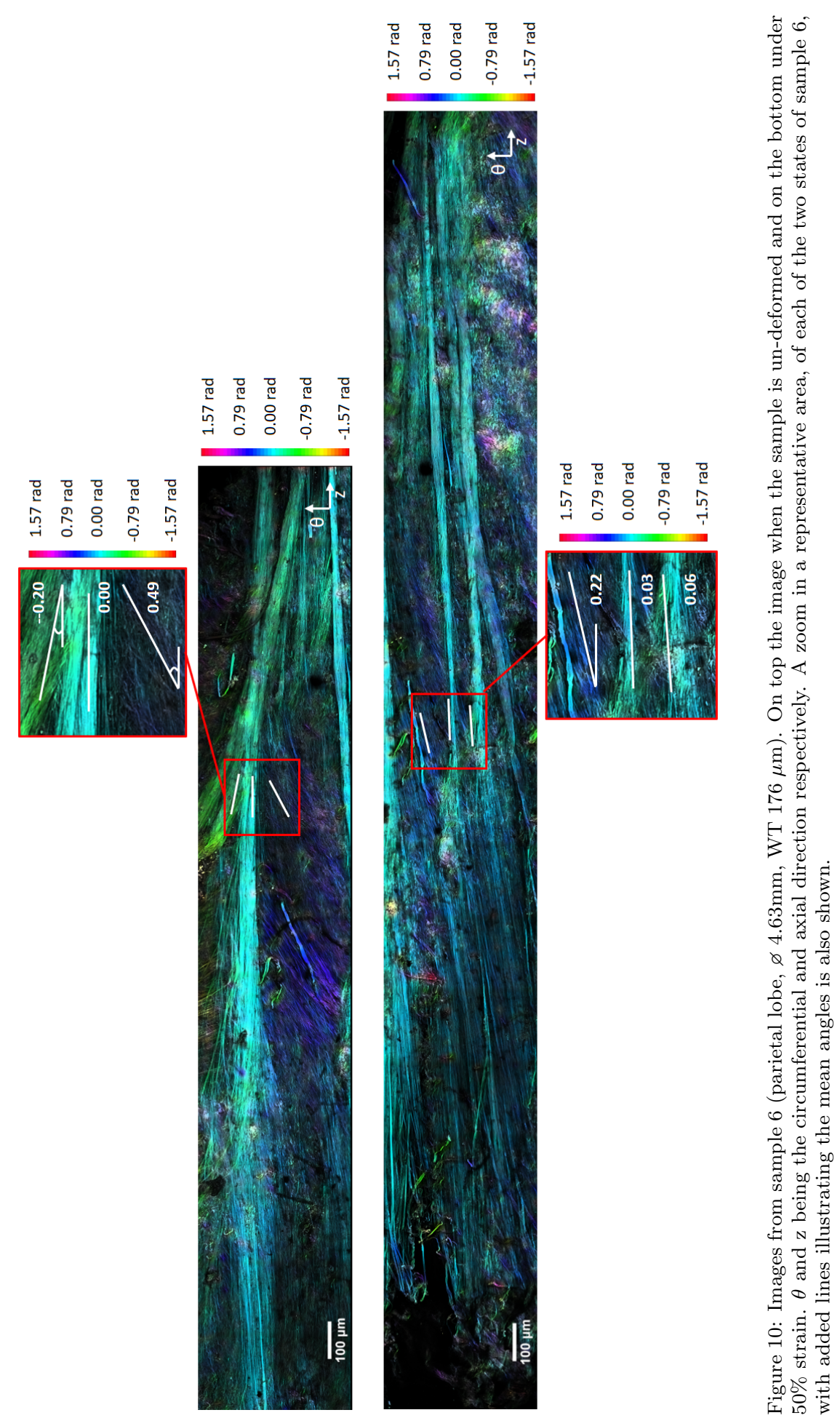


at $0.49 \mathrm{rad}\left(28^{\circ}\right)$. When the same sample goes under $50 \%$ stretch, two fibre families can be seen, the main family being in line with the axial direction as before but with even higher concentration $(\kappa=106.7)$ and the second being at $0.22 \mathrm{rad}\left(12.8^{\circ}\right)$ also with a high concentration $(\kappa=80.7)$, see figure11.

\begin{tabular}{|ccc|}
\hline C. P. (\%) & $\boldsymbol{\mu}$ (rad) & K \\
\hline 81.4 & 0.00 & 68.0 \\
\hline 9.4 & -0.20 & 9.8 \\
\hline 9.2 & 0.49 & 87.8 \\
\hline
\end{tabular}

\begin{tabular}{|ccc|}
\hline C. P. (\%) & $\boldsymbol{\mu}$ (rad) & K \\
\hline 57.1 & 0.03 & 106.7 \\
\hline 24.9 & 0.22 & 80.7 \\
\hline 17.9 & 0.06 & 8.7 \\
\hline
\end{tabular}
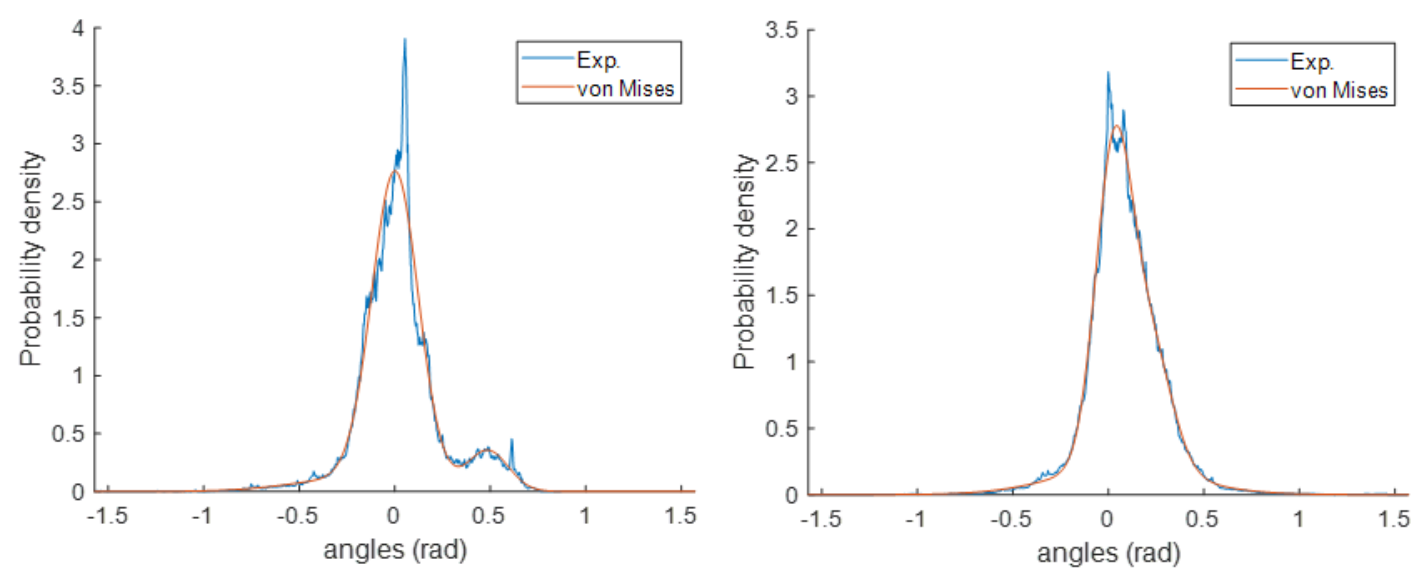

Figure 11: Collagen fibre orientation results for the entire length of the scan of sample 6, the left histogram being the initial scan and the right histogram being the second scan where $50 \%$ strain is applied. On top of the histograms the von Mises distribution fittings are plotted along with the von Mises parameters which are presented on the tables above each histogram.

\subsection{Block fibre orientation analysis-Sample 6}

When sample 6 is analysed in blocks one main fibre family oriented along the axial direction with high fibre concentration can be seen at the block on the brain side of the vein as well as the block on the SSS side of the vein. In the middle blocks on the other hand 2 to 3 fibre families can be seen. The middle and closer to the brain block has families with mean angles $(\mu)$ at $0.00 \mathrm{rad}$ $\left(0.0^{\circ}\right), 0.49 \operatorname{rad}\left(28.3^{\circ}\right)$ and $0.21 \mathrm{rad}\left(12^{\circ}\right)$ all with high concentration. The middle and closer to the SSS block has 2 families oriented close to each other and along the axial direction at $-0.11 \mathrm{rad}$ $\left(-6.1^{\circ}\right)$ and $-0.30 \mathrm{rad}\left(-2^{\circ}\right)$ and also one family with a mean angle at $0.44 \mathrm{rad}\left(25.2^{\circ}\right)$.

When under $50 \%$ stretch, in all 4 blocks a main fibre family oriented along the axial direction, being more concentrated than before, can be seen. Still, on the 2 blocks closer to the SSS an extra family with a mean angle of $0.23 \mathrm{rad}\left(13^{\circ}\right)$ and high fibre concentration can be detected having a $\kappa$ of 117,4 and 110.8 respectively, see figure 12

\subsection{Grouped sample fibre orientation results}

In order to portray general trends on fibre orientation across specimens, the orientation measurements from individual specimens were summed together to form different groups of samples. 


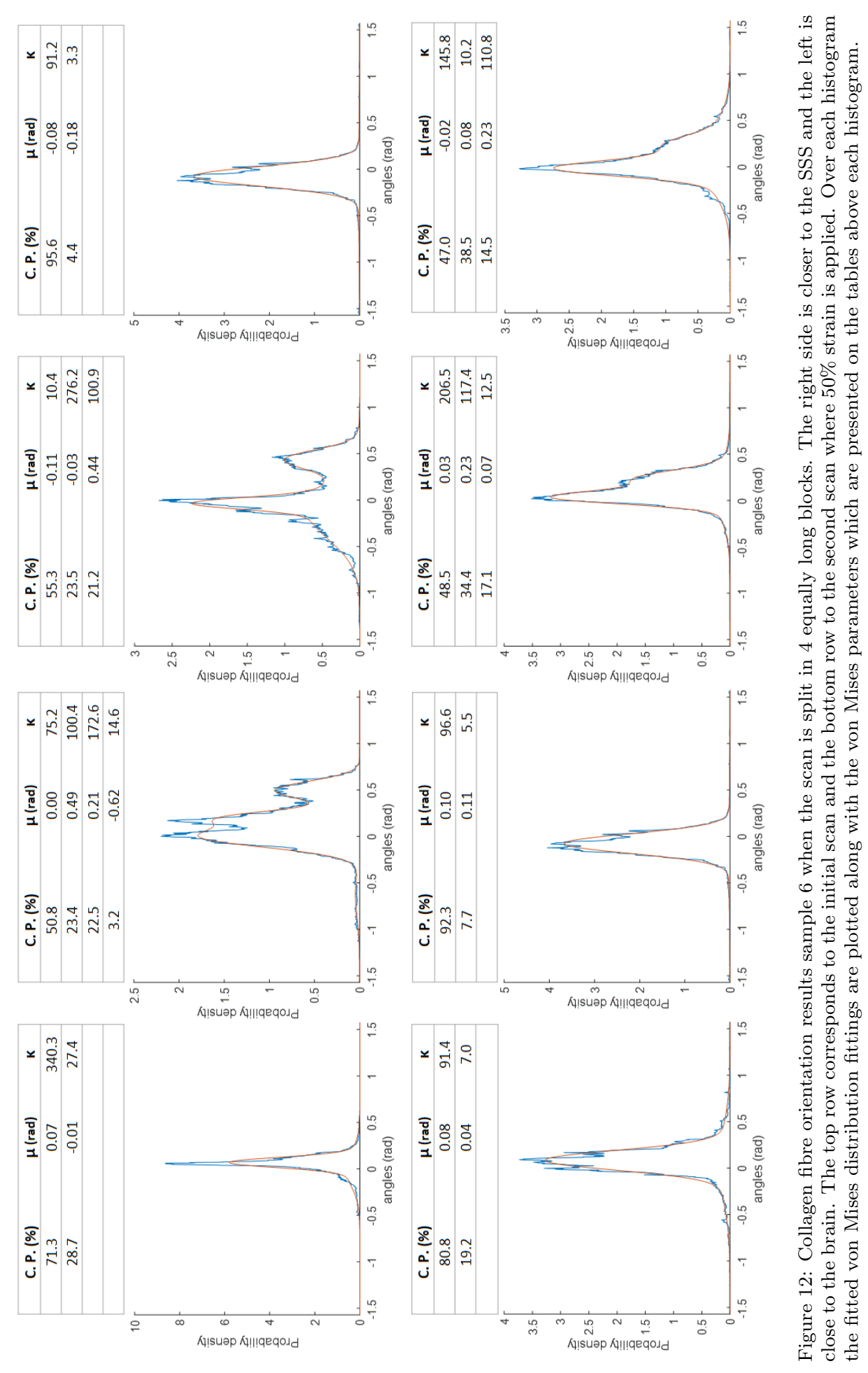


Four different groups were created, one for all the scanned samples and one group for the samples located in the frontal, parietal and occipital lobe respectively, see figure: 4. The fibre orientation histograms, the von Mises distribution fittings and the von Mises parameters of the group containing all samples is shown below. The groups containing the samples per lobe are located in the appendix.

\subsection{Full length fibre orientation analysis- all samples group}

The histograms from the group containing all measurements from all the samples (figure:(4) 14) is shown below, i.e. all the measurements from all the scans performed in the $0 \%$ strain state have been grouped together to create one histogram and then on that histogram a mixture of von Mises distributions has been fitted to provide von Mises parameters that can describe the whole group, the same has been done for the scans when the BV were under $50 \%$ strain.

Looking into that group we see that the most pronounced fibre angle is the one that is aligned with the axial direction. To obtain a good fit on the experimental data a mixture of 3 von Mises distributions was fitted on the combined data. The resulting angles of $-0.35,0.21,-0.02 \mathrm{rad}\left(-20^{\circ}\right.$, $\left.12^{\circ},-1^{\circ}\right)$ are also very close to the axial direction and the fibre family with the $\mu$ of $-0.02 \mathrm{rad}\left(-1^{\circ}\right)$ has also a high concentration with a $\kappa$ of 104.2.

The $50 \%$ strain all samples group showed, as it was expected, an increased recruitment of fibres along the axial direction. In deformed state the group of all the samples can be represented again by a mixture of 3 von Mises distributions with mean angles at $-0.12,0.08,0.02 \mathrm{rad}\left(-7.1^{\circ}, 4.3^{\circ}\right.$ ,$\left.-1.3^{\circ}\right)$ all being very close to the axial direction and with the last one having high concentration with a $\kappa=275.6$,see figure14.
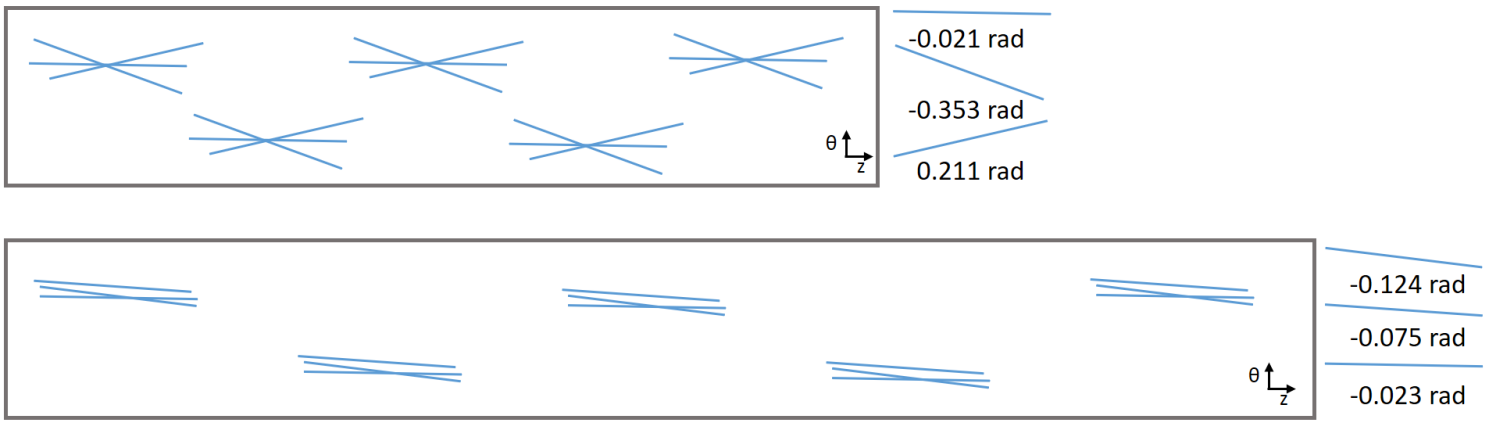

Figure 13: Graphical illustration of the fibre orientation of collagen for the combined measurements of all the samples.

\subsection{Block fibre orientation analysis - all samples group}

In the same way as in the previous subsection, all the measurements from all the scans performed in the $0 \%$ strain state have been grouped together to create a histogram per block group and then on the four resulting histograms, mixtures of von Mises distributions have been fitted to provide von Mises parameters per block group and the same procedure was also applied for the scans in the $50 \%$ strain state.

When analysed in blocks, in the un-deformed state, a mixture of 3 to 4 von Mises distributions is needed to represent the data. From the brain to the SSS there is always a main component with an angle close to the axial direction i.e. from -13.3 to 8.9. There are also mean angles far from the 


\begin{tabular}{|ccc|}
\hline C. P. (\%) & $\boldsymbol{\mu}$ (rad) & K \\
\hline 50.1 & -0.35 & 16.4 \\
\hline 37.0 & 0.21 & 7.3 \\
\hline 12.8 & -0.02 & 104.2 \\
\hline
\end{tabular}

\begin{tabular}{|ccc|}
\hline C. P. (\%) & $\boldsymbol{\mu}$ (rad) & K \\
\hline 73.0 & -0.12 & 12.8 \\
\hline 16.0 & -0.08 & 4.0 \\
\hline 10.9 & -0.02 & 275.6 \\
\hline
\end{tabular}
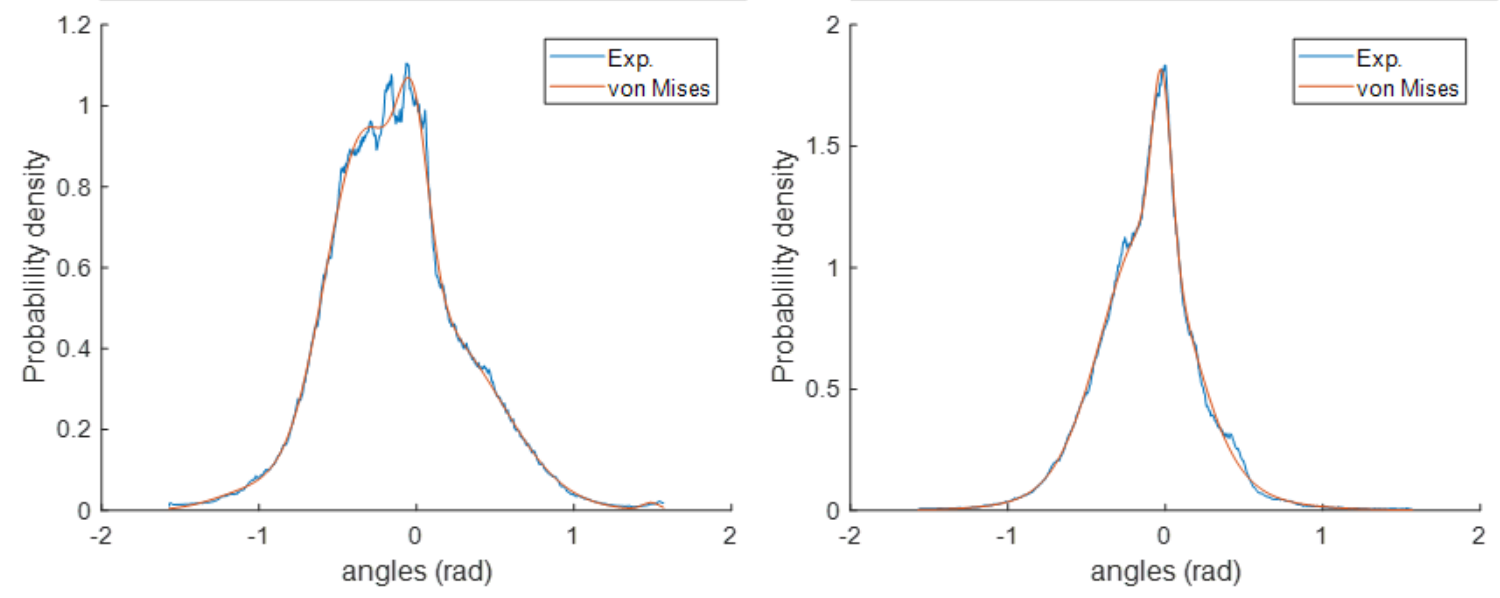

Figure 14: Collagen fibre orientation results for the group containing the scans for all three lobes and for the entire length. On top of the histograms the von Mises distribution fittings are plotted along with the von Mises parameters which are presented on the tables above each histogram.

axial direction with values between -54.9 to 38.1 . In the deformed state the group in all blocks is more concentrated with respect to the axial direction with mean angles values ranging from -19.6 to 4.7 , see figure15. In both un-deformed and deformed state the fibre orientation are more aligned and concentrated along the axial direction in the SSS block.

\section{Discussion}

In this study 12 fresh BV from a human cadaver were put under a multiphoton microscope in order to investigate their collagenous architecture and mainly their collagen fibre orientation. In previous studies on the microstructural properties of BV only qualitative data were given regarding the collagen fibre angles 4 6 7 5 3. In this study, the aim was to produce quantitative data to be used to improve fittings in BV mechanical testing i.e. by reducing the range of values that the fibre angle parameter can take in fittings of mechanical tests on structural material models such as the $\mathrm{GOH}$ material model as well as to improve the behaviour of the material models in FE simulations.

Other studies have used similar techniques to determine the collagen fibre distribution of venous tissue. Vesely et al. characterized the collagen fibre orientation of the media and adventitia layers of the abdominal vena cava. They reported the fibre orientations to be circumferential for the media layer and axial for the adventitia layer 19 . Nierenberger et al. characterized of the collagenous structure of porcine jugular vein walls and found two fibre families at $1.17 \mathrm{rad}\left(67^{\circ}\right)$ and $-1.32 \mathrm{rad}$ $\left(-76^{\circ}\right)$. When these veins were loaded in the axial direction the fibres reached $0.37 \mathrm{rad}\left(21^{\circ}\right)$ and $-0.23 \mathrm{rad}\left(-13^{\circ}\right)$ degrees ${ }^{20}$, i.e. the fibres reoriented towards the axial direction in a similar way as in our study. 

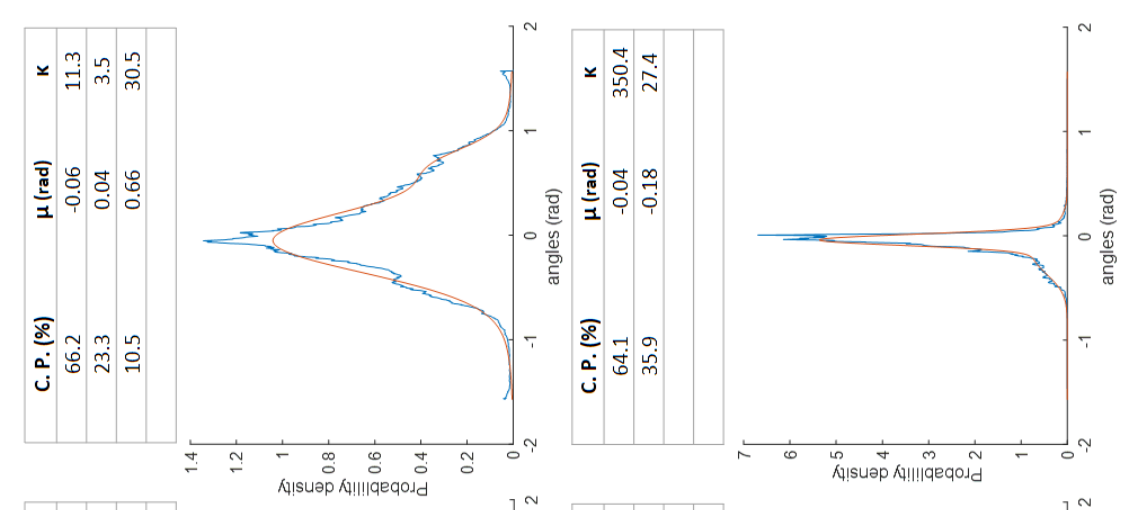

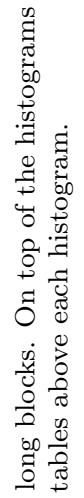
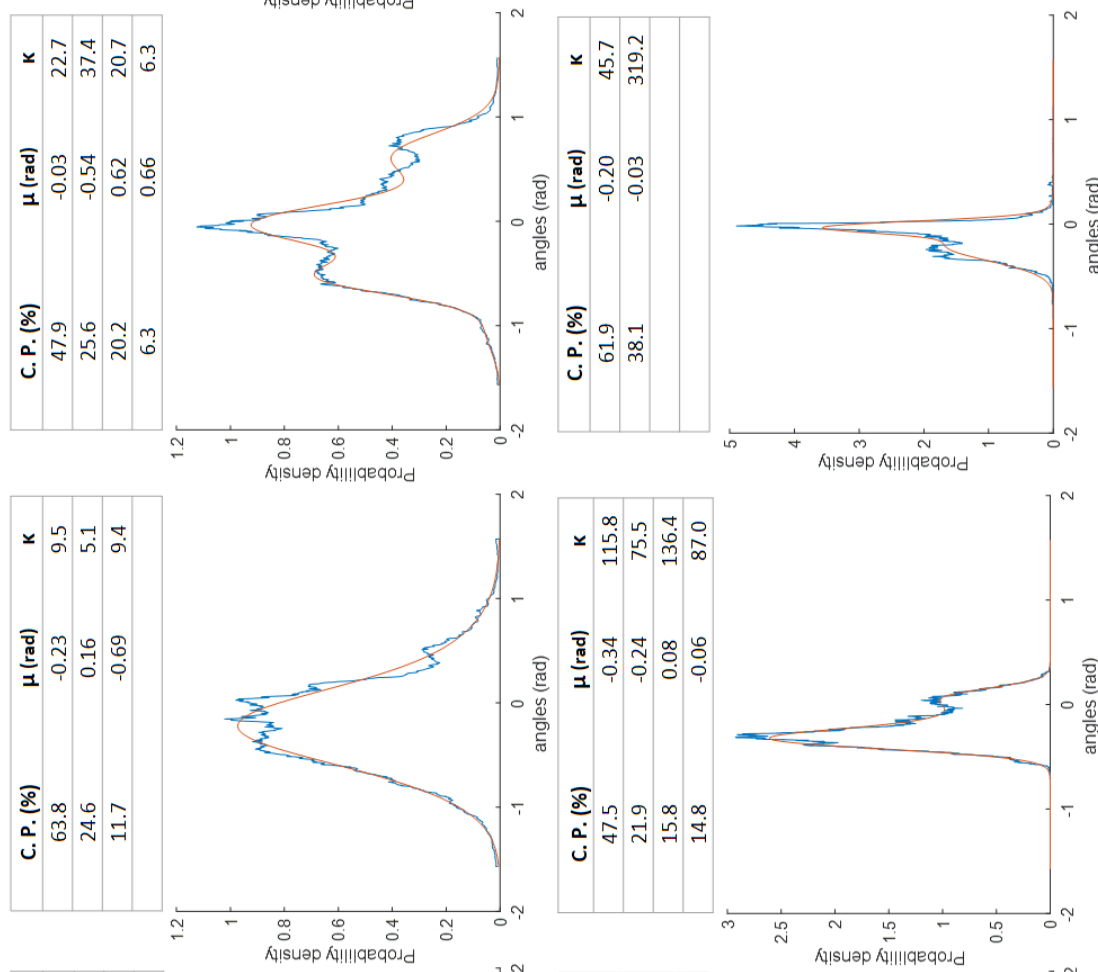

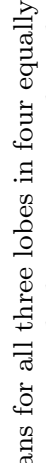
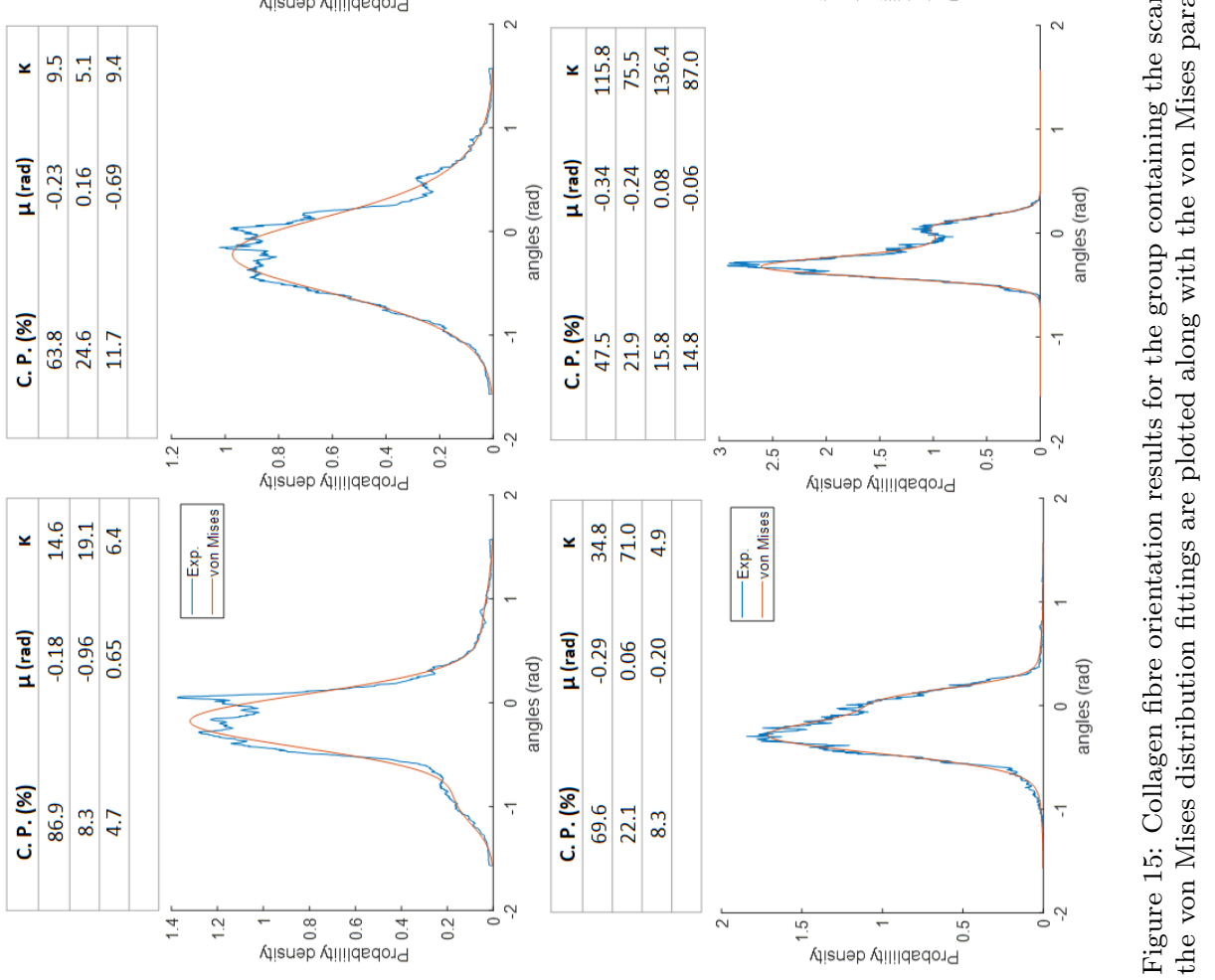

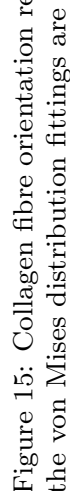


Bio-variability is a well-known factor affecting BV [3] 15]. This also became apparent in this study. The BV scans showed two different microstructural architectures within our samples. In 8 out of 12 cases it was uniformly distributed collagen, having bundles with a mean diameter of 2.92 $\mu \mathrm{m}$ and a single layer. In the other 4 cases, the veins were double layered with bundles having a mean diameter of $48.58 \mu \mathrm{m}$ in the outer layer, while on the second layer the bundles had similar sizes as the ones described in the first microstructure. Samples with this less common architecture were found in the frontal and parietal lobes and only in samples with diameters larger than 3.2 $\mathrm{mm}$. On the other hand, there was one sample with a diameter of $3.76 \mathrm{~mm}$ than had a uniformly distributed collagenous architecture instead of a layered one. In the occipital lobe the largest recorded diameter was $2.7 \mathrm{~mm}$ and all samples had the smaller and evenly distributed bundles of no definitive et al. the reported collagen fibrils had an approximate size of $0.7 \mu \mathrm{m}$ and the collagen bundles ranged between 10-20 $\mu \mathrm{m}^{7}$. In this study, it was not feasible to look for collagen fibrils due to the magnification used.

Regarding the collagen fibre orientation when looking into the group with the sum of all samples, fibre angles show a clear preference in angles close to the axial direction. A good fit was obtained by using a mixture of 3 von Mises distributions on the data. The mean angles $(\mu)$ were at -0.35 , $0.21,-0.02 \mathrm{rad}\left(-20^{\circ}, 12^{\circ},-1^{\circ}\right)$, all very close to the axial direction. When the same data were analysed per block, we saw that the fibres get more concentrated the closer we get to the SSS. Even though it is clear that the collagen fibres do not always have one axially oriented fibre family it is becoming clear, for at least the number of analysed samples in this study, that there is always a main component of fibres that it is axially oriented.

In order to have a known reference coordinate system on the fibre angles of the BV, a uniaxial tension set up was used to make the specimens self-align and then a 50\% uniaxial strain was applied. direction is also an indication that the assumed coordinate system was in agreement with the real axis of the vein.

Using a multiphoton microscope allowed us to perform label-free imaging of the BV. Because of its high hyper-polarizability, non-centrosymmetric and organized structure, collagen generates second harmonics wavelengths when hit with highly focussed laser light. Apart from generating second harmonics, the 850nm laser also excites, according to the 2-photon principle, auto-fluorescent structures that fluoresce and can be detected (mainly) in the backward (epi) direction. The second harmonics signals on the other hand are coherent and propagate predominantly in the forward direction. This was a considerable asset in this study since no staining was needed and all the veins were tested fresh inside a water bath. Therefore no processing was done on the tissue that could affect the collagen architecture.

\subsection{Limitations and future work}

The major limitation of this study is that all the studied samples were acquired from a single human cadaver. The results presented in this study can give valuable insights, as collagen fibre orientation on fresh human bridging veins has never been studied before in a quantitative way. Also, there are previous studies on bridging veins also reporting that the collagen fibres are longitudinally oriented even though they are not doing so in a quantitative way. Nonetheless, more studies will be necessary in the future to ensure that the implementation of these results in finite element modelling is based in generalizable results. 
One has to be careful when using second harmonics imaging due to the fact that it is a polarisation dependent technique. Unavoidably, with horizontal polarisation and with the vessel having a horizontal orientation, the imaging of the horizontally oriented fibres are favoured. On the other hand, the acquired signal was always strong enough to portray the fibres in the other directions sufficiently and our findings agree with the finding in literature on the fact that the fibres in BV are oriented close to the axial direction. Furthermore, the fact that we also detect fibre families with mean angles in steeper angles comes to show that the acquired signal was strong enough to show the full range of fibre directions in BV as it can be seen in sample 11 and sample 14, which are shown in the appendix.

To improve the image quality all the veins were cut open to provide a flat surface. Cutting open the veins could introduce false orientation values and this is why the scans were focused away from the edges of the tissue. Also always a part of the SSS was kept connected to the vein to use it as a clamping area when the tissue was mounted on the tension set up. This means it is unlikely that the fibre orientation values could be affected by the pre scan manipulation. To avoid any stretching on the initial scan, that could change the fibre orientations, the width change of the tissue was measured before and after the initial aligning process and was kept to a minimum.

Furthermore, the scanning area for each vein was several $\mathrm{mm}^{2}$. This meant a large statistical sample of fibre orientations. Due to this large scanning area, differences throughout the length of the BV were seen, showing mostly one fibre family axially oriented closer to the SSS and as progressing closer towards the brain then more fibre families emerged with different mean angles. To scan such a big area, several 3D image stacks were stitched using a Zeiss stitching module. In some cases the quality of the stitching was suboptimum. There are a number of reasons for these suboptimum results. The overlap setting between the stacks in the XY plane could be too low or possible temperature changes caused the lens to "drop" i.e. to slightly change its shape. These stitching inconsistences did not have any effect on the orientation measurements, even though the individual tiles can be visually distinguished the fibre angles in these tiles were not altered.

For practical reasons, the maximum intensity z projections were used instead of the full $3 \mathrm{D} z$ stacks. The analysis of the large datasets of the $\mathrm{z}$ stacks and especially the fitting of the mixture of the von Mises distributions was impossible unless the images were z projections. The maximum intensity z projections could mean that some of the smallest and less pronounced fibres could have been covered by the rest and not taken into account in the orientation analysis but the effect of those fibres should be negligible. Furthermore, in this study the local 2D orientations of the fibres were extracted and therefore no information on out-of-plane fibre orientation of collagen was taken into account. Re-analysing the datasets using hardware with higher computing power could produce results with higher resolution which would be interesting to compare to the current results and in that case, the out-of-plane fibre orientation could also be taken into account.

To the authors knowledge, this is the first study investigating the collagen orientation of fresh human bridging veins, using scanning areas of several $\mathrm{mm}^{2}$ and applying tension while scanning the specimens, either to straighten or to deform, giving insight on the microstructural architecture of the bridging veins. These data can be used to model anisotropy of the tissue.However, to ensure that the results are generalizable, more studies will be necessary in the future.

Further investigation on the fibre recruitment by applying different deformations could give valuable information on the damage properties of bridging veins. Therefore even though it is clear that the collagen fibres do not always have one fibre family as literature seemed to suggest it is becoming clear, for at least the number of analysed samples in this study, that there is also a main component and that it is axially oriented. Furthermore, when the $50 \%$ strain was applied on the 
second scan there was a clear recruitment of fibres towards the direction of the applied strain, i.e. the axial direction. A new study investigating the level of recruitment on different levels of strain could give useful insight on the damage mechanisms in BV under uniaxial strain.

Although one might say that the underlying bio-variability in BV complicates any attempts to draw concrete conclusions and to model this tissue, computational modelling can take these uncertainties into consideration. Having information with high biofidelity along with the full range of possible values is imperative in order for finite element modelling to bring new insights to the understanding of the mechanism behind head impact traumas 21222324.

In this case clear differences in the number of layers and in collagen bundle size, which are related to the diameter size of the $\mathrm{BV}$, could be modelled, following a multiscale approach, to investigate the different loading regimes a BV could undergo when the same loading conditions are applied ${ }^{7}$. In addition fibre angle distributions can be reconstructed by using the reported von Mises parameters and can applied directly on material models [25] [16] and investigate the mechanical response of the veins under injurious loading conditions.

Therefore, in order to shed more light into the aetiology of ASDH due to BV ruptures a higher degree of biofidelity has to be introduced to the FE head models. Introducing anisotropy in the BV material models is crucial along with the geometrical and material biofidelity of the surrounding structures such as the dura, the pia and the cerebrospinal fluid 262 .

\section{Conclusion}

In the present study two main conclusions can be drown. From the examined samples it is most common to have a BV microstructure which is single layered and homogenous with a mean bundle size of $2.92 \mu \mathrm{m}$. In cases the diameter exceeds $3.2 \mathrm{~mm}$ it is possible to have a double layered geometry with a mean bundle size of $48.58 \mu \mathrm{m}$ in the outer layer. Collagen fibre orientation analysis showed that usually there is one axially oriented fibre family, but there can be more than one fibre families in $\mathrm{BV}$ in angles ranging from 1.05 to $-1.05 \mathrm{rad}\left(60^{\circ}\right.$ to $\left.-60^{\circ}\right)$.

Both findings could be directly applied in modelling BV for applications such as accident damage prevention, presurgical planning, etc.

\section{Conflict of interest statement}

The authors have no conflicts of interest arising from the direct applications of this research.

\section{Acknowledgements}

This work was supported by the European Union's Horizon 2020 research and innovation programme under the Marie Skłodowska-Curie grant agreement No. 642662 for HEADS ITN and by a personal FWO fellowship (PDO/ 12), a FWO grant G0C6713N, a FWO grant G.0929.15 and Hercules AKUL/11/ 37 


\section{References}

\section{References}

[1] B. Depreitere, C. Van Lierde, S. Maene, C. Plets, J. V. Sloten, R. Van Audekercke, G. Van der Perre, J. Goffin, Bicycle-related head injury: A study of 86 cases, Accident Analysis and Prevention 36 (4) (2004) 561-567.

[2] Z. Zhou, X. Li, S. Kleiven, Biomechanics of acute subdural hematoma in the elderly : A fluidstructure interaction study (2018) 1-30.

[3] N. Famaey, Z. Ying Cui, G. Umuhire Musigazi, J. Ivens, B. Depreitere, E. Verbeken, J. Vander Sloten, Structural and mechanical characterisation of bridging veins: A review., Journal of the mechanical behavior of biomedical materials 41 (2015) 222-240.

[4] T. Yamashima, R. L. Friede, Why do bridging veins rupture into the virtual subdural space?, Journal of neurology, neurosurgery, and psychiatry 47 (2) (1984) 121-127.

[5] Q. Pang, H. Gregersen, V. Oettingen, J. Astrup, Biomechanical Properties of Porcine Cerebral Bridging Veins with Reference to the Zero-Stress State (2001) 83-90.

[6] J.-R. Vignes, A. Dagain, J. Guerin, D. Liguoro, A hypothesis of cerebral venous system regulation based on a study of the junction between the cortical bridging veins and the superior sagittal sinus. Laboratory investigation., Journal of neurosurgery 107 (6) (2007) 1205-1210.

[7] M. Nierenberger, Y. Re, S. Ahzi, A new multiscale model for the mechanical behavior of vein walls, Journal of the Mechanical Behavior of Biomedical Materials 23 (2013) 32-43.

[8] K. Oka, A. L. Rhoton, M. Barry, R. Rodriguez, Microsurgical Anatomy of the Superficial Veins of the Cerebrum, Forensic Science International 17 (5).

[9] K. L. Monson, W. Goldsmith, N. M. Barbaro, G. T. Manley, Significance of source and size in the mechanical response of human cerebral blood vessels., Journal of biomechanics 38 (4) (2005) 737-744.

[10] D. Garcia, Robust smoothing of gridded data in one and higher dimensions with missing values, Comput. Stat. Data Anal. 54 (4) (2010) 1167-1178.

[11] E. Davies, Machine Vision: Theory, Algorithms and Practicalities, Academic Press, 1990.

[12] J. N. Archana, P. Aishwarya, A Review on the Image Sharpening Algorithms Using Unsharp Masking, Int. J. Eng. Sci. Comp.

[13] Z. Puspoki, M. Storath, D. Sage, M. Unser, Transforms and Operators for Directional Bioimage Analysis: A Survey., Advances in anatomy, embryology, and cell biology 219 (2016) 69-93.

[14] R. Rezakhaniha, A. Agianniotis, J. T. C. Schrauwen, A. Griffa, D. Sage, C. V. C. Bouten, F. N. van de Vosse, M. Unser, N. Stergiopulos, Experimental investigation of collagen waviness and orientation in the arterial adventitia using confocal laser scanning microscopy., Biomechanics and modeling in mechanobiology 11 (3-4) (2012) 461-473. 
[15] C. Brockmann, S. C. Kunze, P. Schmiedek, C. Groden, J. Scharf, Variations of the superior sagittal sinus and bridging veins in human dissections and computed tomography venography, Journal of Clinical Imaging 36 (2) (2012) 85-89. URL http://dx.doi.org/10.1016/j.clinimag.2011.05.003

[16] A. Ni Annaidh, K. Bruy, M. Destrade, M. D. Gilchrist, C. Maurini, M. Ottenio, G. Saccomandi, Automated Estimation of Collagen Fibre Dispersion in the Dermis and its Contribution to the Anisotropic Behaviour of Skin, Annals of Biomedical Engineering (2012) 1-37.

[17] J. Bentley, Modelling Circular Data Using a Mixture of von Mises and Uniform Distributions.

[18] I. S. Dhillon, S. Sra, Modeling Data using Directional Distributions (2003) 1-21.

[19] J. Vesely, L. Horny, H. Chlup, R. Zitny, Collagen orientation and waviness within the vein wall, Computational Plasticity XI - Fundamentals and Applications, COMPLAS XI.

[20] M. Nierenberger, G. Fargier, S. Ahzi, Y. Rémond, Evolution of the three-dimensional collagen structure in vascular walls during deformation : an in situ mechanical testing under multiphoton microscopy observation, Biomechanics and Modeling in Mechanobiology (2015) 693-702. URL http://dx.doi.org/10.1007/s10237-014-0630-4

[21] Y. Cai, S. Wu, W. Zhao, Z. Li, Z. Wu, S. Ji, Concussion classification via deep learning using whole-brain white matter fiber strains, PLoS One (2018) 1-21.

[22] W. Becker, J. Rowson, J. E. Oakley, A. Yoxall, G. Manson, K. Worden, Bayesian sensitivity analysis of a model of the aortic valve, Journal of Biomechanics 44 (8) (2011) 1499-1506. URL http://dx.doi.org/10.1016/j.jbiomech.2011.03.008

[23] W. Becker, J. E. Oakley, C. Surace, P. Gili, J. Rowson, K. Worden, Bayesian sensitivity analysis of a nonlinear finite element model, Mechanical Systems and Signal Processing 32 (2012) 1831. URL http://dx.doi.org/10.1016/j.ymssp.2012.03.009

[24] W. Becker, K. Worden, J. Rowson, Bayesian sensitivity analysis of bifurcating nonlinear models, Mechanical Systems and Signal Processing 34 (1-2) (2013) 57-75. URL http://dx.doi .org/10.1016/j.ymssp.2012.05.010

[25] Y. Wang, S. Son, S. M. Swartz, N. C. Goulbourne, International Journal of Solids and Structures A mixed Von Mises distribution for modeling soft biological tissues with two distributed fiber properties, International Journal of Solids and Structures 49 (21) (2012) 2914-2923. URL http://dx.doi.org/10.1016/j.ijsolstr.2012.04.004

[26] D. D. Kegel, J. Vastmans, H. Fehervary, B. Depreitere, J. V. Sloten, Journal of the Mechanical Behavior of Biomedical Materials Biomechanical characterization of human dura mater, Journal of the Mechanical Behavior of Biomedical Materials 79 (December 2017) (2018) 122-134. URL https://doi.org/10.1016/j.jmbbm.2017.12.023 


\section{Appendix}

\section{- Detailed results for all individual scans and groups}

The remaining results from the microscopic analysis of the BV are placed in this section of the appendix. In order to maximise the amount of detail that can be seen in the scans and figures, the microscopy colour maps and the analysis in blocks are displayed in landscape.

Therefore instead of displaying by sample the results are displayed by category, first the color maps then the entire length analysis and then the analysis in blocks.

9.1. Fibre orientation analysis results summary 
Table 3: Summary table of the fibre orientation analysis - Entire scan length, $0 \%$ strain and $50 \%$ strain

\begin{tabular}{|c|c|c|c|c|c|c|}
\hline Sample & \multicolumn{3}{|c|}{$0 \%$ strain } & \multicolumn{3}{|c|}{$50 \%$ strain } \\
\hline \multirow[t]{3}{*}{2} & 60.0 & -0.59 & 40.0 & 64.4 & -0.09 & 54.0 \\
\hline & 40.0 & -0.30 & 9.2 & 23.4 & 0.13 & 412.9 \\
\hline & & & & 9.8 & 0.13 & 56.4 \\
\hline \multirow[t]{2}{*}{3} & 66.5 & -0.04 & 170.9 & 85.1 & 0.12 & 251.4 \\
\hline & 33.5 & -0.24 & 8.0 & 14.9 & 0.14 & 14.0 \\
\hline \multirow[t]{4}{*}{4} & 51.7 & 0.16 & 4.7 & 43.3 & -0.17 & 12.4 \\
\hline & 48.3 & 0.34 & 11.0 & 30.5 & 0.50 & 5.9 \\
\hline & & & & 24.9 & 0.48 & 40.8 \\
\hline & & & & 1.4 & -0.95 & 16.5 \\
\hline \multirow[t]{3}{*}{6} & 81.4 & 0.00 & 68.0 & 57.1 & 0.03 & 106.7 \\
\hline & 9.4 & -0.20 & 9.8 & 24.9 & 0.22 & 80.7 \\
\hline & 9.2 & 0.49 & 87.8 & 17.9 & 0.06 & 8.7 \\
\hline \multirow[t]{2}{*}{9} & 72.7 & 0.02 & 12.3 & 85.8 & 0.00 & 63.4 \\
\hline & 27.3 & 0.05 & 116.9 & 14.2 & -0.05 & 10.8 \\
\hline \multirow[t]{3}{*}{10} & 92.7 & -0.19 & 14.2 & 36.5 & 0.17 & 94.4 \\
\hline & 7.3 & -0.87 & 16.9 & 35.7 & -0.12 & 52.5 \\
\hline & & & & 27.8 & 0.45 & 61.2 \\
\hline \multirow[t]{4}{*}{11} & 45.9 & 0.65 & 11.8 & 60.3 & 0.04 & 26.8 \\
\hline & 38.4 & -0.29 & 6.8 & 22.8 & 0.17 & 4.4 \\
\hline & 15.1 & -0.16 & 360.5 & 16.9 & 0.56 & 37.6 \\
\hline & 0.6 & -1.45 & 236.0 & & & \\
\hline \multirow[t]{3}{*}{12} & 76.3 & -0.48 & 30.0 & 62.4 & -0.06 & 33.5 \\
\hline & 23.7 & -0.19 & 4.4 & 24.3 & 0.05 & 221.8 \\
\hline & & & & 13.3 & 0.24 & 7.5 \\
\hline \multirow[t]{3}{*}{13} & 41.9 & 0.30 & 8.2 & 56.8 & 0.15 & 19.9 \\
\hline & 29.7 & 0.13 & 60.2 & 41.7 & 0.12 & 85.3 \\
\hline & 28.4 & -0.16 & 67.1 & 1.5 & -0.03 & 2.3 \\
\hline \multirow[t]{3}{*}{14} & 50.7 & -0.92 & 19.9 & 60.4 & -0.07 & 18.8 \\
\hline & 32.5 & 0.22 & 85.3 & 38.3 & -0.51 & 12.8 \\
\hline & 16.8 & -0.23 & 2.3 & 1.3 & 0.93 & 5.5 \\
\hline \multirow[t]{2}{*}{15} & 59.2 & -0.26 & 35.2 & 97.3 & -0.37 & 27.2 \\
\hline & 40.8 & 0.06 & 4.8 & 2.7 & -0.36 & 2.8 \\
\hline \multirow[t]{3}{*}{16} & 50.6 & 0.29 & 32.2 & 70.9 & 0.11 & 50.2 \\
\hline & 38.7 & -0.22 & 29.2 & 29.1 & 0.21 & 7.2 \\
\hline & 10.7 & 0.09 & 3.9 & & & \\
\hline
\end{tabular}


Table 4: Summary table of the fibre orientation analysis - 4 blocks of equal scan length, $0 \%$ strain

\begin{tabular}{|c|c|c|c|c|c|c|c|c|c|c|c|c|}
\hline \multirow[b]{2}{*}{ Sample } & \multicolumn{3}{|c|}{ Block 1} & \multicolumn{3}{|c|}{ Block 2} & \multicolumn{3}{|c|}{ Block 3} & \multicolumn{3}{|c|}{ Block 4} \\
\hline & C.P. $(\%)$ & $\mu(\mathrm{rad})$ & $\kappa$ & C.P. $(\%)$ & $\mu(\mathrm{rad})$ & $\kappa$ & C.P. $(\%)$ & $\mu(\mathrm{rad})$ & $\kappa$ & C.P. $(\%)$ & $\mu(\mathrm{rad})$ & $\kappa$ \\
\hline \multirow[t]{2}{*}{2} & 57.0 & -0.64 & 21.8 & 89.5 & -0.63 & 37.7 & 70.0 & -0.63 & 53.5 & 98.1 & -0.33 & 23.4 \\
\hline & 42.6 & 0.04 & 12.6 & 10.5 & -0.10 & 4.9 & 30.0 & -0.33 & 31.4 & 1.9 & 0.03 & 6.8 \\
\hline \multirow[t]{4}{*}{3} & 75.8 & -0.21 & 11.9 & 63.3 & -0.05 & 142.0 & 74.0 & -0.03 & 136.3 & 90.2 & -0.04 & 197.0 \\
\hline & 22.3 & -0.02 & 456.8 & 36.7 & -0.23 & 4.9 & 15.5 & -0.18 & 101.0 & 9.8 & -0.24 & 5.5 \\
\hline & 1.9 & 0.82 & 12.7 & & & & 6.2 & -0.04 & 4.7 & & & \\
\hline & & & & & & & 4.3 & -0.66 & 23.2 & & & \\
\hline \multirow[t]{3}{*}{4} & 100.0 & 0.10 & 44.2 & 63.7 & -0.19 & 32.4 & 49.9 & 0.41 & 32.9 & 100.0 & 0.34 & 8.1 \\
\hline & & & & 36.3 & -0.15 & 7.7 & 45.8 & 0.09 & 5.4 & & & \\
\hline & & & & & & & 4.4 & 0.93 & 100.0 & & & \\
\hline \multirow[t]{4}{*}{6} & 71.3 & 0.07 & 340.3 & 50.8 & 0.00 & 75.2 & 55.3 & -0.11 & 10.4 & 95.6 & -0.08 & 91.2 \\
\hline & 28.7 & -0.01 & 27.4 & 23.4 & 0.49 & 100.4 & 23.5 & -0.03 & 276.2 & 4.4 & -0.18 & 3.3 \\
\hline & & & & 22.5 & 0.21 & 177.6 & 21.2 & 0.44 & 100.9 & & & \\
\hline & & & & 3.2 & -0.62 & 14.6 & & & & & & \\
\hline \multirow[t]{3}{*}{9} & 43.9 & 0.38 & 48.6 & 61.7 & -0.01 & 39.3 & 65.5 & 0.07 & 93.4 & 77.6 & -0.01 & 81.2 \\
\hline & 37.0 & 0.11 & 78.2 & 25.7 & -0.42 & 54.4 & 34.5 & 0.1 & 16.0 & 22.4 & -0.03 & 17.3 \\
\hline & 19.1 & -0.23 & 31.9 & 12.5 & 0.29 & 29.1 & & & & & & \\
\hline \multirow[t]{4}{*}{10} & 51.5 & -0.36 & 32.2 & 71.6 & -0.06 & 64.0 & 50.9 & -0.62 & 10.6 & 52.1 & -0.66 & 11.5 \\
\hline & 32.5 & 0.12 & 4.4 & 27.4 & -0.51 & 5.4 & 30.9 & 0.03 & 66.2 & 28.3 & 0.49 & 6.9 \\
\hline & 16.0 & -0.80 & 5.4 & 1.0 & 1.13 & 30.2 & 15.4 & -0.45 & 53.0 & 16.5 & -0.24 & 19.4 \\
\hline & & & & & & & 2.8 & 0.19 & 4.3 & 3.1 & -1.58 & 99.2 \\
\hline \multirow[t]{4}{*}{11} & 41.6 & -0.50 & 4.4 & 61.9 & -0.08 & 3.8 & 68.4 & 0.77 & 47.6 & 52.1 & 0.30 & 5.6 \\
\hline & 35.8 & 0.76 & 10.1 & 38.1 & 0.75 & 16.3 & 28.3 & 0.16 & 17.4 & 47.9 & 0.76 & 55.7 \\
\hline & 20.3 & -0.18 & 288.6 & & & & 3.3 & 0.42 & 3.6 & & & \\
\hline & 2.3 & 1.45 & 152.1 & & & & & & & & & \\
\hline \multirow[t]{3}{*}{12} & 89.3 & -0.36 & 61.5 & 88.7 & -0.41 & 27.0 & 67.8 & -0.36 & 29.6 & 80.6 & -0.51 & 62.2 \\
\hline & 10.7 & 0.24 & 4.0 & 7.7 & -0.02 & 133.2 & 30.9 & -0.60 & 194.7 & 17.9 & -0.23 & 7.9 \\
\hline & & & & 3.6 & -0.05 & 5.1 & 1.2 & 0.04 & 14.4 & 1.5 & -0.98 & 124.7 \\
\hline \multirow[t]{3}{*}{13} & 78.9 & -0.12 & 30.7 & 57.1 & -0.13 & 48.0 & 61.2 & 0.33 & 7.7 & 97.3 & 0.10 & 29.3 \\
\hline & 21.1 & 0.14 & 10.6 & 23.4 & 0.46 & 48.2 & 38.8 & 0.73 & 97.8 & 2.7 & 0.64 & 1.9 \\
\hline & & & & 19.6 & 0.17 & 84.1 & & & & & & \\
\hline \multirow[t]{4}{*}{14} & 89.3 & -0.95 & 19.5 & 86.2 & -0.98 & 21.3 & 77.1 & -0.57 & 9.0 & 64.5 & -0.1 & 7.8 \\
\hline & 8.2 & -0.32 & 10.7 & 12.1 & -0.31 & 20.4 & 16.0 & -1.17 & 34.2 & 27.9 & 0.60 & 12.8 \\
\hline & 2.5 & 1.35 & 32.5 & 1.6 & 1.36 & 24.3 & 7.0 & 1.15 & 13.7 & 5.4 & -1.32 & 37.9 \\
\hline & & & & & & & & & & 2.2 & 1.36 & 58.8 \\
\hline \multirow[t]{4}{*}{15} & 74.3 & -0.24 & 60.3 & 76.3 & -0.28 & 25.1 & 54.3 & -0.01 & 5.3 & 47.5 & 0.25 & 11.8 \\
\hline & 25.7 & 0.01 & 12.8 & 23.7 & 0.14 & 3.2 & 45.7 & -0.27 & 31.7 & 33.9 & -0.22 & 18.2 \\
\hline & & & & & & & & & & 13.4 & 0.55 & 59.3 \\
\hline & & & & & & & & & & 5.1 & 0.16 & 4.2 \\
\hline \multirow[t]{3}{*}{16} & 66.4 & -0.30 & 64.4 & 54.7 & 0.10 & 19.6 & 57.6 & 0.13 & 12.1 & 50.6 & 0.26 & 27.7 \\
\hline & 33.6 & -0.13 & 11.2 & 45.3 & 0.30 & 73.5 & 34.8 & 0.35 & 92.9 & 41.5 & -0.21 & 24.9 \\
\hline & 29.1 & 0.21 & 7.2 & & & & 7.6 & -0.19 & 5.2 & 7.9 & 0.13 & 3.2 \\
\hline
\end{tabular}


Table 5: Summary table of the fibre orientation analysis - 4 blocks of equal scan length, $50 \%$ strain

\begin{tabular}{|c|c|c|c|c|c|c|c|c|c|c|c|c|}
\hline \multirow[b]{2}{*}{ Sample } & \multicolumn{3}{|c|}{ Block 1} & \multicolumn{3}{|c|}{ Block 2} & \multicolumn{3}{|c|}{ Block 3} & \multicolumn{3}{|c|}{ Block 4} \\
\hline & C.P. $(\%)$ & $\mu(\mathrm{rad})$ & $\kappa$ & C.P. $(\%)$ & $\mu(\mathrm{rad})$ & $\kappa$ & C.P. $(\%)$ & $\mu(\mathrm{rad})$ & $\kappa$ & C.P. $(\%)$ & $\mu(\mathrm{rad})$ & $\kappa$ \\
\hline \multirow[t]{3}{*}{2} & 97.7 & -0.21 & 18.9 & 47.2 & -0.34 & 119.6 & 61.9 & -0.20 & 45.7 & 64.1 & -0.04 & 350.4 \\
\hline & 2.3 & 0.03 & 3.2 & 33.0 & -0.19 & 60.0 & 38.1 & -0.03 & 319.2 & 35.9 & -0.17 & 27.4 \\
\hline & & & & 19.8 & 0.07 & 122.2 & & & & & & \\
\hline \multirow[t]{2}{*}{3} & 76.4 & -0.09 & 256.9 & 78.1 & -0.09 & 348.0 & 99.3 & -0.07 & -281.2 & 97.1 & -0.07 & 195.7 \\
\hline & 23.6 & -0.06 & 36.1 & 21.9 & -0.04 & 10.8 & 0.7 & 0.44 & 91.0 & 2.9 & 0.10 & 10.3 \\
\hline \multirow[t]{3}{*}{4} & 94.9 & -0.15 & 21.0 & 69.4 & -0.05 & 9.9 & 81.8 & 0.23 & 19.2 & 92.3 & -0.36 & 16.0 \\
\hline & 5.1 & 0.31 & 150.1 & 23.4 & 0.38 & 89.0 & 14.1 & 0.41 & 376.2 & 7.7 & 0.77 & 15.0 \\
\hline & & & & 7.3 & 0.68 & 16.7 & 4.2 & 0.80 & 201.0 & & & \\
\hline \multirow[t]{3}{*}{6} & 80.8 & 0.08 & 91.4 & 92.3 & 0.10 & 96.6 & 48.5 & 0.03 & 206.5 & 47.0 & -0.02 & 145.8 \\
\hline & 19.2 & 0.04 & 7.0 & 7.7 & 0.11 & 5.5 & 34.4 & 0.23 & 117.4 & 38.5 & 0.08 & 10.2 \\
\hline & & & & & & & 17.1 & 0.07 & 12.5 & 14.5 & 0.23 & 110.8 \\
\hline \multirow[t]{3}{*}{9} & 53.4 & -0.05 & 27.1 & 65.1 & -0.08 & 119.7 & 67.0 & -0.12 & 24.9 & 38.1 & 0.1 & 29.1 \\
\hline & 46.6 & -0.02 & 152.1 & 34.9 & -0.21 & 14.8 & 33.0 & -0.19 & 253.8 & 35.7 & -0.16 & 194.2 \\
\hline & & & & & & & & & & 26.2 & -0.21 & 15.0 \\
\hline \multirow[t]{4}{*}{10} & 79.9 & -0.40 & 72.9 & 46.6 & -0.22 & 24.6 & 36.2 & -0.12 & 129.2 & 98.0 & 0.21 & 53.3 \\
\hline & 11.0 & -0.70 & 619.5 & 44.7 & -0.36 & 88.2 & 31.6 & 0.10 & 98.7 & 2.0 & 0.08 & 2.3 \\
\hline & 8.2 & -0.34 & 11.7 & 7.4 & -0.68 & 40.1 & 18.6 & 0.33 & 126.0 & & & \\
\hline & 1.0 & -0.35 & 3.5 & 1.3 & -0.51 & 3.0 & 13.2 & -0.27 & 45.4 & & & \\
\hline \multirow[t]{3}{*}{11} & 60.3 & -0.17 & 23.0 & 70.5 & 0.16 & 9.9 & 51.2 & 0.43 & 39.6 & 92.9 & 0.16 & 8.9 \\
\hline & 37.9 & 0.51 & 13.0 & 27.7 & -0.48 & 12.7 & 28.1 & -0.04 & 71.2 & 5.3 & -0.23 & 193.2 \\
\hline & 1.7 & -1.06 & 19.1 & 1.7 & -1.35 & 43.0 & 20.8 & 0.19 & 7.8 & 1.8 & -1.12 & 12.7 \\
\hline \multirow[t]{2}{*}{12} & 84.4 & -0.25 & 40.2 & 53.6 & -0.1 & 12.3 & 79.4 & -0.15 & 143.0 & 77.3 & -0.29 & 54.5 \\
\hline & 15.6 & 0.21 & 40.0 & 46.4 & -0.15 & 150.6 & 20.6 & -0.22 & 15.8 & 22.7 & -0.04 & 9.3 \\
\hline \multirow[t]{3}{*}{13} & 56.8 & -0.04 & 84.3 & 56.6 & 0.02 & 25.6 & 88.4 & 0.03 & 42.1 & 94.3 & 0.10 & 154.9 \\
\hline & 43.2 & -0.07 & 10.0 & 27.4 & 0.28 & 127.5 & 9.5 & 0.52 & 166.6 & 5.7 & 0.21 & 5.0 \\
\hline & & & & 16.0 & 0.56 & 53.7 & 2.1 & -0.69 & 17.2 & & & \\
\hline \multirow[t]{3}{*}{14} & 97.7 & -0.52 & 10.4 & 70.1 & -0.58 & 8.8 & 66.7 & -0.16 & 13.6 & 85.8 & -0.12 & 20.9 \\
\hline & 2.3 & 1.07 & 11.2 & 27.2 & -0.29 & 37.1 & 23.7 & -0.75 & 11.9 & 12.5 & -0.69 & 6.8 \\
\hline & & & & 2.8 & 1.01 & 7.9 & 9.6 & -1.05 & 95.8 & 1.8 & 1.03 & 16 \\
\hline \multirow[t]{3}{*}{15} & 70.0 & -0.27 & 56.0 & 80.9 & -0.50 & 49.5 & 94.6 & -0.39 & 45.7 & 89.9 & -0.24 & 15.0 \\
\hline & 30.0 & -0.44 & 13.0 & 19.1 & -0.31 & 8.6 & 5.4 & -0.24 & 8.7 & 9.0 & -0.87 & 20.7 \\
\hline & & & & & & & & & & 1.0 & 1.47 & 193.6 \\
\hline \multirow[t]{2}{*}{16} & 73.8 & -0.02 & 72.0 & 87.0 & 0.02 & 41.1 & 73.9 & -0.04 & 34.7 & 85.6 & -0.01 & 67.7 \\
\hline & 26.2 & 0.12 & 7.9 & 13.0 & -0.02 & 5.6 & 26.1 & 0.14 & 7.8 & 14.4 & 0.05 & 3.5 \\
\hline
\end{tabular}




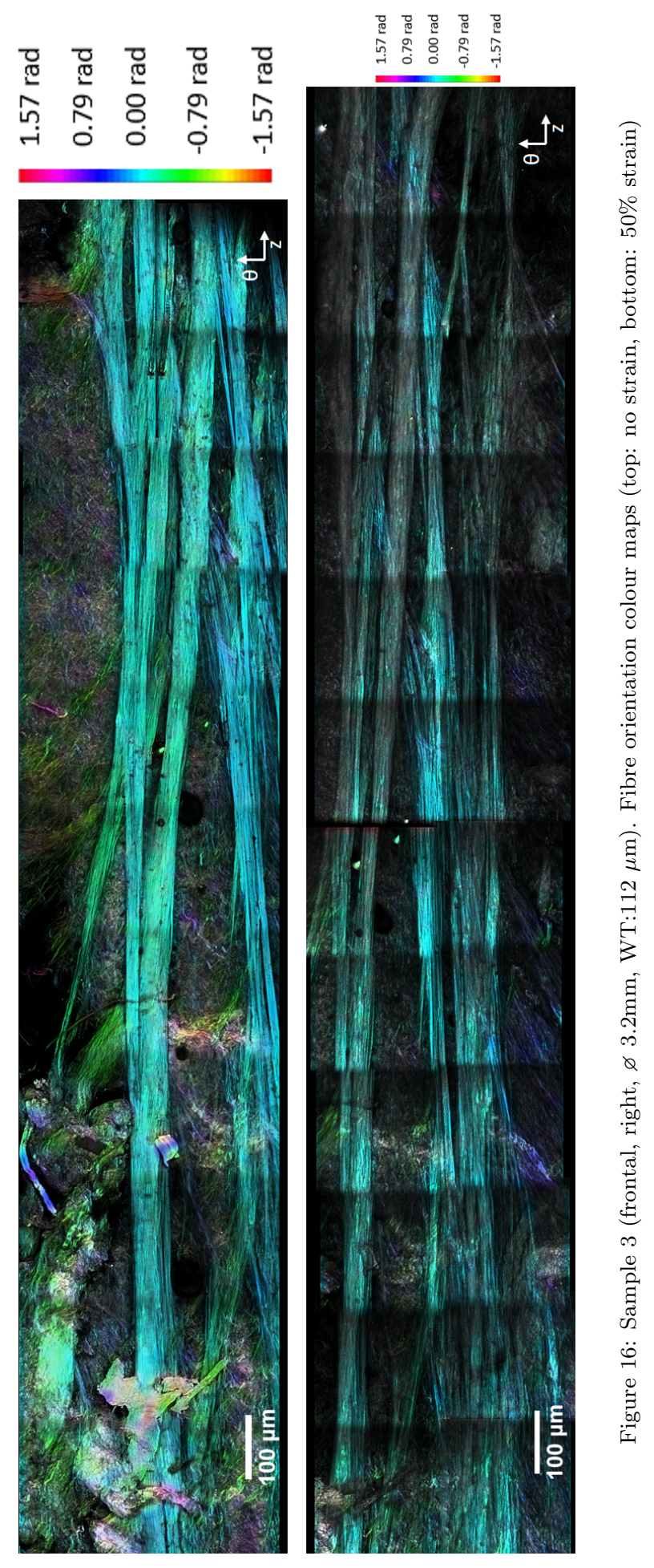




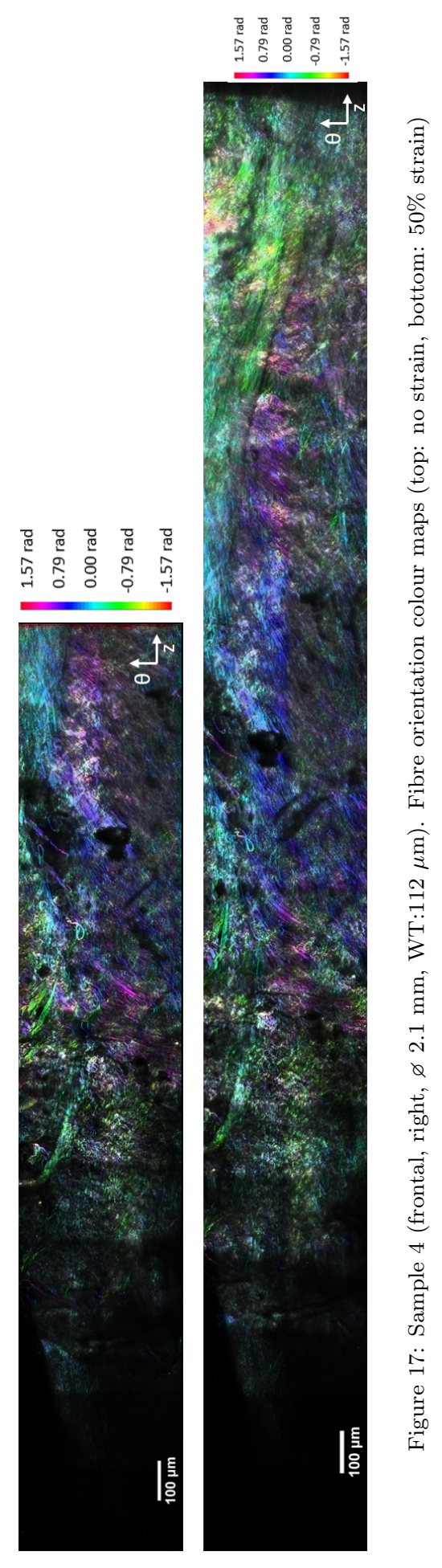



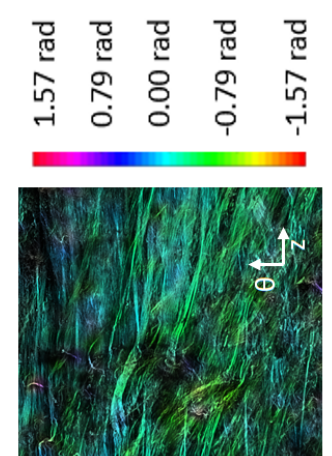

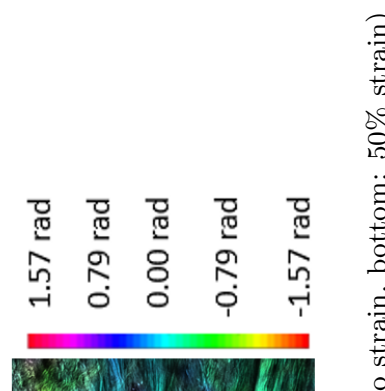

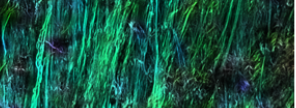

1) (1)

(1)
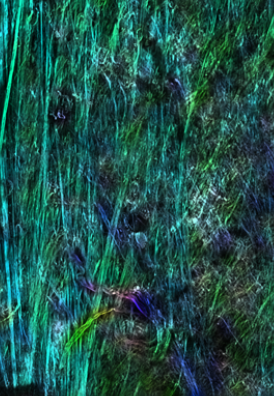

11.

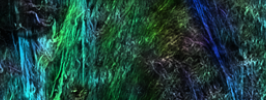

4)

Now

19.

(1) 62

74.06

Y...

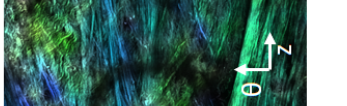

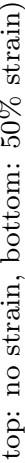
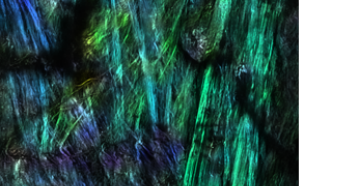

6

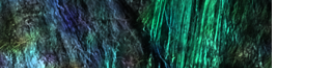

Nind

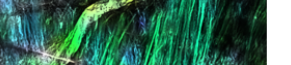

3. (1) oy)
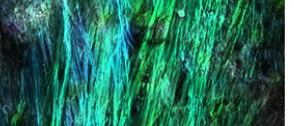

N 11$)$

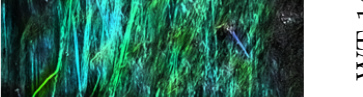

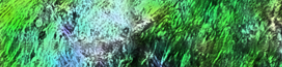

in 110

-

(2x)

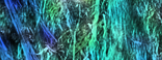

n+ond

(2) $1 \frac{5}{8}$
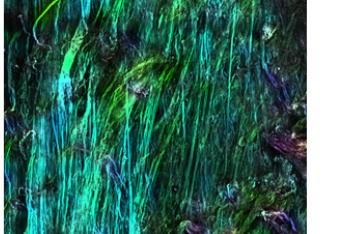

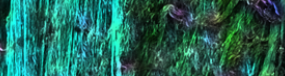
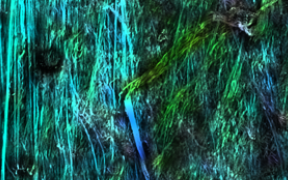

$40-1-x$

4.

1 है 


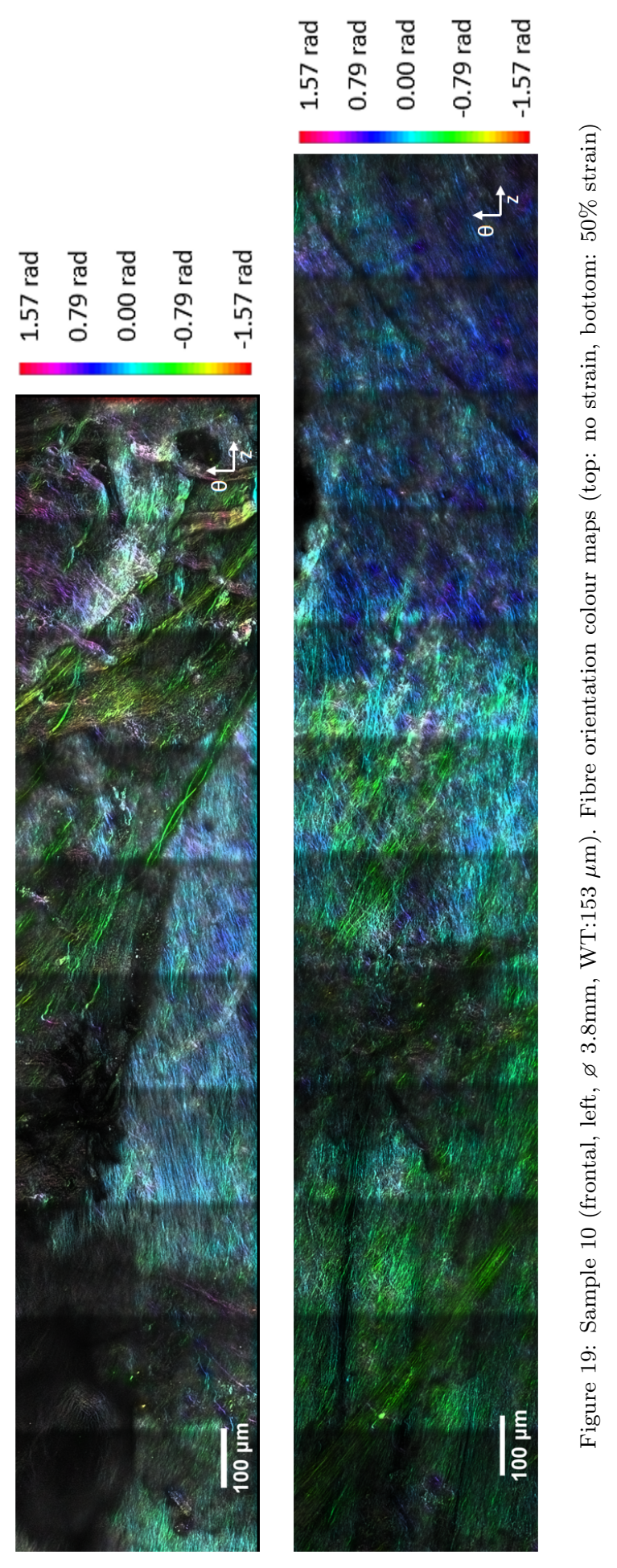




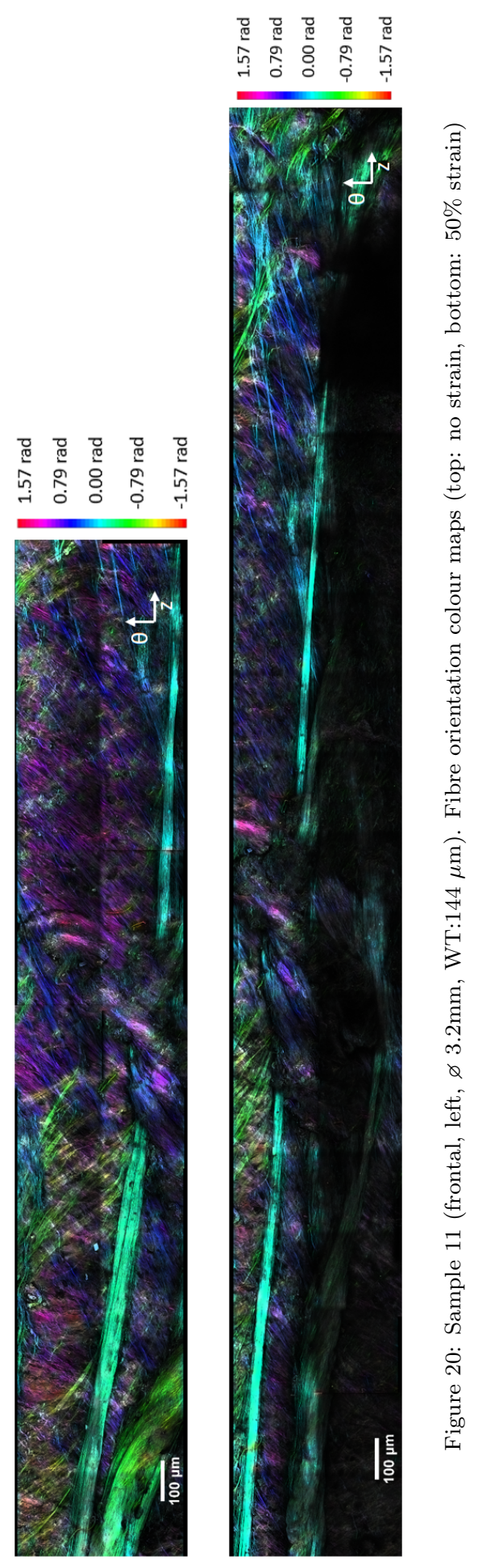




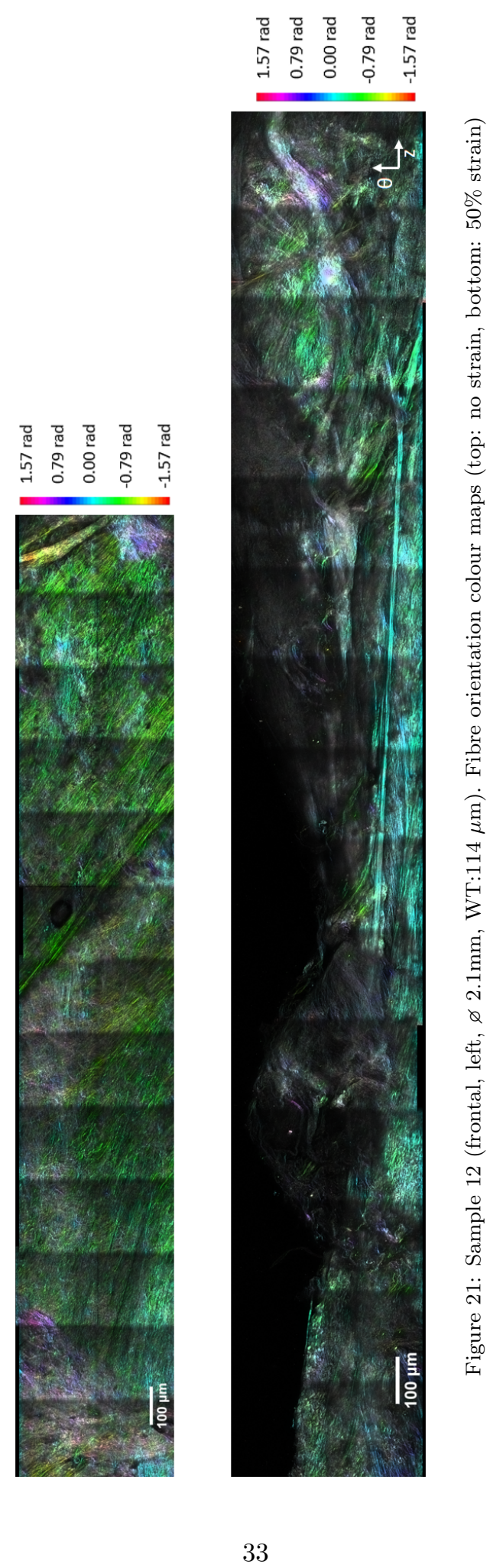




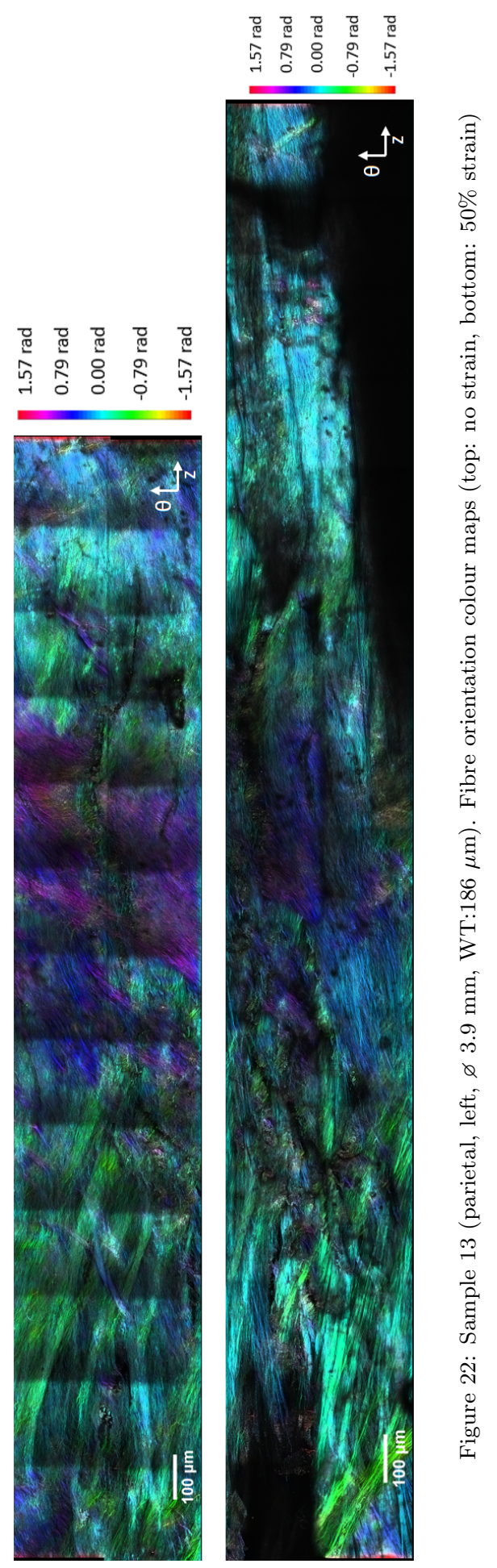




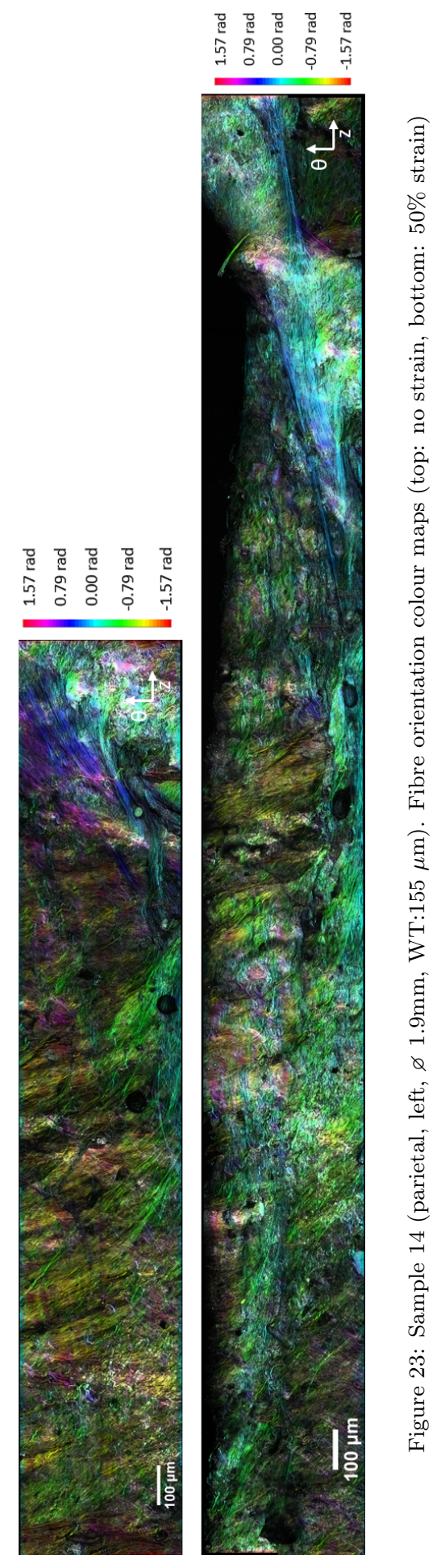




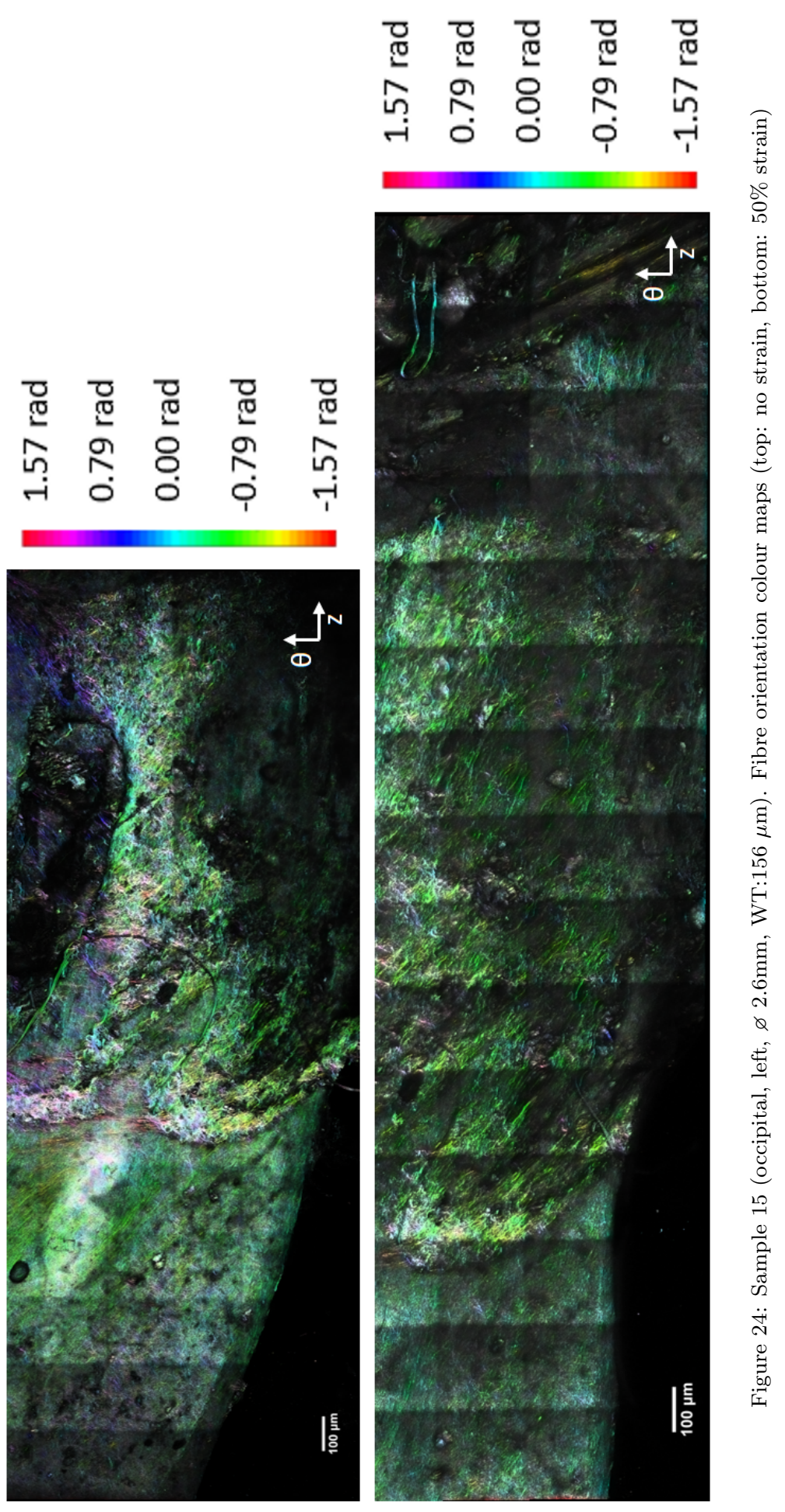




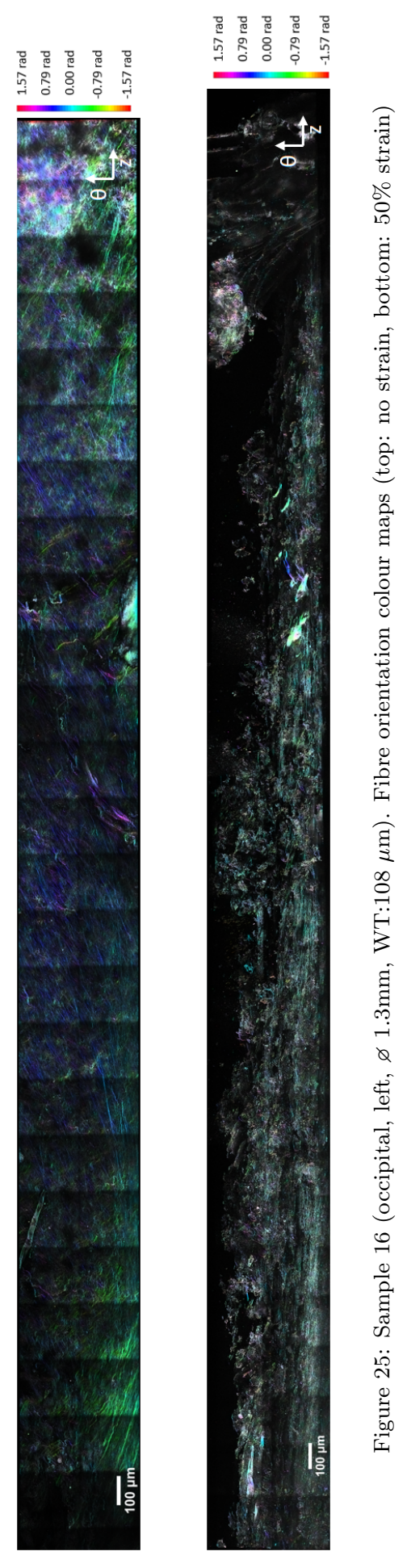




\begin{tabular}{|ccc|}
\hline C. P. (\%) & $\boldsymbol{\mu}$ (rad) & K \\
\hline 66.5 & -0.04 & 170.9 \\
\hline 33.5 & -0.24 & 8.0 \\
\hline & & \\
\hline
\end{tabular}

\begin{tabular}{|ccc|}
\hline C. P. (\%) & $\boldsymbol{\mu}$ (rad) & K \\
\hline 85.1 & 0.12 & 251.4 \\
\hline 14.9 & 0.14 & 14.0 \\
\hline & & \\
\hline
\end{tabular}
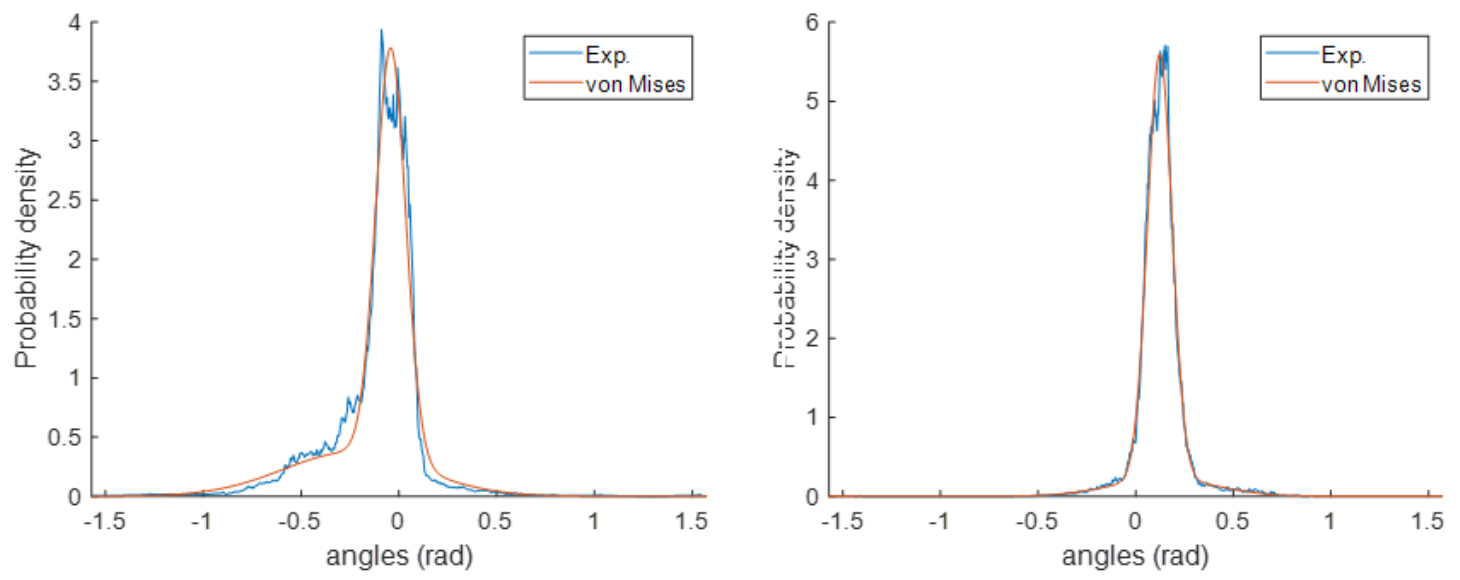

Figure 26: Sample 3, Entire scan analysis - fibre orientations with von Mises fittings (left: no strain, right: $50 \%$ strain) 


\begin{tabular}{|ccc|}
\hline C. P. (\%) & $\boldsymbol{\mu}$ (rad) & K \\
\hline 51.7 & 0.16 & 4.7 \\
\hline 48.3 & 0.34 & 11.0 \\
\hline & & \\
\hline & & \\
\hline
\end{tabular}

\begin{tabular}{|ccc|}
\hline C. P. (\%) & $\boldsymbol{\mu}$ (rad) & K \\
\hline 43.3 & -0.17 & 12.4 \\
\hline 30.5 & 0.50 & 5.9 \\
\hline 24.9 & 0.48 & 40.8 \\
\hline 1.4 & -0.95 & 16.5 \\
\hline
\end{tabular}
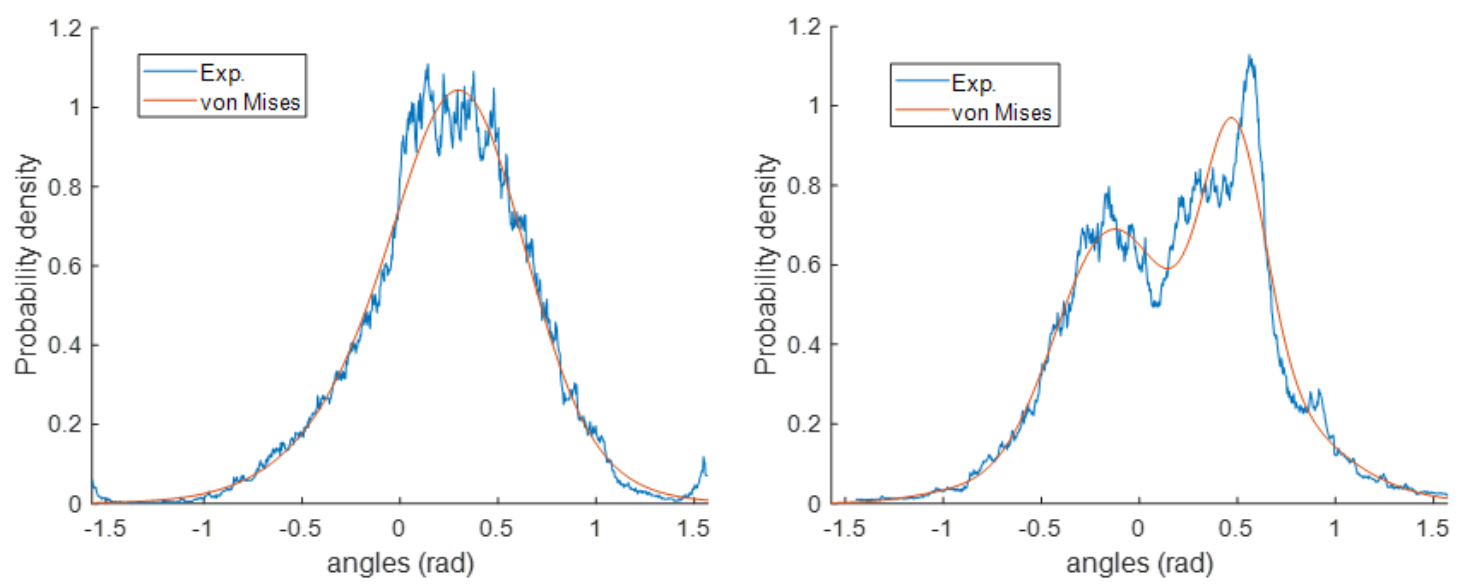

Figure 27: Sample 4, Entire scan analysis - fibre orientations with von Mises fittings (left: no strain, right: $50 \%$ strain) 


\begin{tabular}{|ccc|}
\hline C. P. (\%) & $\boldsymbol{\mu}$ (rad) & K \\
\hline 72.7 & 0.02 & 12.3 \\
\hline 27.3 & 0.05 & 116.9 \\
\hline & & \\
\hline
\end{tabular}

\begin{tabular}{|ccc|}
\hline C. P. (\%) & $\boldsymbol{\mu}$ (rad) & K \\
\hline 85.8 & 0.00 & 63.4 \\
\hline 14.2 & -0.05 & 10.8 \\
\hline
\end{tabular}
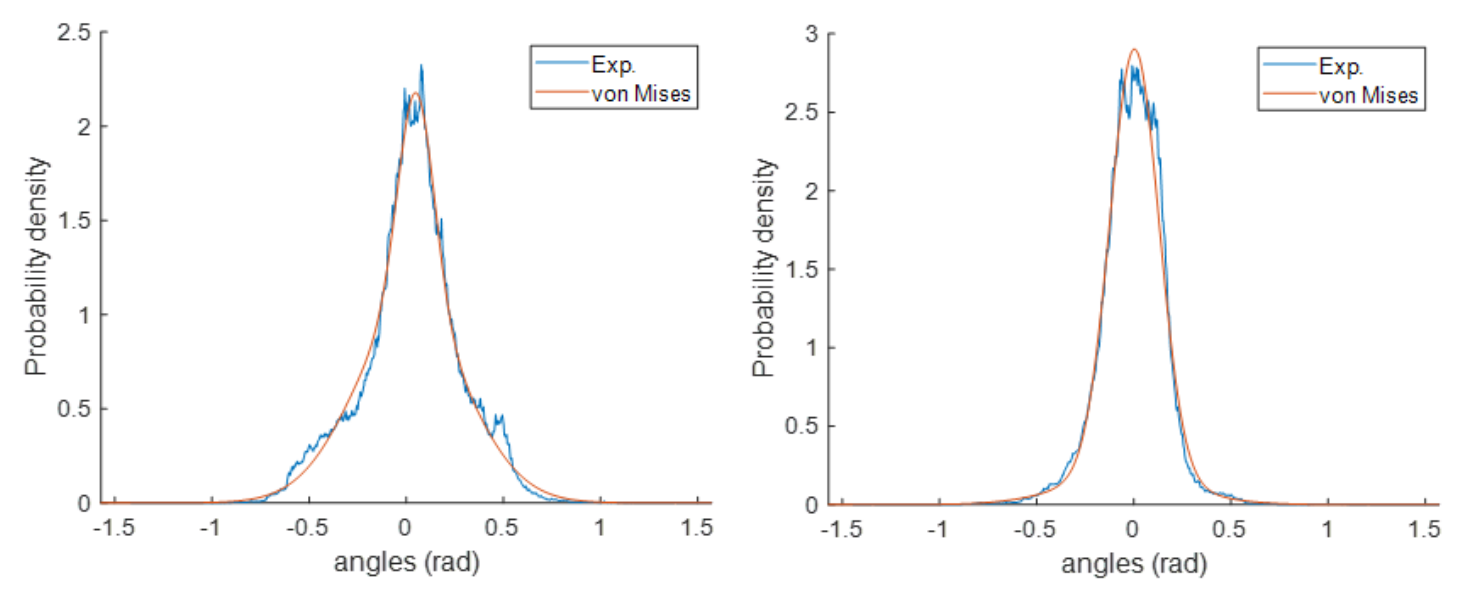

Figure 28: Sample 9, Entire scan analysis - fibre orientations with von Mises fittings (left: no strain, right: $50 \%$ strain)

\begin{tabular}{|ccc|}
\hline C. P. (\%) & $\boldsymbol{\mu}$ (rad) & K \\
\hline 92.7 & -0.19 & 14.2 \\
\hline 7.3 & -0.87 & 16.9 \\
\hline & & \\
\hline
\end{tabular}

\begin{tabular}{|ccc|}
\hline C. P. (\%) & $\boldsymbol{\mu}$ (rad) & K \\
\hline 36.5 & 0.17 & 94.4 \\
\hline 35.7 & -0.12 & 52.5 \\
\hline 27.8 & 0.45 & 61.2 \\
\hline
\end{tabular}
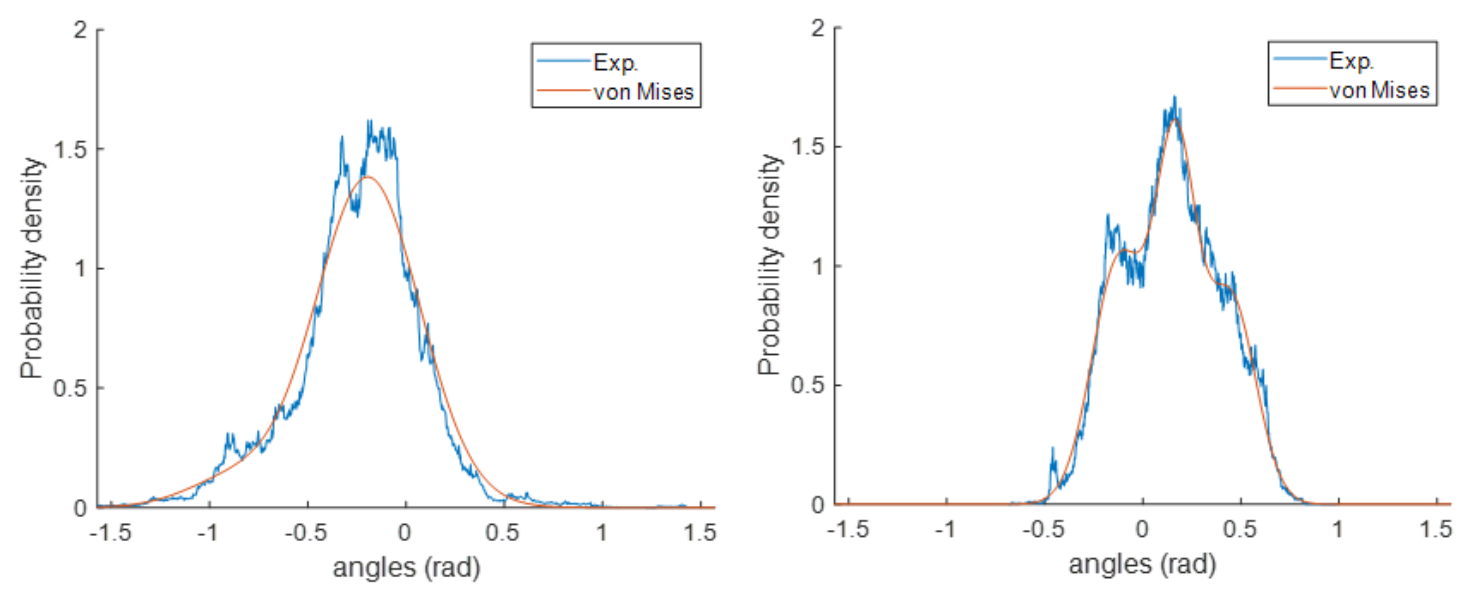

Figure 29: Sample 10, Entire scan analysis - fibre orientations with von Mises fittings (left: no strain, right: $50 \%$ strain) 


\begin{tabular}{|ccc|}
\hline C. P. (\%) & $\boldsymbol{\mu}$ (rad) & K \\
\hline 45.9 & 0.65 & 11.8 \\
\hline 38.4 & -0.29 & 6.8 \\
\hline 15.1 & -0.16 & 360.5 \\
\hline 0.6 & -1.45 & 236.0 \\
\hline
\end{tabular}

\begin{tabular}{|ccc|}
\hline C. P. (\%) & $\boldsymbol{\mu}$ (rad) & K \\
\hline 60.3 & 0.04 & 26.8 \\
\hline 22.8 & 0.17 & 4.4 \\
\hline 16.9 & 0.56 & 37.6 \\
\hline & & \\
\hline
\end{tabular}
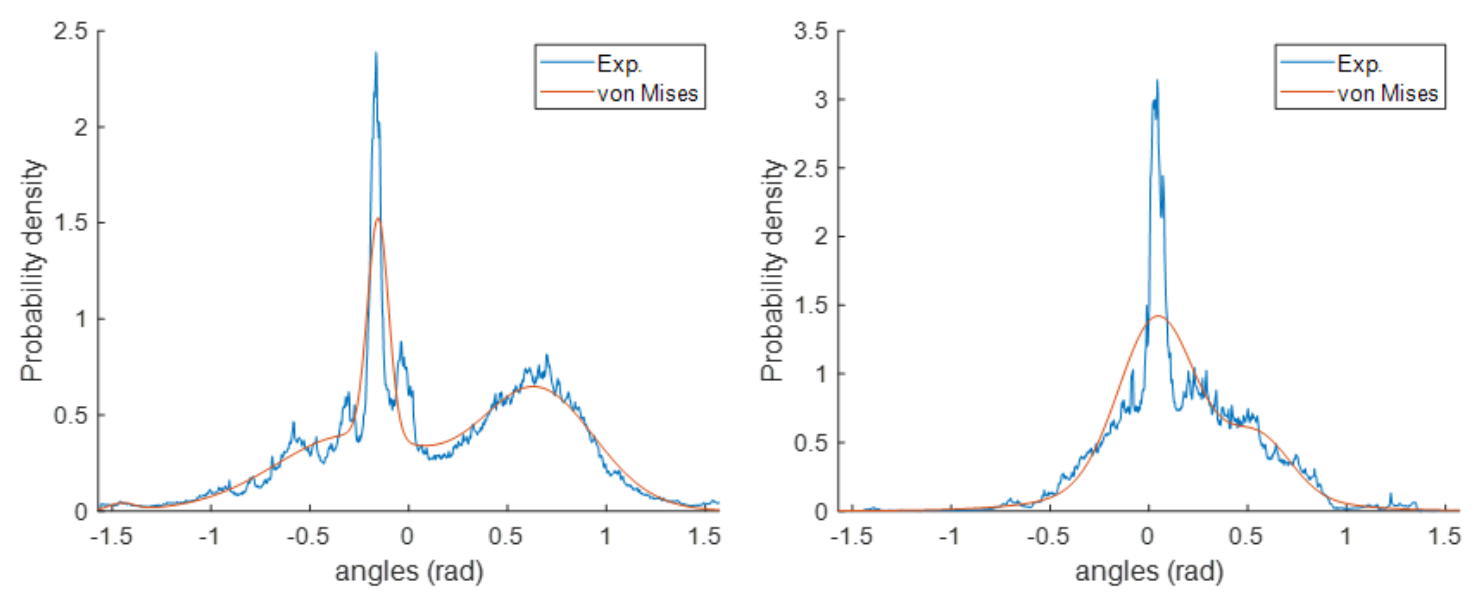

Figure 30: Sample 11, Entire scan analysis - fibre orientations with von Mises fittings (left: no strain, right: $50 \%$ strain) 


\begin{tabular}{|ccc|}
\hline C. P. (\%) & $\boldsymbol{\mu}$ (rad) & K \\
\hline 76.3 & -0.48 & 30.0 \\
\hline 23.7 & -0.19 & 4.4 \\
\hline
\end{tabular}

\begin{tabular}{|ccc|}
\hline C. P. (\%) & $\boldsymbol{\mu}$ (rad) & K \\
\hline 62.4 & -0.06 & 33.5 \\
\hline 24.3 & 0.05 & 221.8 \\
\hline 13.3 & 0.24 & 7.5 \\
\hline
\end{tabular}
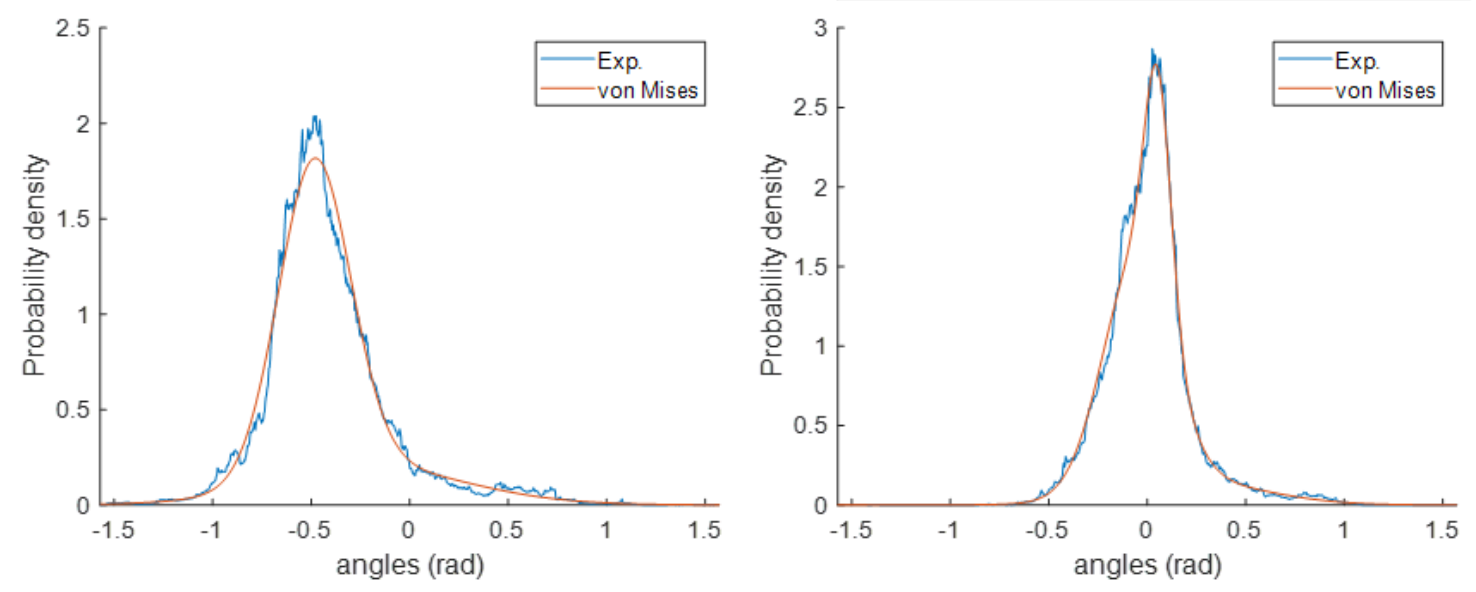

Figure 31: Sample 12, Entire scan analysis - fibre orientations with von Mises fittings (left: no strain, right: $50 \%$ strain)

\begin{tabular}{|ccc|}
\hline C. P. (\%) & $\boldsymbol{\mu}$ (rad) & K \\
\hline 41.9 & 0.30 & 8.2 \\
\hline 29.7 & 0.13 & 60.2 \\
\hline 28.4 & -0.16 & 67.1 \\
\hline
\end{tabular}

\begin{tabular}{|ccc|}
\hline C. P. (\%) & $\boldsymbol{\mu}$ (rad) & K \\
\hline 56.8 & 0.15 & 19.9 \\
\hline 41.7 & 0.12 & 85.3 \\
\hline 1.5 & -0.03 & 2.3 \\
\hline
\end{tabular}
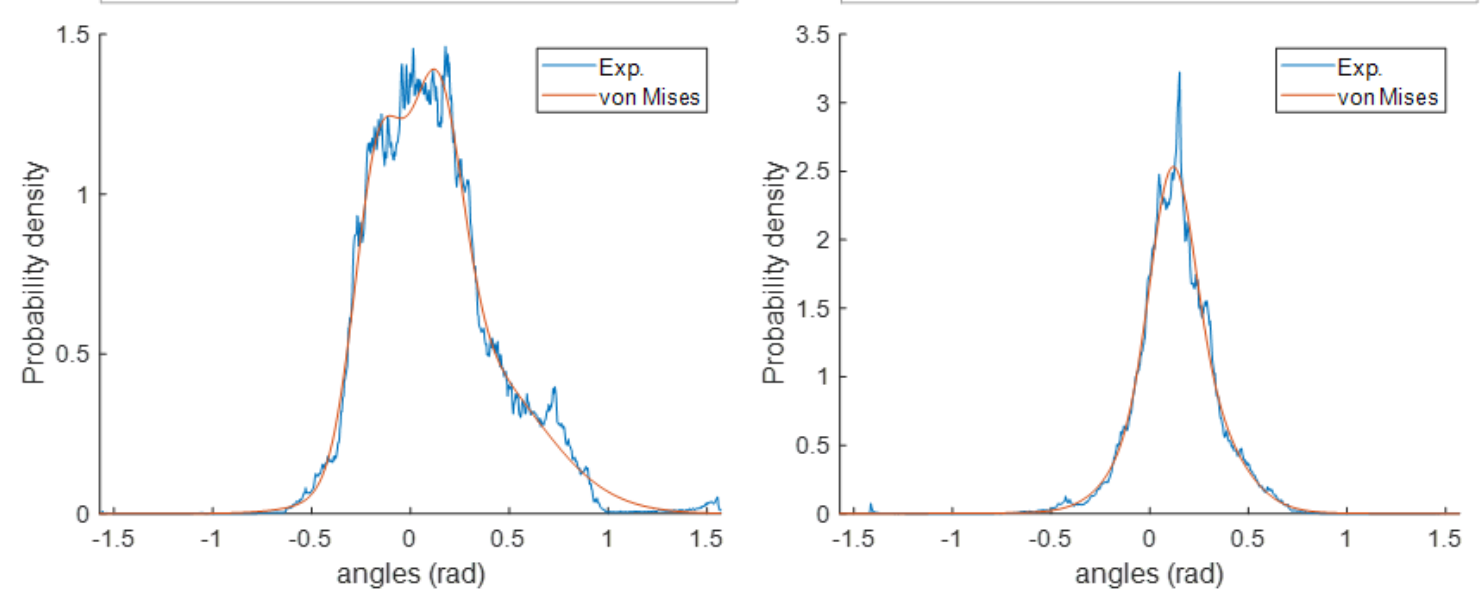

Figure 32: Sample 13, Entire scan analysis - fibre orientations with von Mises fittings (left: no strain, right: $50 \%$ strain) 


\begin{tabular}{|ccc|}
\hline Comp. Prop. (\%) & $\boldsymbol{\mu}$ (rad) & $\boldsymbol{~}$ \\
\hline 50.7 & -0.92 & 14.5 \\
\hline 32.5 & 0.22 & 4.1 \\
\hline 16.8 & -0.23 & 28.9 \\
\hline
\end{tabular}

\begin{tabular}{|ccc|}
\hline Comp. Prop. (\%) & $\boldsymbol{\mu}$ (rad) & K \\
\hline 60.4 & -0.07 & 18.8 \\
\hline 38.3 & -0.51 & 12.8 \\
\hline 1.3 & 0.93 & 5.5 \\
\hline
\end{tabular}
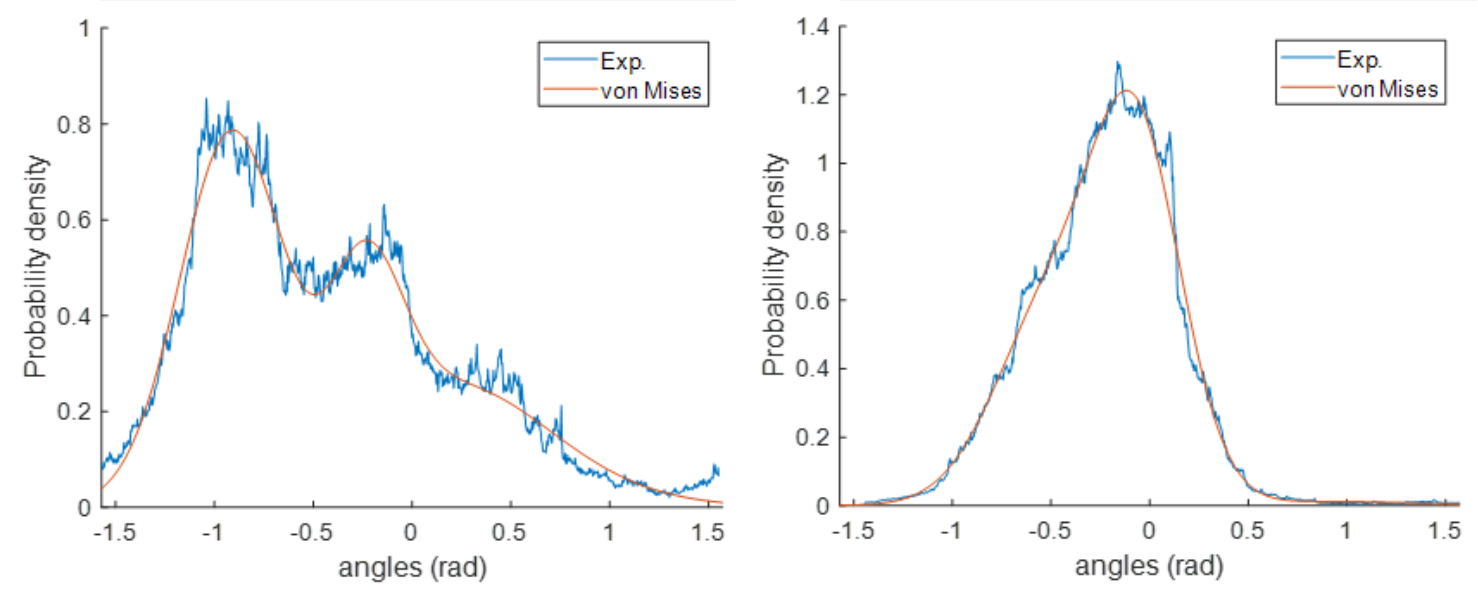

Figure 33: Sample 14, Entire scan analysis - fibre orientations with von Mises fittings (left: no strain, right: $50 \%$ strain)

\begin{tabular}{|ccc|}
\hline C. P. (\%) & $\boldsymbol{\mu}$ (rad) & K \\
\hline 59.2 & -0.26 & 35.2 \\
\hline 40.8 & 0.06 & 4.8 \\
\hline & & \\
\hline
\end{tabular}

\begin{tabular}{|ccc|}
\hline C. P. (\%) & $\boldsymbol{\mu}$ (rad) & K \\
\hline 97.3 & -0.37 & 27.2 \\
\hline 2.7 & -0.36 & 2.8 \\
\hline & & \\
\hline
\end{tabular}
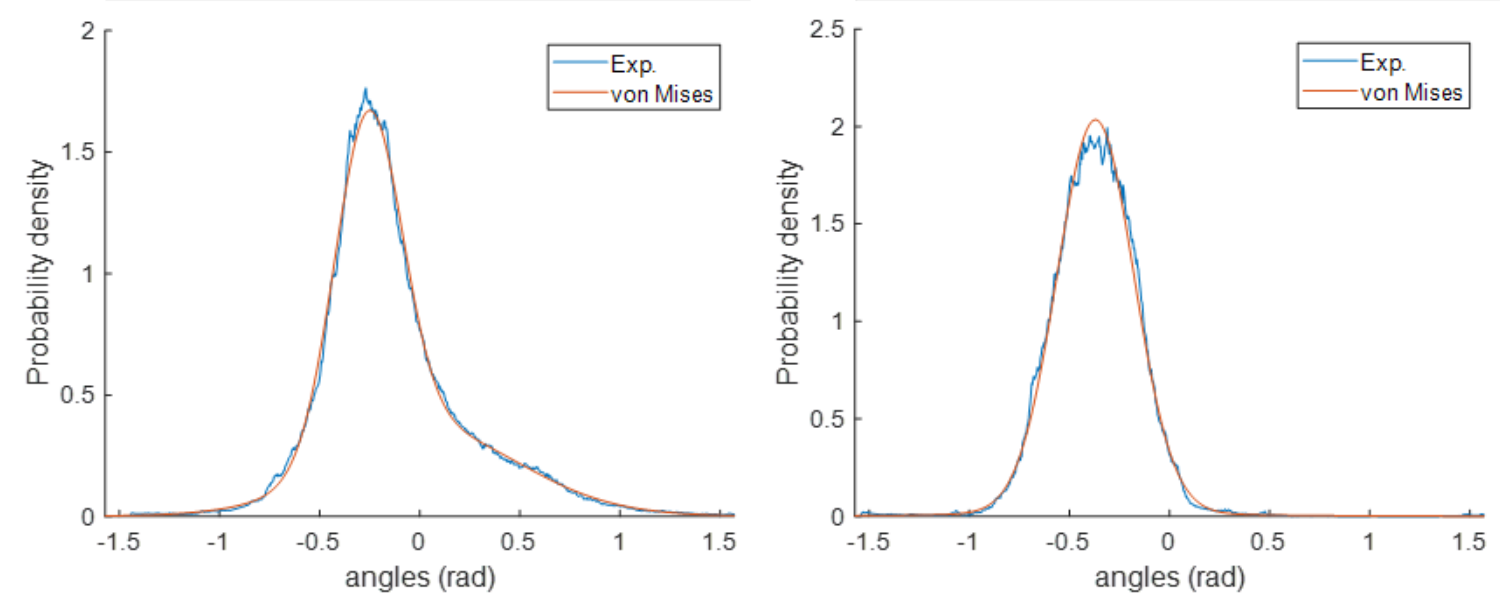

Figure 34: Sample 15, Entire scan analysis - fibre orientations with von Mises fittings (left: no strain, right: $50 \%$ strain) 


\begin{tabular}{|ccc|}
\hline C. $\mathbf{P .}$ (\%) & $\boldsymbol{\mu}$ (rad) & K \\
\hline 50.6 & 0.29 & 32.2 \\
\hline 38.7 & -0.22 & 29.2 \\
\hline 10.7 & 0.09 & 3.9 \\
\hline
\end{tabular}

\begin{tabular}{|ccc|}
\hline C. P. (\%) & $\boldsymbol{\mu}$ (rad) & K \\
\hline 70.9 & 0.11 & 50.2 \\
\hline 29.1 & 0.21 & 7.2 \\
\hline & & \\
\hline
\end{tabular}
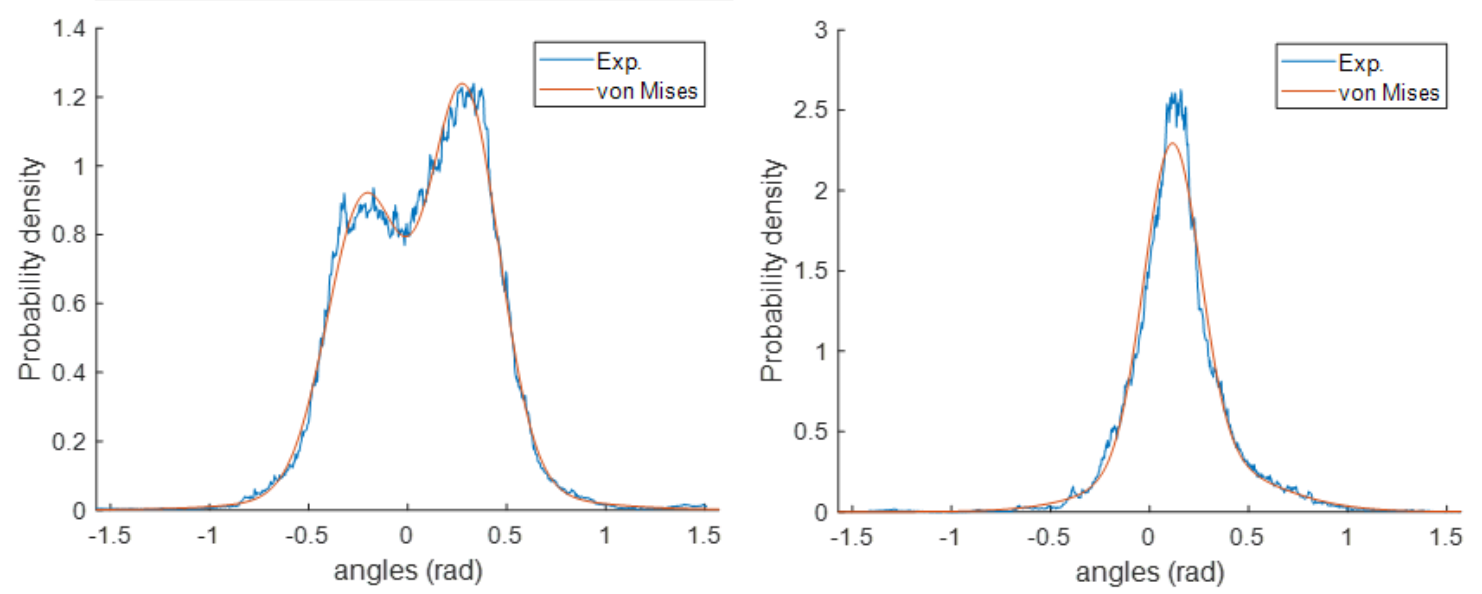

Figure 35: Sample 16, Entire scan analysis - fibre orientations with von Mises fittings (left: no strain, right: $50 \%$ strain)

\begin{tabular}{|ccc|}
\hline C. P. (\%) & $\boldsymbol{\mu}$ (rad) & K \\
\hline 41.4 & -0.49 & 22.7 \\
\hline 30.7 & -0.02 & 49.6 \\
\hline 27.9 & 0.41 & 7.6 \\
\hline & & \\
\hline
\end{tabular}

\begin{tabular}{|ccc|}
\hline C. P. (\%) & $\boldsymbol{\mu}$ (rad) & K \\
\hline 57.9 & -0.14 & 24.1 \\
\hline 20.0 & 0.02 & 5.1 \\
\hline 14.9 & -0.03 & 357.7 \\
\hline 7.2 & 0.41 & 133.1 \\
\hline
\end{tabular}
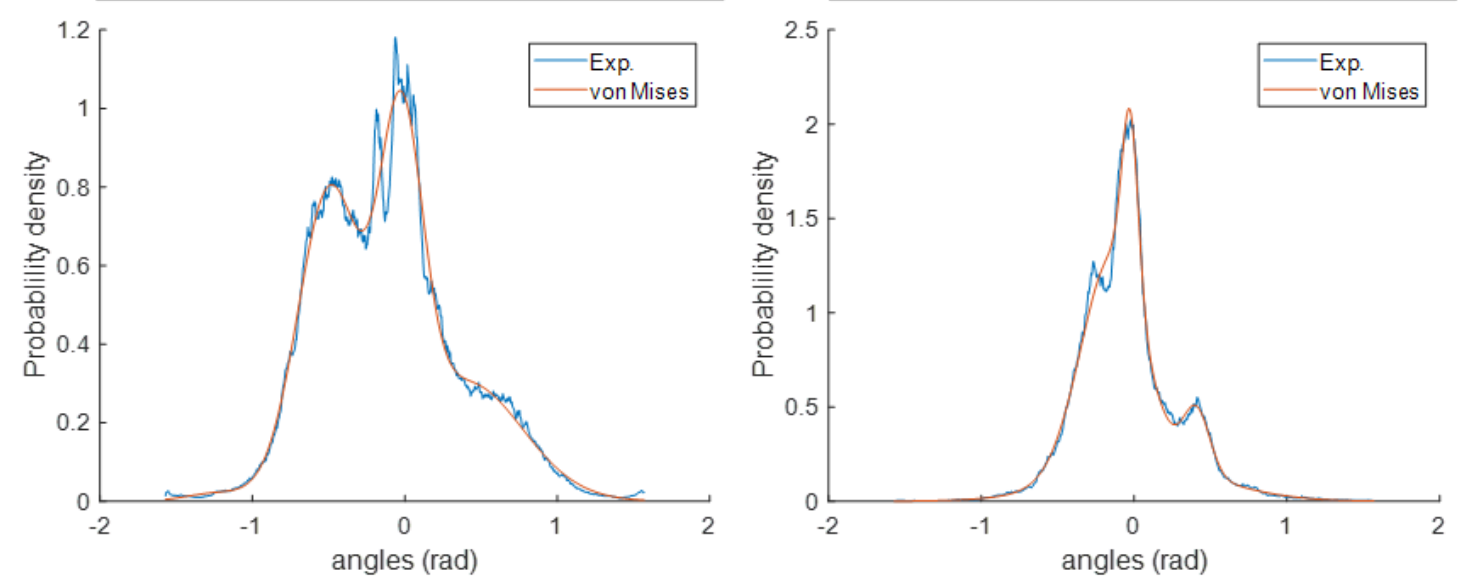

Figure 36: Frontal lobe group, Entire scan analysis - fibre orientations with von Mises fittings for the frontal lobe (left: no strain, right: $50 \%$ strain) 


\begin{tabular}{|ccc|}
\hline C. P. (\%) & $\boldsymbol{\mu}$ (rad) & K \\
\hline 67.1 & -0.02 & 28.8 \\
\hline 17.7 & -0.84 & 12.0 \\
\hline 15.1 & 0.51 & 21.7 \\
\hline
\end{tabular}

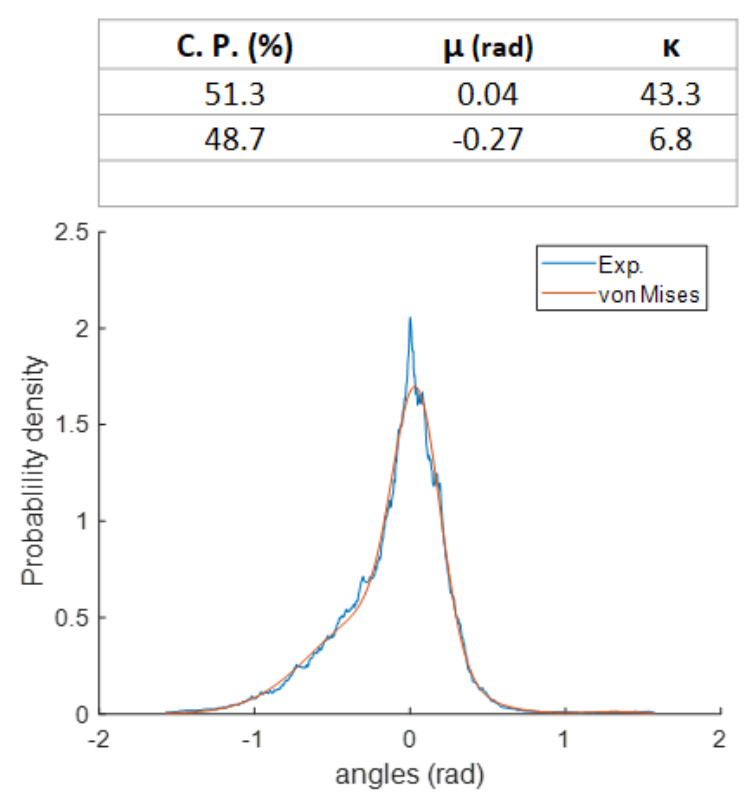

Figure 37: Parietal lobe group, Entire scan analysis - fibre orientations with von Mises fittings for the parietal lobe (left: no strain, right: 50\% strain)

\begin{tabular}{|ccc|}
\hline C. P. (\%) & $\boldsymbol{\mu}$ (rad) & K \\
\hline 77.0 & -0.30 & 18.2 \\
\hline 23.1 & 0.31 & 21.4 \\
\hline & & \\
\hline
\end{tabular}

\begin{tabular}{|ccc|}
\hline C. P. (\%) & $\boldsymbol{\mu}$ (rad) & K \\
\hline 82.5 & -0.28 & 17.3 \\
\hline 12.9 & -0.06 & 126.3 \\
\hline 4.7 & -0.1 & 3.7 \\
\hline
\end{tabular}
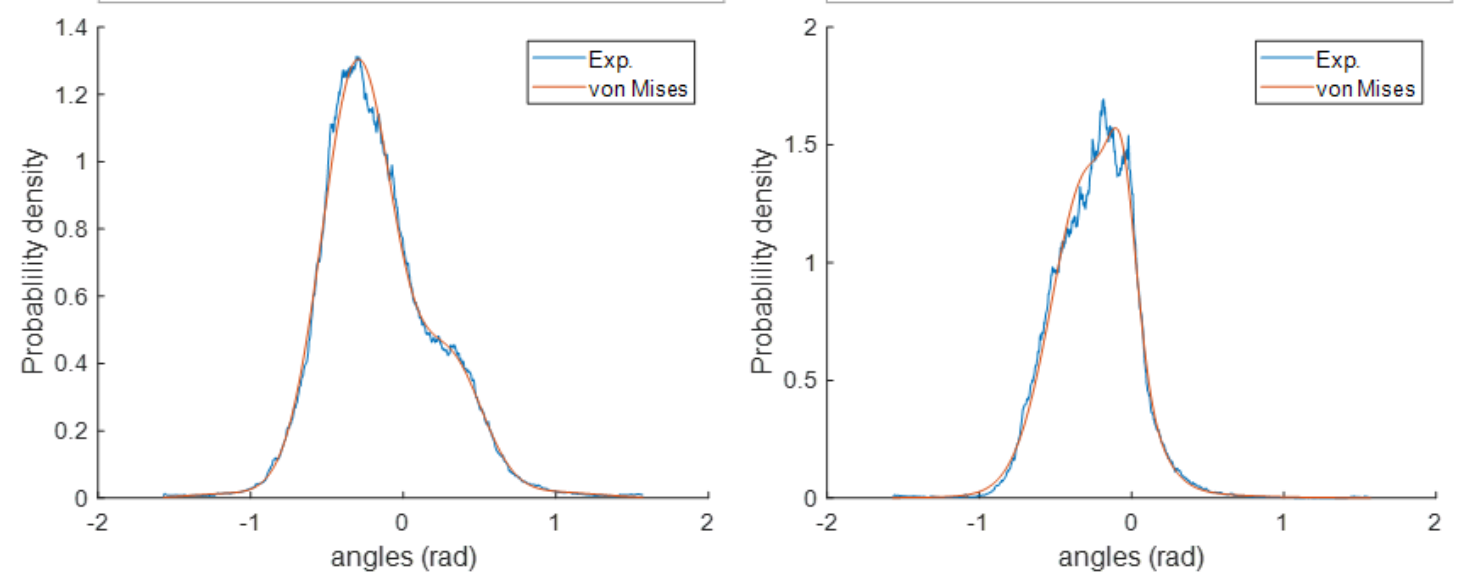

Figure 38: Occipital lobe group, Entire scan analysis - fibre orientations with von Mises fittings for the occipital lobe (left: no strain, right: 50\% strain) 


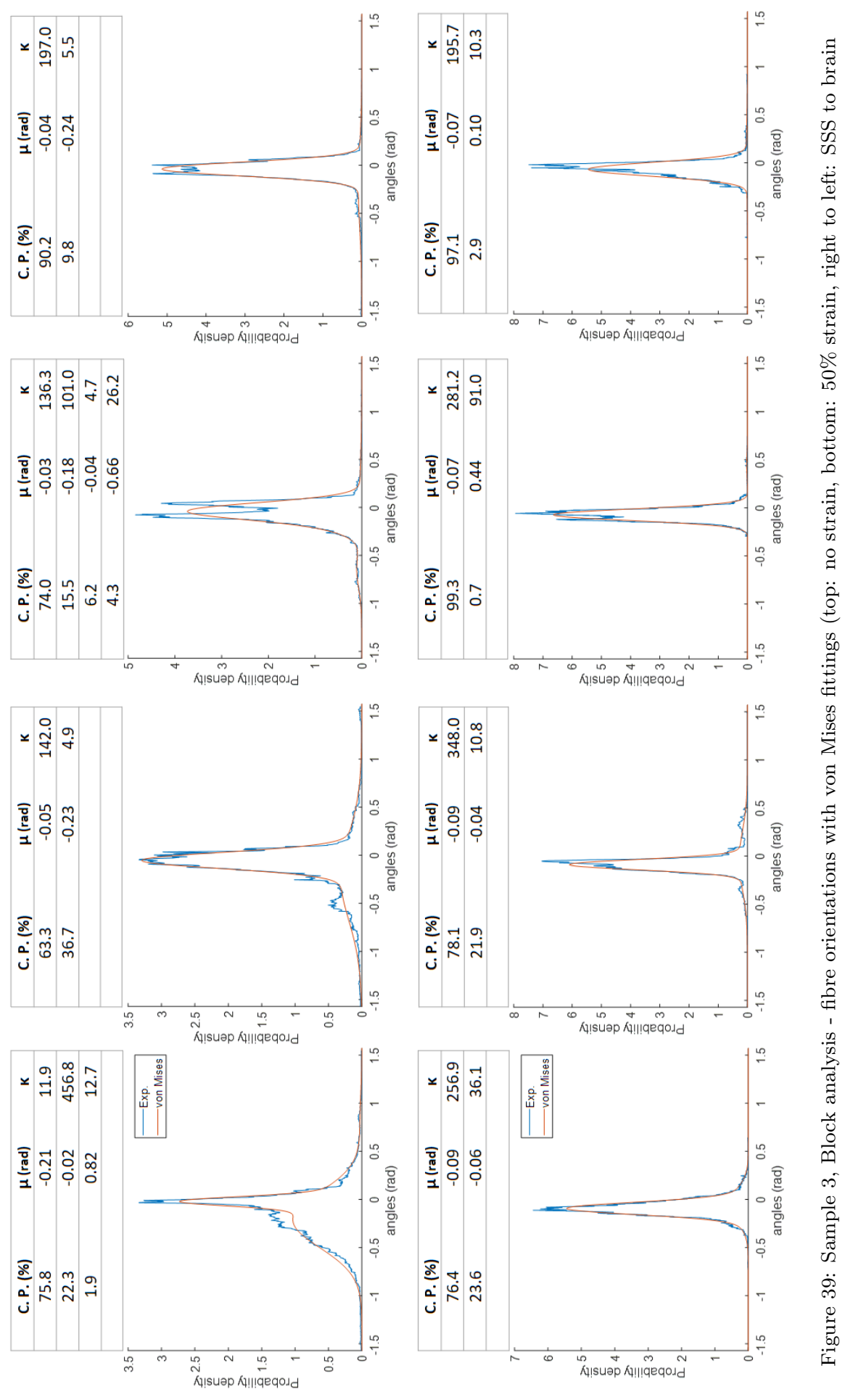



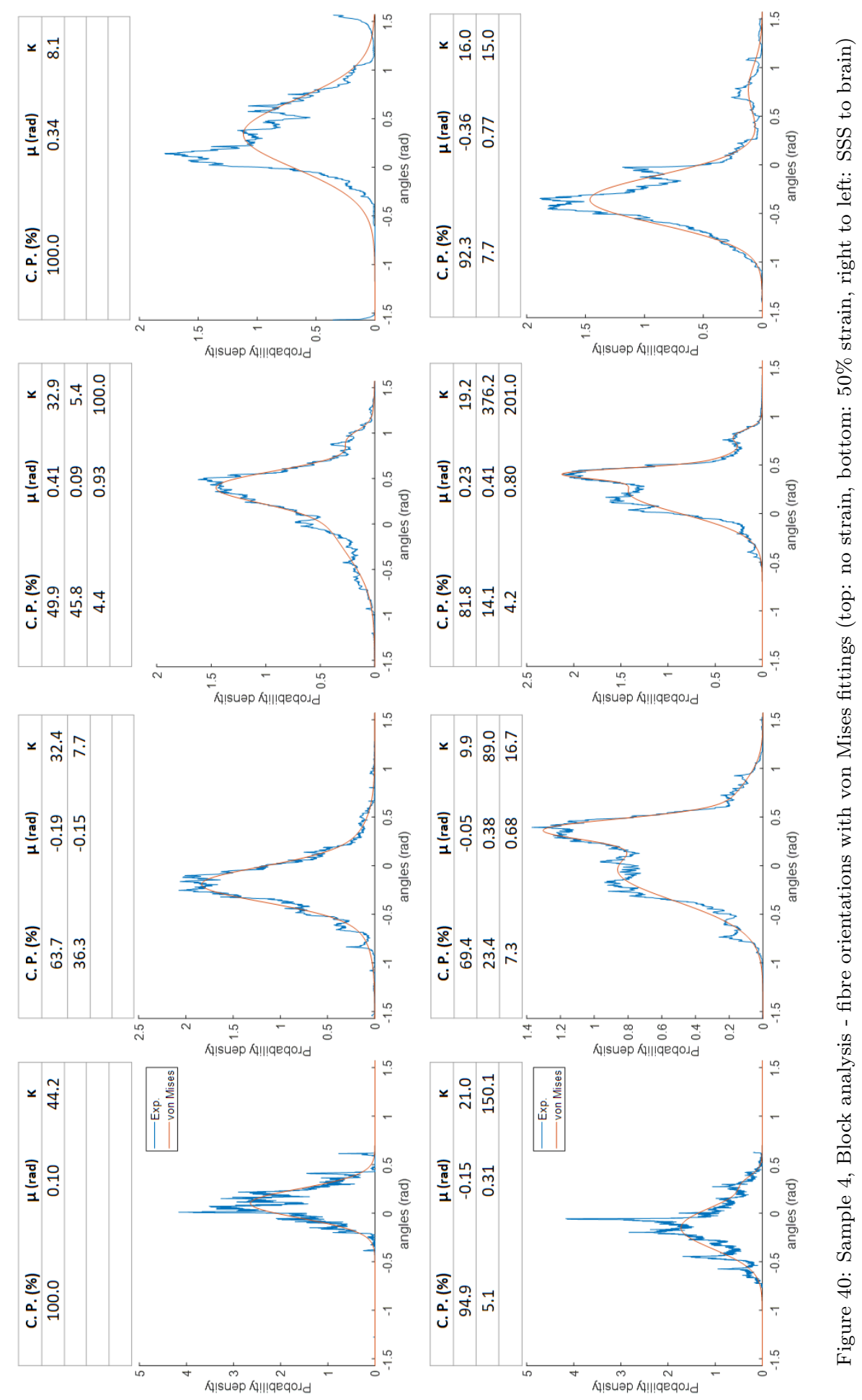

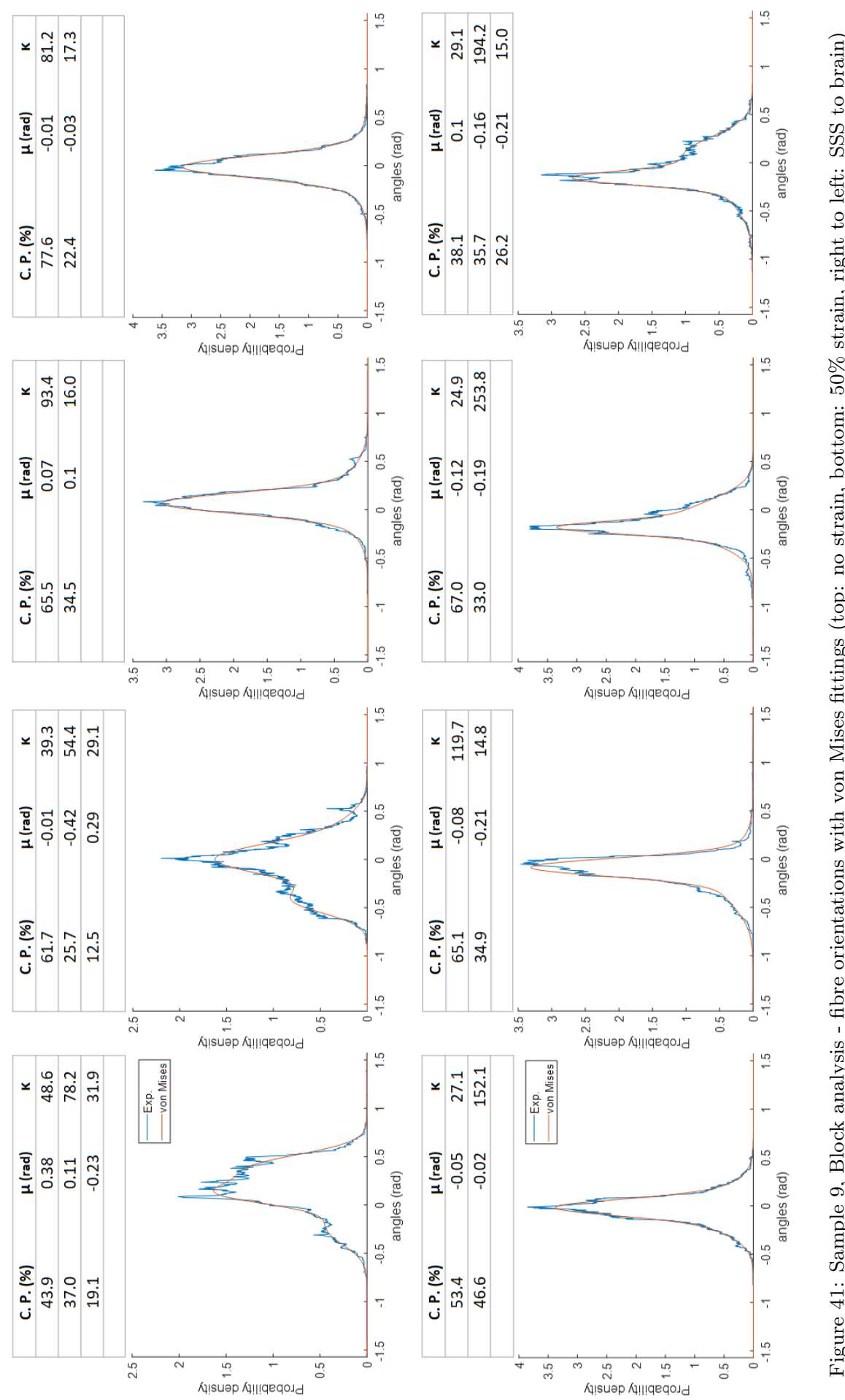

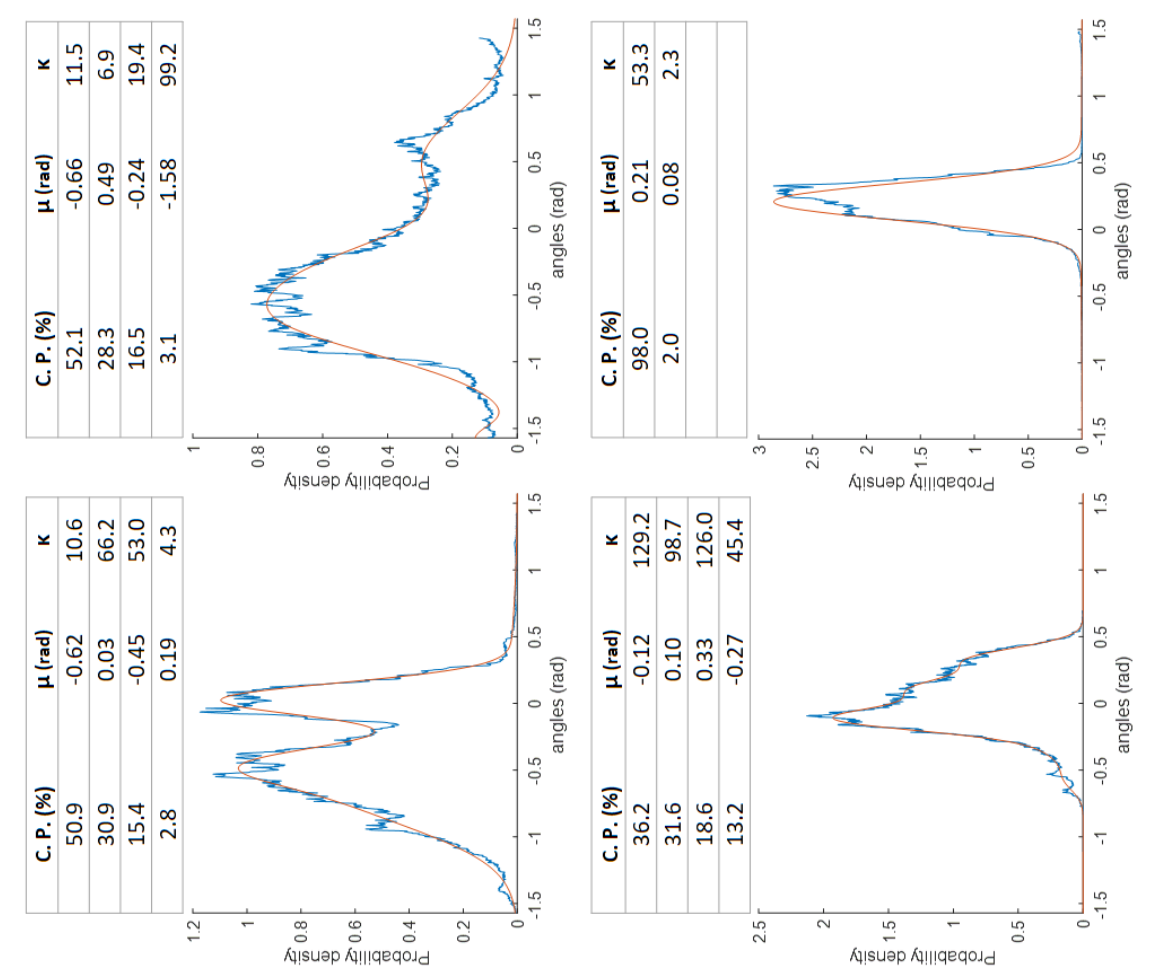

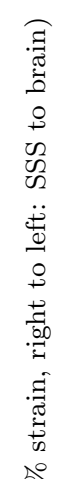
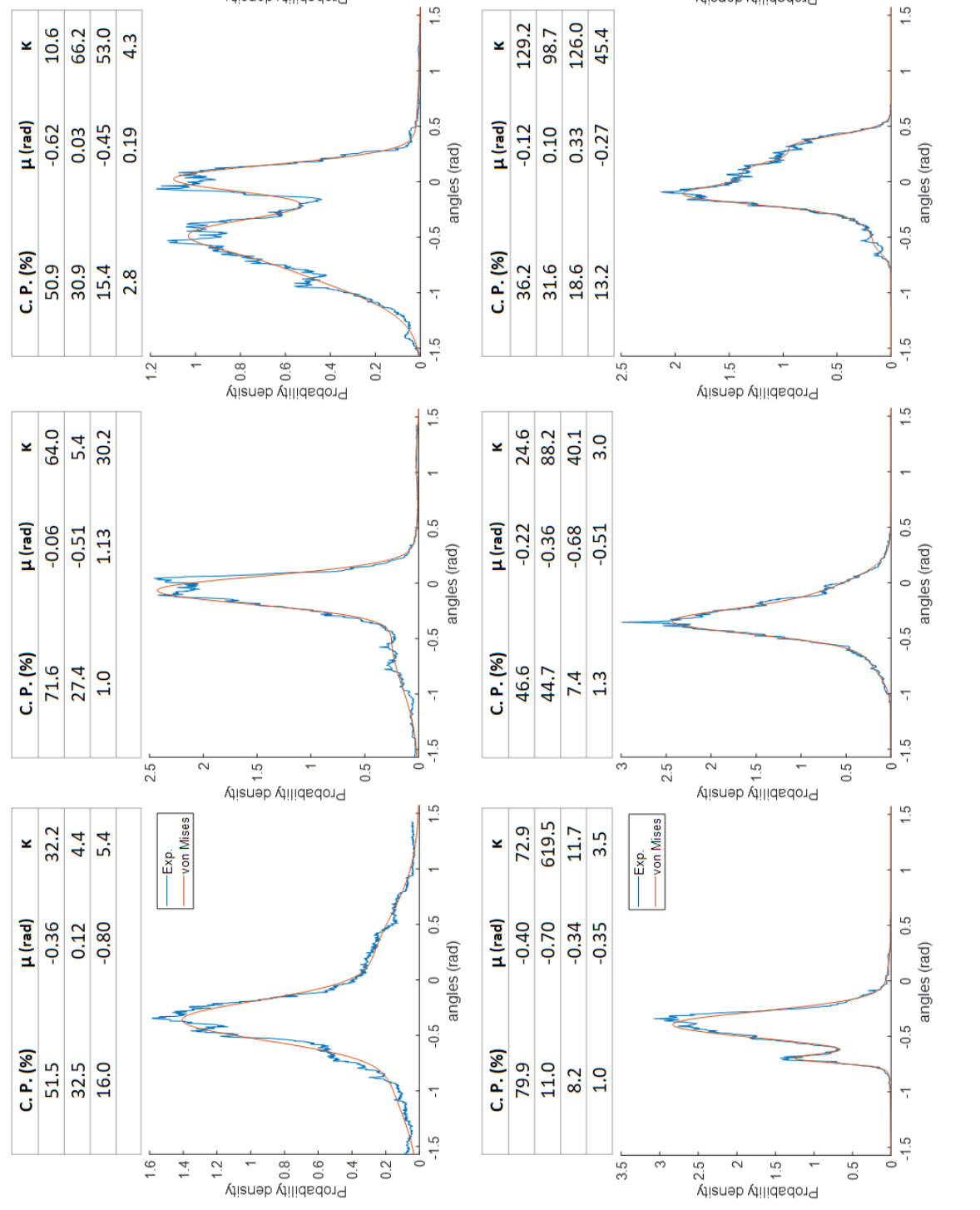

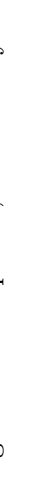



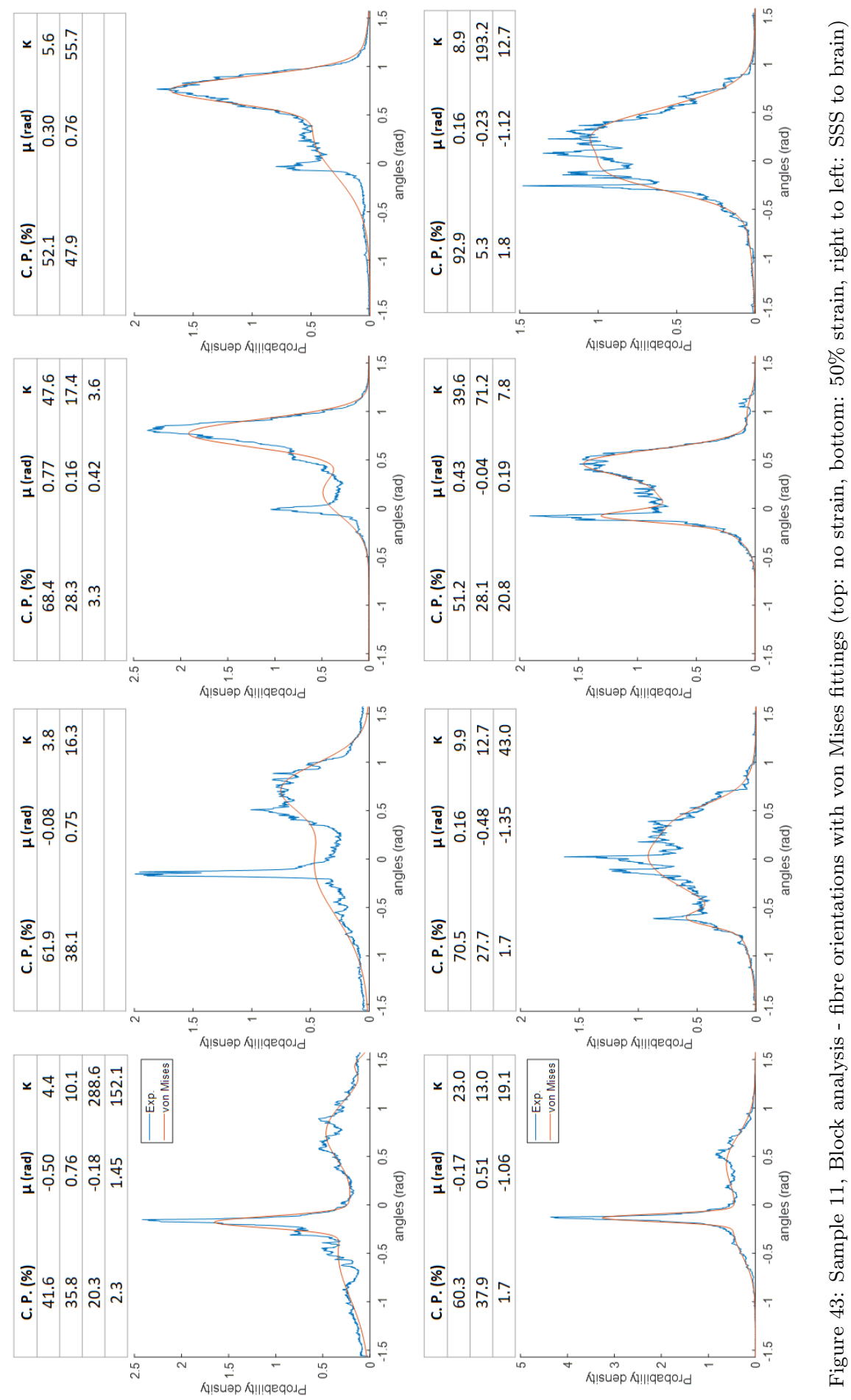

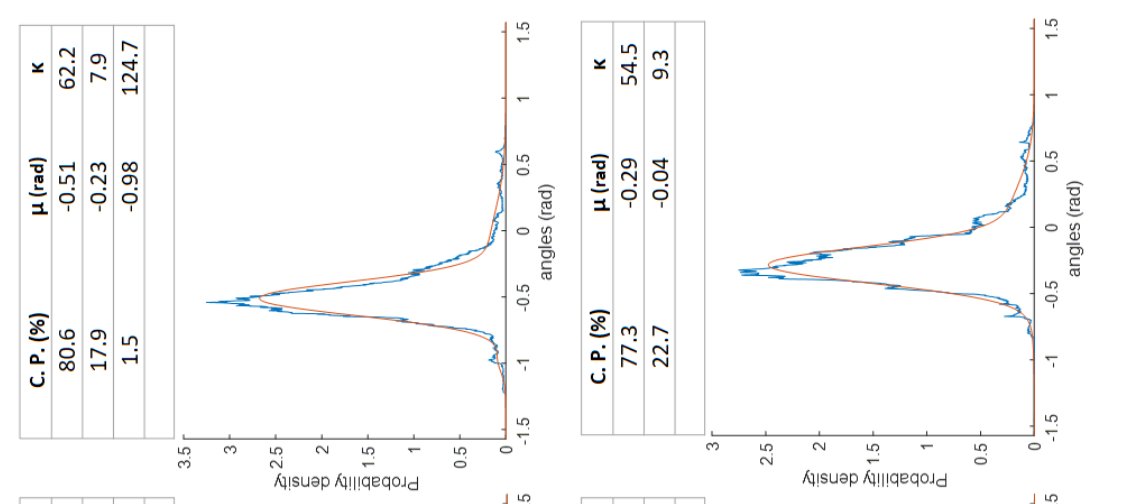

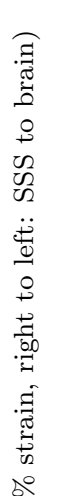
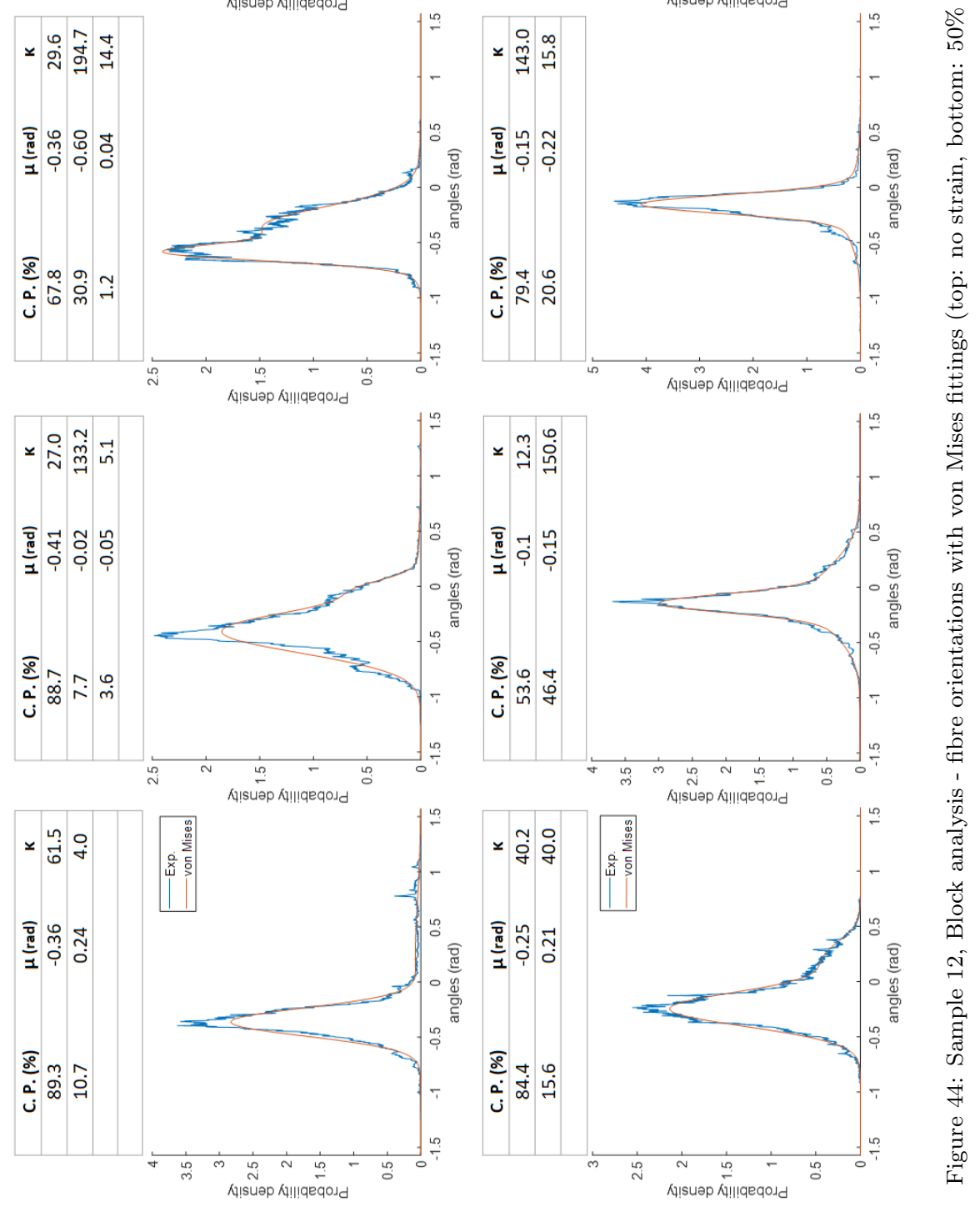

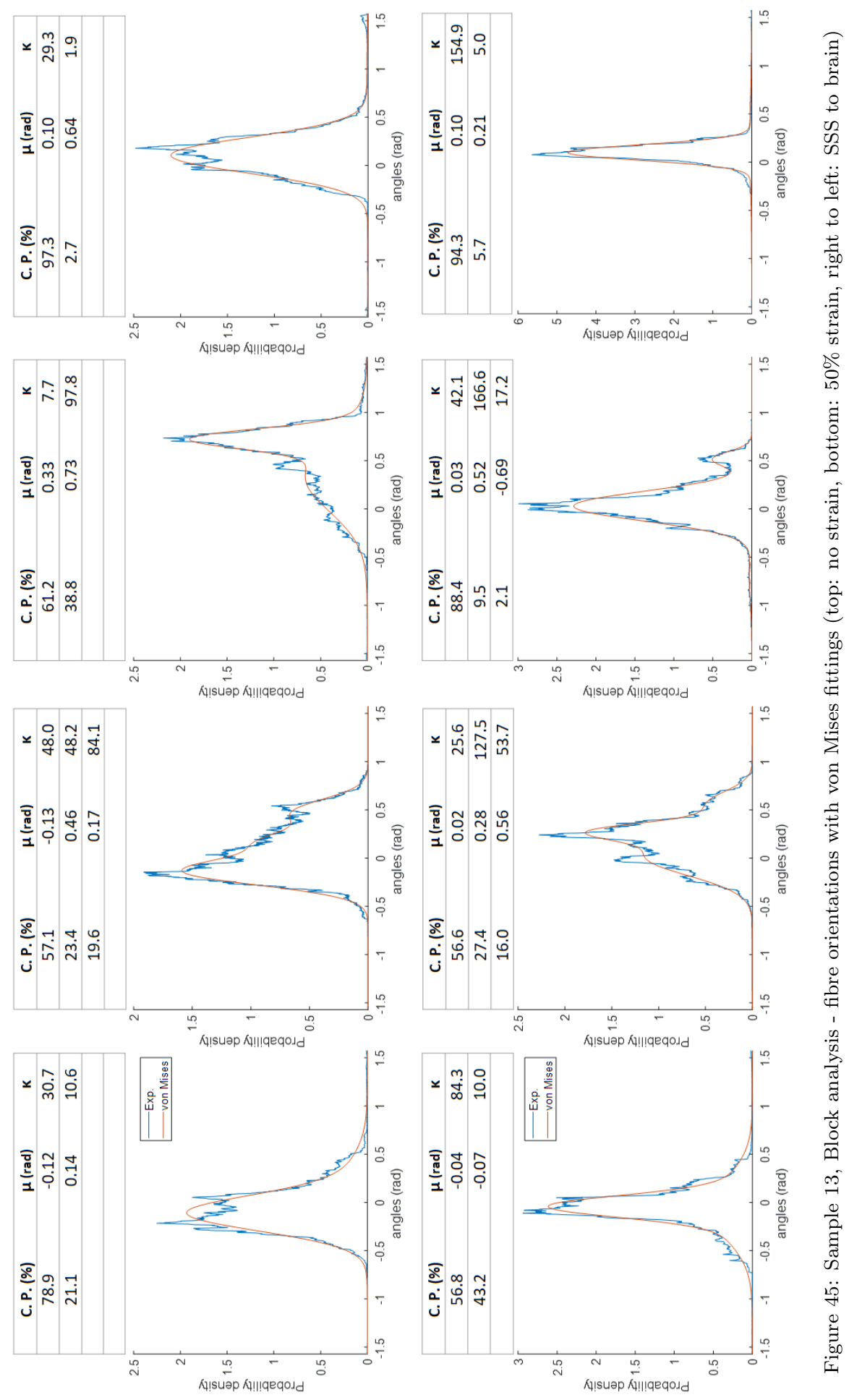

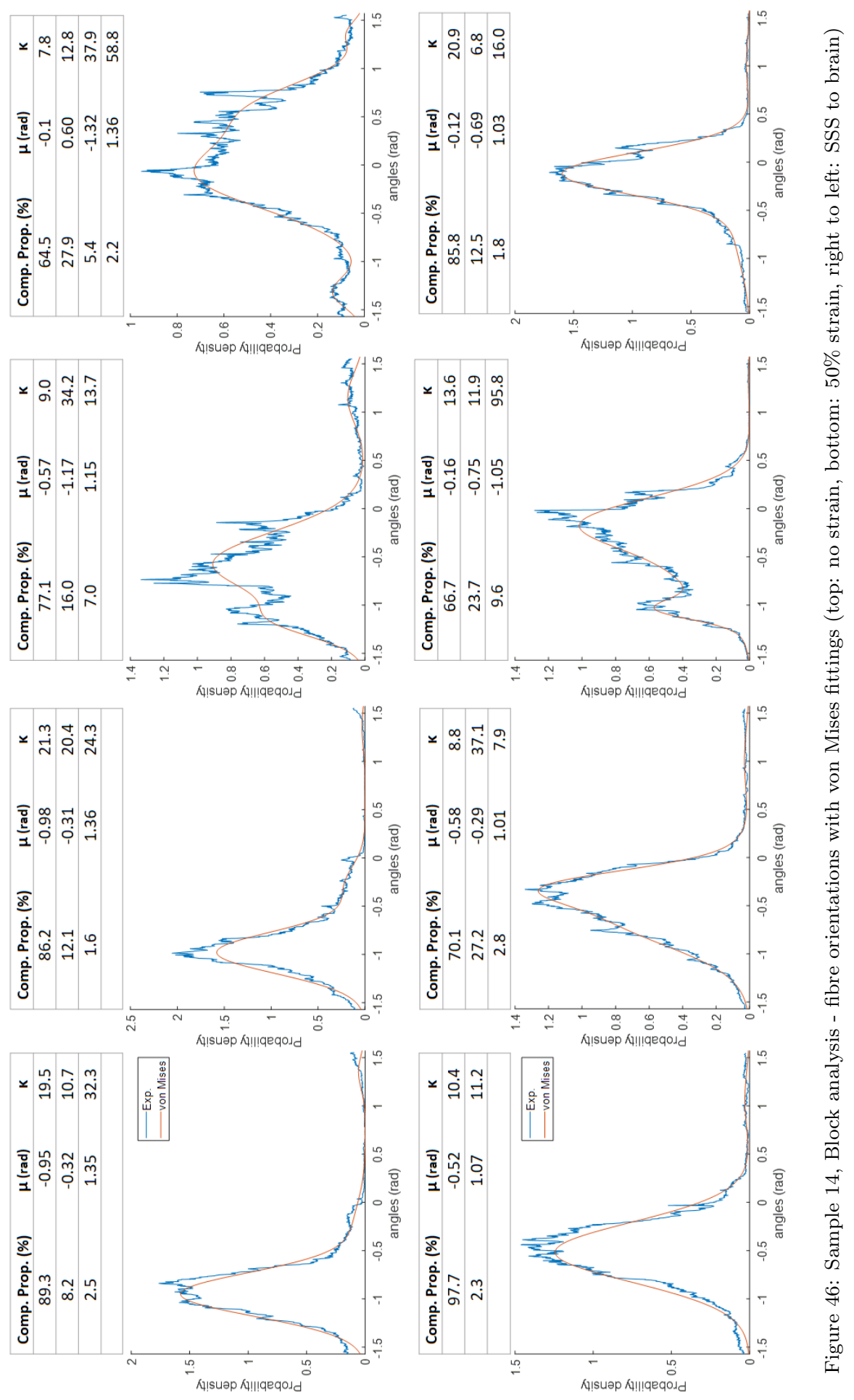


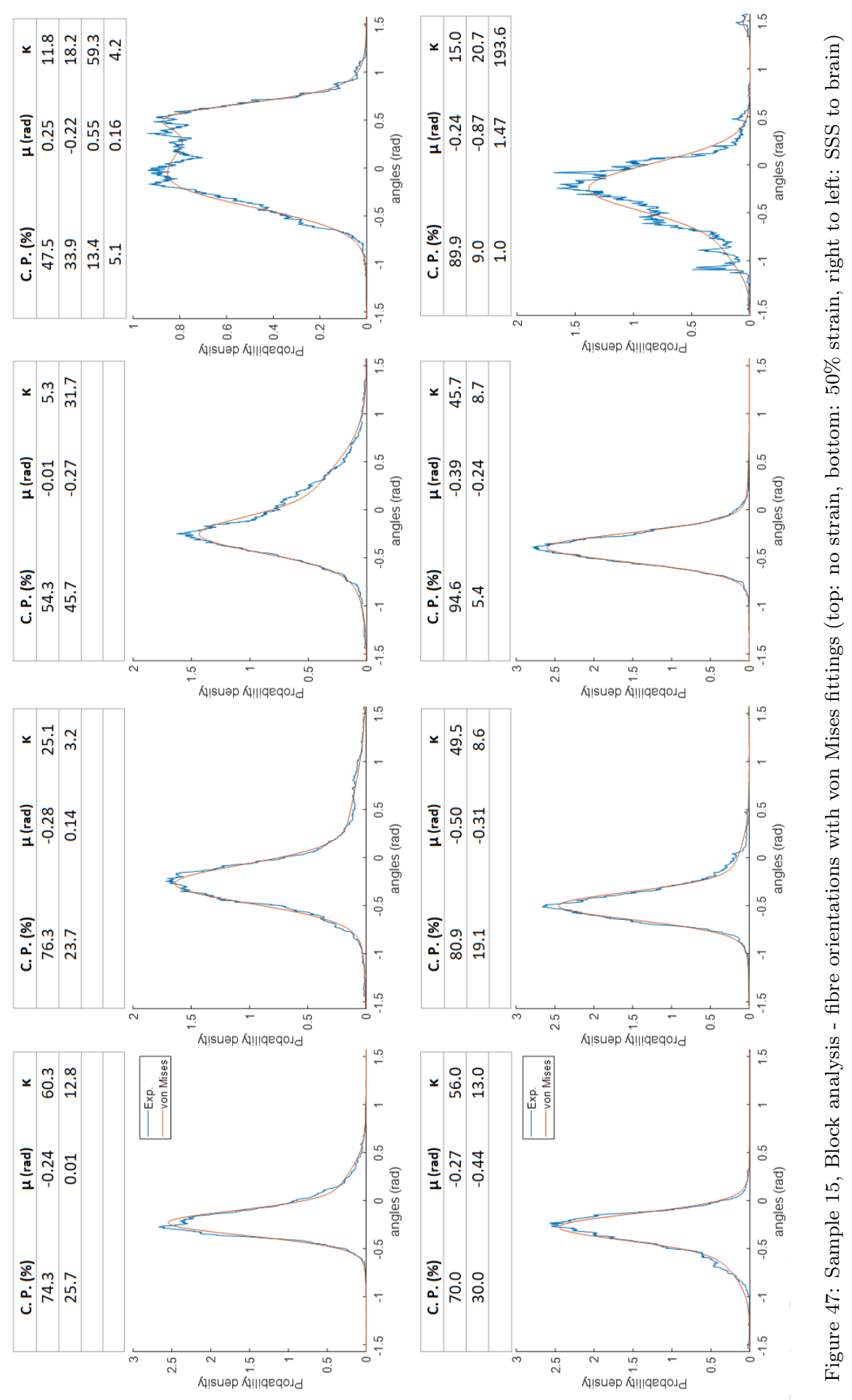



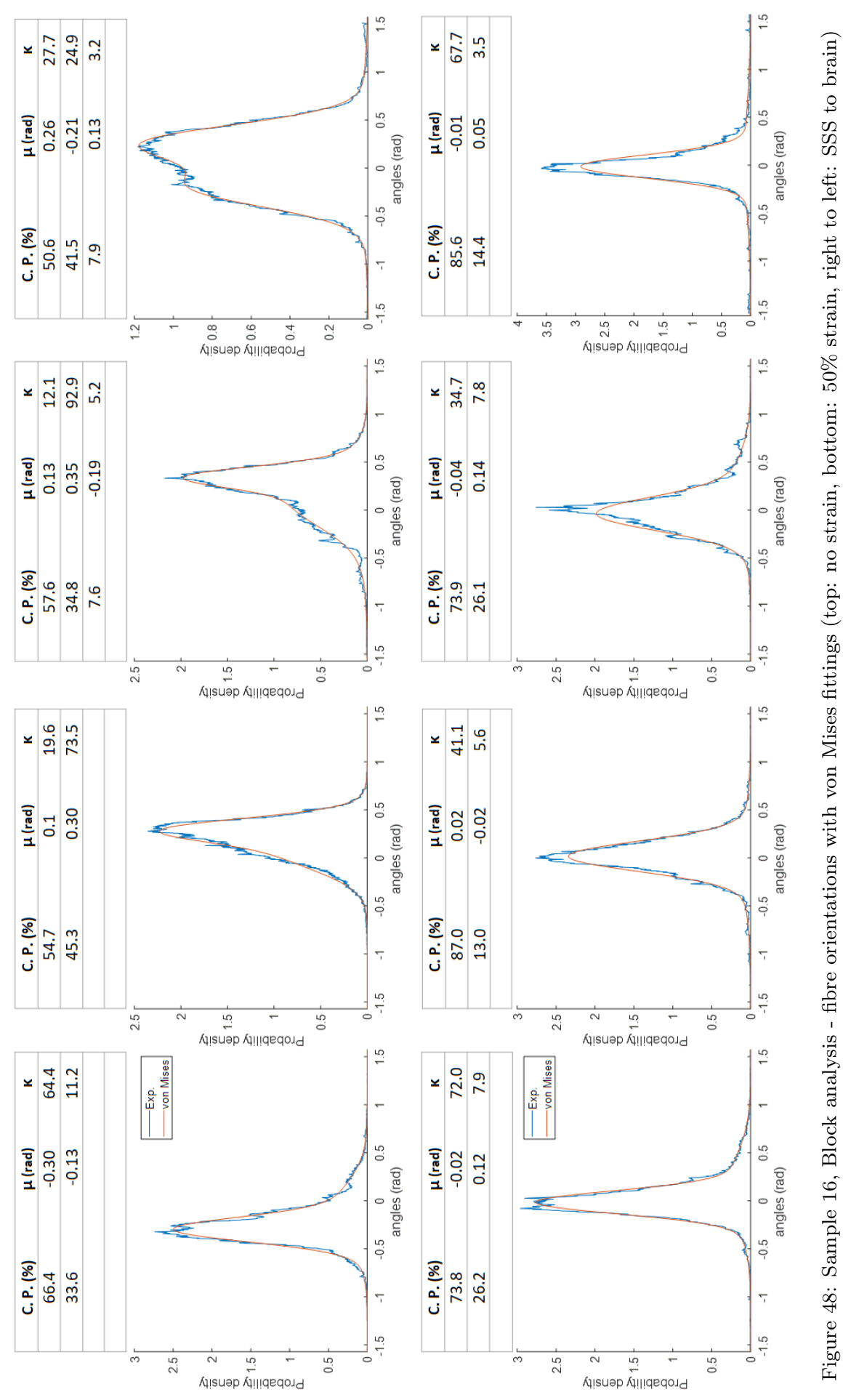


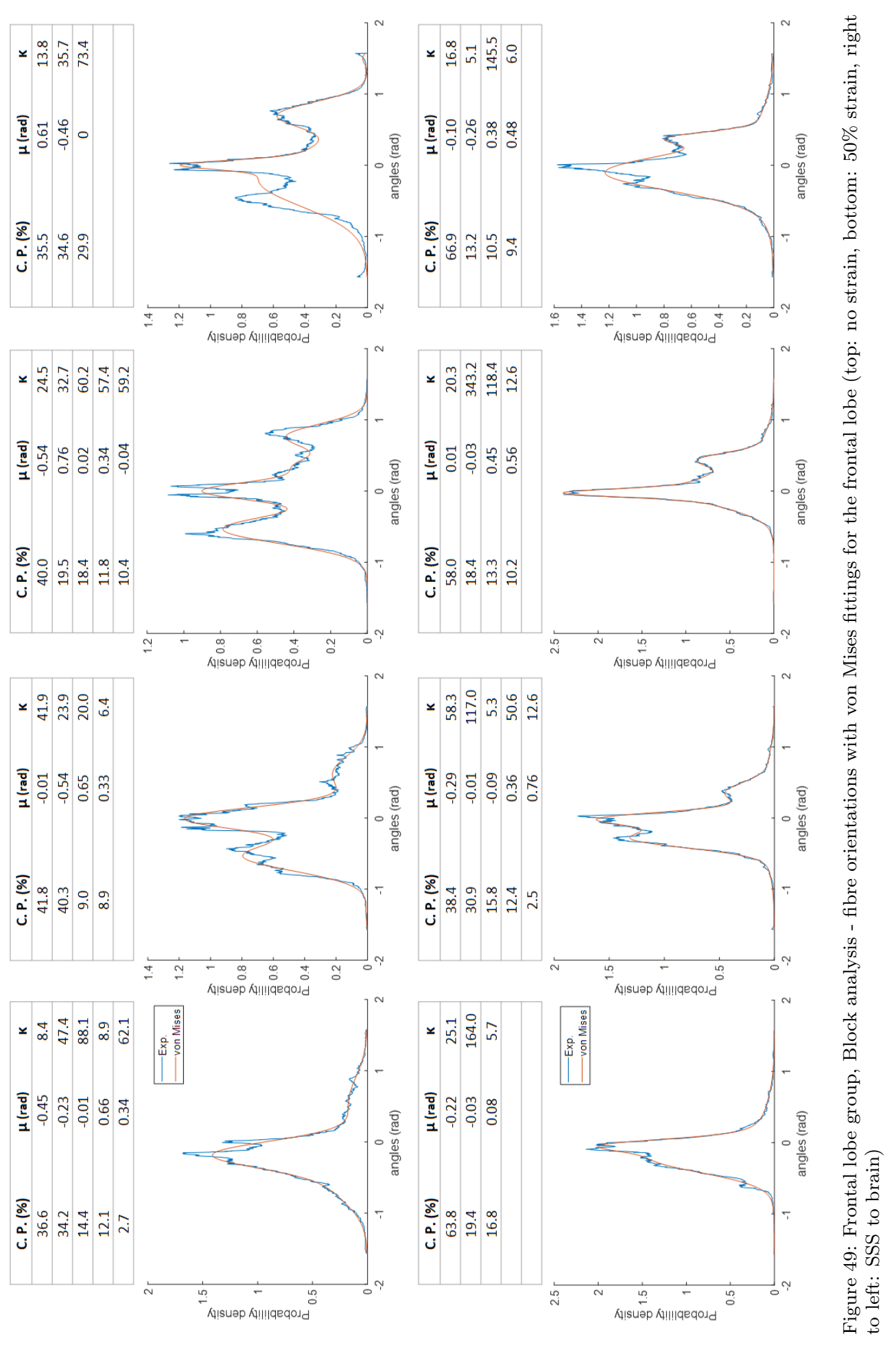



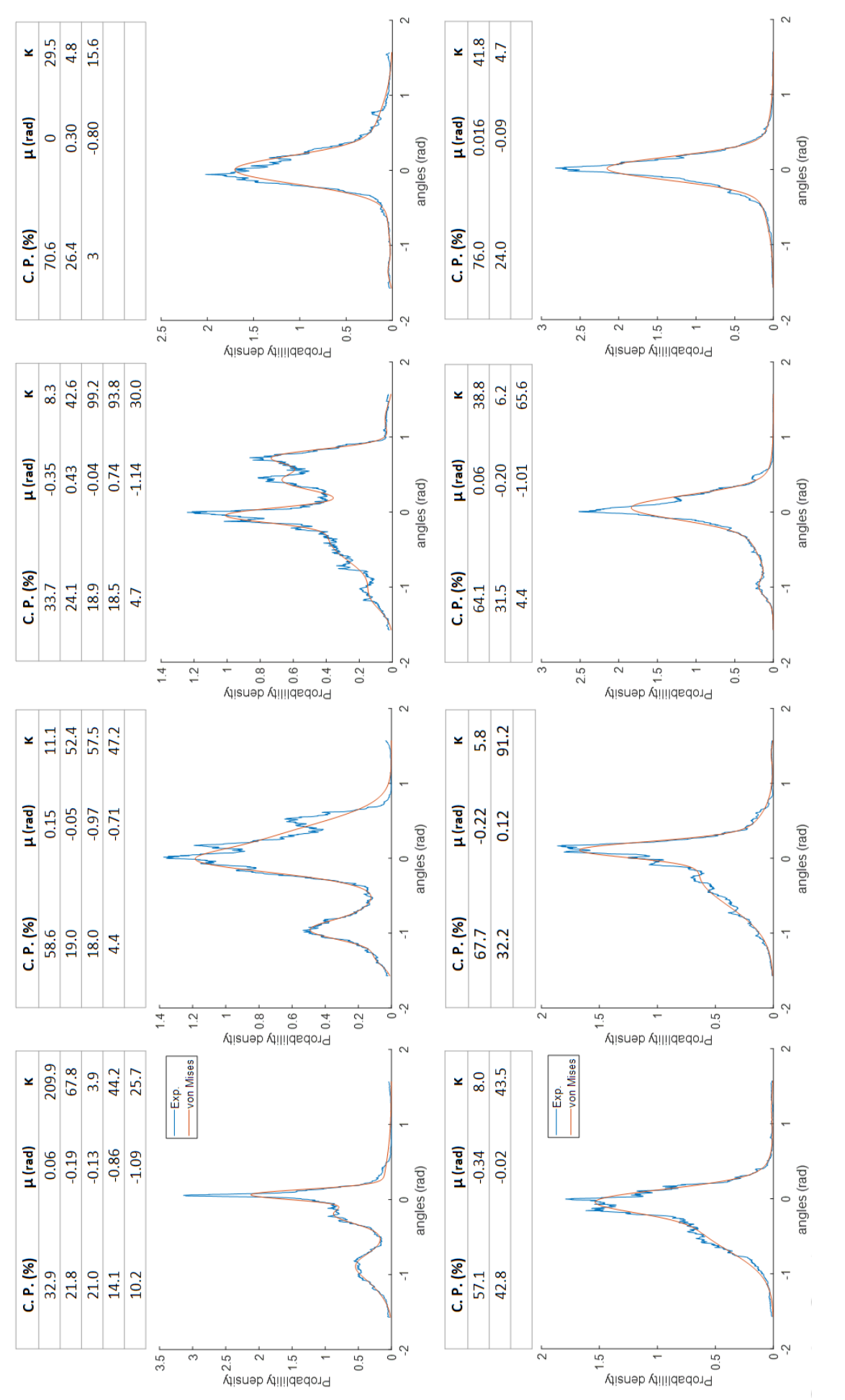

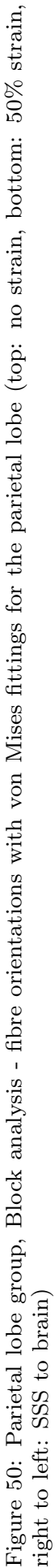



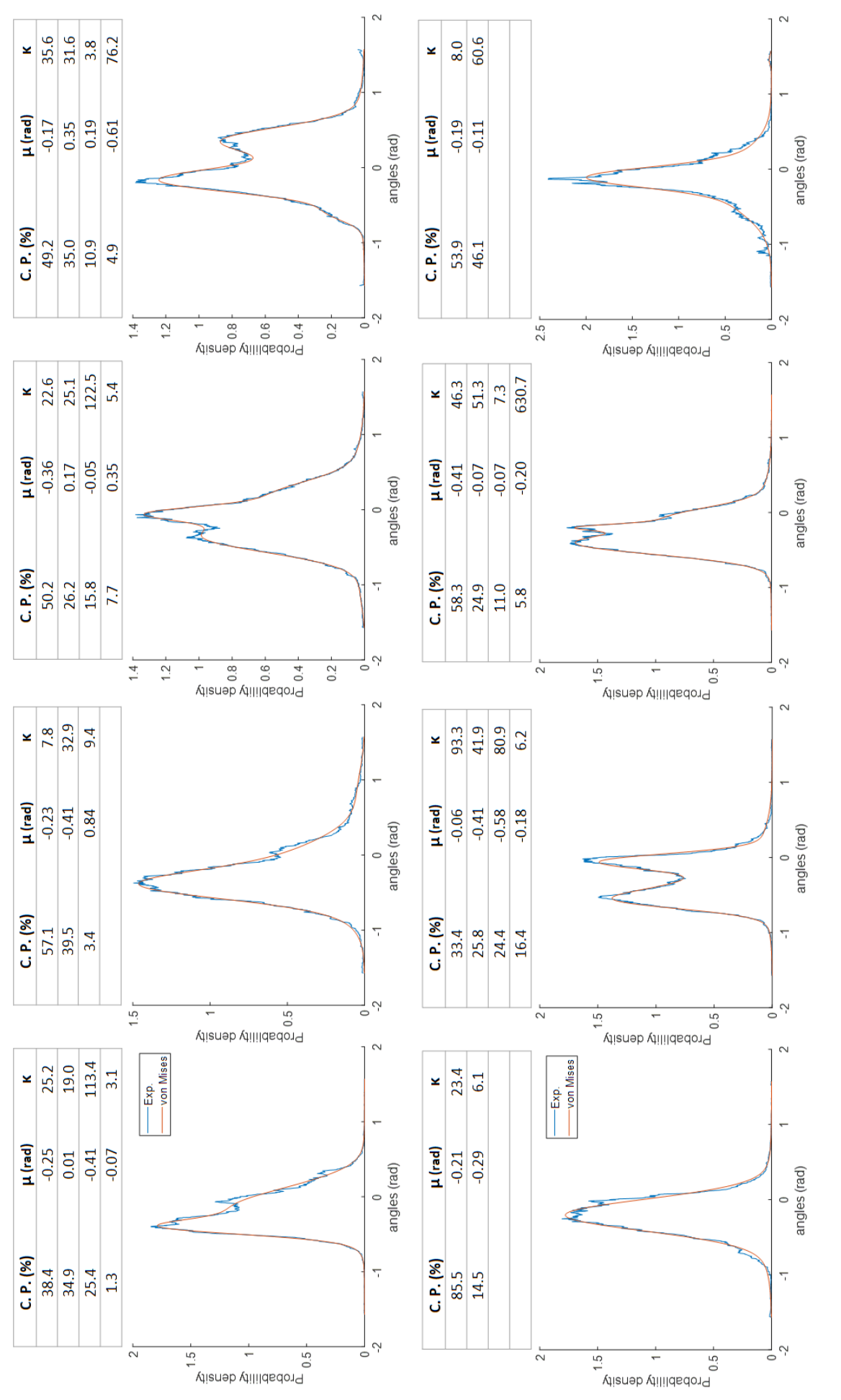

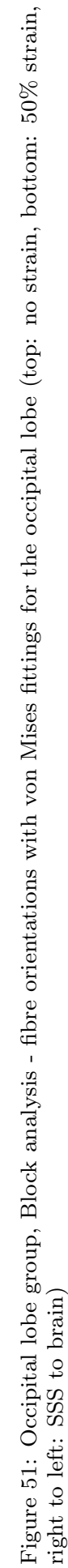

